\title{
2 Beschreibung und quantitative Erfassung der Inschriften
}

\subsection{Inhaltliches Spektrum der Inschriften in Pompeji und Herculaneum}

In Pompeji und Herculaneum gibt es in Stein gemeißelte Inschriften, gemalte Inschriften (Dipinti) und geritzte Inschriften (Graffiti) sowie vereinzelt Mosaikinschriften. Anhand des umfangreichen in diesen beiden Städten erhaltenen Materials zeigt sich, dass es inhaltliche Typen gibt, die ausschließlich oder vornehmlich entweder als gemalte, geritzte oder gemeißelte Inschriften geschaffen wurden. Da diese Beziehungen für alle hier behandelten Fragen von Bedeutung sind, sollen die verschiedenen Untergruppen des Materials im Folgenden knapp vorgestellt werden. Außer lateinischen Inschriften wurde in Pompeji und Herculaneum auch eine deutlich geringere Zahl an oskischen Inschriften gefunden, die bei Emil Vetter und Helmut Rix zusammengestellt sind. ${ }^{133}$ Von diesen sind die Nummern Ve 58-69 Graffiti, Ve 23-35 Dipinti und Ve 8-22 in Stein gemeißelte Inschriften.

\subsubsection{Stein- und Metallinschriften}

Für die gesamte Antike am breitesten belegt sind Inschriften, die in Stein gemeißelt wurden. Besonders auf diese Materialgruppe und die Praxis des öffentlichen Ausstellens steinerner Textmonumente nimmt Ramsay MacMullen mit dem Begriff „Epigraphic Habit“ Bezug. ${ }^{134}$ In Pompeji und Herculaneum sind insgesamt 448 Stein- und Metallinschriften bekannt. ${ }^{135}$ Diese lassen sich inhaltlich in neun Gruppen unterteilen: Grabinschriften, Ehreninschriften, Bau- und Stifterinschriften, Weihinschriften, Dekrete oder Urkunden, Listen von Kultpersonal oder Beamten etc. sowie Datumsangaben, Elogien und weitere Inschriften, die aufgrund des Wortlauts oder des Erhaltungszustandes keiner der genannten Kategorien zugeordnet werden können. Die Unterscheidung zwischen Weih- und Stifterinschriften ist nicht immer eindeutig. Bei den Grabinschriften ist hingegen eine weitere Differenzierung in Grabtituli und weitere zu den Bestattungen gehörende Inschriften möglich. Die meisten Steinund Metallinschriften waren im öffentlichen oder halböffentlichen Raum der Städte

133 Vetter 1953, 46-67 unter den Nummern Ve 8-72e. Hinzu kommen wie bei den lateinischen und griechischen Inschriften Ergänzungen, die durch fortdauernde Grabungen möglich werden, z. B. De Caro 1983. Siehe auch Rix 2002, 103-113 unter den Nummern Po 1-101 und die Ziegelstempel tPo 1tPo 43. Bei Rix sind 36 oskische Inschriften aufgeführt, die Vetter noch nicht kannte.

134 MacMullen 1982.

135374 in Pompeji und 74 in Herculaneum.

Ә Open Access. ( 2021 Fanny Opdenhoff, publiziert von De Gruyter. (c) BY-NC-ND Dieses Werk ist lizenziert unter der Creative Commons Attribution-NonCommercial-NoDerivatives 4.0 Lizenz.

https://doi.org/10.1515/9783110722758-002 
aufgestellt oder angebracht. Hinzu kommen solche, die innerhalb von Wohnhäusern angebracht waren und solche, die in sekundären Kontexten gefunden wurden, jedoch ehemals vermutlich im öffentlichen Raum ausgestellt waren.

Die größte Gruppe unter den Steininschriften stellen die Grabinschriften dar. In Herculaneum sind aufgrund der Ausgrabungsbedingungen lediglich vier Grabinschriften bekannt. ${ }^{136}$ In Pompeji dagegen, wo die Ausfallstraßen zu signifikanten Teilen freigelegt werden konnten, kommen 150 Exemplare hinzu. Die außen angebrachte Grabinschrift, der titulus, ist nicht an allen Gräbern erhalten: einmal in Herculaneum und 60 mal in Pompeji. Die überlieferten Exemplare sind unterschiedlich ausführlich, wobei kein direkter Zusammenhang zwischen dem Textumfang und der Pracht des Monumentes besteht. ${ }^{137}$ Als zwei extreme Beispiele seien die Grabinschrift vom Grab der Eumachia in der Nekropole an der Porta di Nocera (OS 11): Eumachia // sibi et suis und die Inschrift CIL X 1030 am Grabmal des Caius Munatius Faustus und der Naevoleia Tyche genannt: Naevoleia L(uci) lib(erta) Tyche sibi et / C(aio) Munatio Fausto Aug(ustali) et pagano / cui decuriones consensu populi / bisellium ob merita eius decreverunt / hoc monimentum Naevoleia Tyche libertis suis / libertabusq(ue) et C(ai) Munati Fausti viva fecit. Während die Eumachii sich auf die absolut notwendigen Angaben, nämlich welcher Familie das Grab gehörte und wer darin bestattet werden durfte, beschränkten, nutzte Naevoleia die Inschrift dazu, ausführlich auf den Status und die Leistungen ihres Mannes einzugehen. Hinzu kommen oft Angaben zur Größe des Grabbezirkes oder zu den Kosten für die Errichtung des Monuments.

Außer den tituli, die in ihrer Anbringung auf das Monument bezogen sind und meist als Platten gut sichtbar in die Fassade oder an anderer Stelle eingelassen wurden, tragen auch Stelen in Hermenform sowie andere kleinere Monumente Inschriften. Diese bezogen sich direkt auf die einzelne Bestattung und nennen den Namen und häufig das Alter der Verstorbenen. Solche Stelen finden sich auch ohne Beschriftung. Sie dienten in der Regel als Markierung der Stelle, an der die Aschenurne vergraben war und wo Libationen vorgenommen werden konnten. ${ }^{138}$ Wo die Fundstelle bekannt ist, liegt diese meist innerhalb der Grabbezirke, sodass sie nicht zu den Inschriften im öffentlichen Raum zu zählen sind.

Als zweite große Gruppe kommen Ehreninschriften vor. Mit 39 Exemplaren fallen mehr als die Hälfte der Inschriften aus Herculaneum in diese Kategorie. In Pompeji kommen weitere 37 hinzu. Die elementaren Bestandteile sind der Name des Geehrten sowie eine Formel, die die Rechtmäßigkeit der Aufstellung des Monuments, an dem

136 CIL X 1473-1475. 1477 sowie ohne Kontext: CIL X 1468. Die Ausgrabungen in Herculaneum konzentrieren sich auf den innerstädtischen Bereich und umfassen auch dort nur wenige insulae. Grabkontexte wurden bisher nur vereinzelt erforscht: Cochin/Bellicard 1755, 24-28. Taf. 6; Ruggiero 1885, XXXVII-XXXVIII; Maiuri 1958a, Taf. 2; Pagano 1989; Pappalardo 1997; Parslow 1995, 335 Anm. 16; 338 Anm.3.

137 Campbell 2015, 61-69. 110-133.

138 Kockel 1983, 16-18. 
sie angebracht waren, bestätigt. Der Begriff „Ehreninschrift“ wird hier im Sinne von „Inschrift an einem oder in direktem Zusammenhang mit einem Ehrenmonument“ verstanden. Wie Eck zurecht betont, kommen solche Inschriften in der Antike nicht isoliert vor, sondern sind Teile von Monumenten, die aus unterschiedlichen Komponenten bestehen. ${ }^{139}$ In Herculaneum lässt sich anhand dieser Inschriften feststellen, dass sich eine kleine Gruppe von Bürgern besonders stark für das Gemeinwohl engagierte und dafür zahlreiche Ehrungen erhielt. Darunter stechen Lucius Mammius Maximus und noch stärker Marcus Nonius Balbus hervor. ${ }^{140}$

Bau- und Stifterinschriften sind besonders in Pompeji mit 53 Exemplaren zahlreich vertreten. In Herculaneum sind 14 belegt. Besonders die größeren Bauprojekte wie das Eumachiagebäude oder der von Marcus Tullius gestiftete Fortuna AugustaTempel, die auf die Initiative einzelner Bürger zurückgingen (Abb.F2), aber auch Reparaturarbeiten oder Ausbauarbeiten an bereits bestehenden Gebäuden wurden in Inschriften memorialisiert. ${ }^{141}$ Besonders bekannt ist die Inschrift des Numerius Popidius Celsinus am Iseum (CIL X 846), die auf dessen Engagement nach dem Erdbeben im Jahr 62 n. Chr, hinweist, und die Inschriften im Amphitheater, die die Stifter der steinernen Sitzreihen nennen (CIL X 853-857.). Es werden jedoch nicht nur solche Inschriften zu den Bau- und Stifterinschriften gezählt, die davon zeugen, dass ein Einzelner oder eine Gruppe auf Eigeninitiative ein Bauwerk konzipiert und finanziert hatte. Auch Inschriften von Architekten, die an Bau- oder Renovierungsarbeiten beteiligt waren, sind in diesem Kontext zu berücksichtigen. ${ }^{142}$ Darüber hinaus können auch Ehreninschriften zugleich Stifterinschriften sein, wenn darin derjenige namentlich genannt wird, der für das Monument aufkam. ${ }^{143}$

Auch die Abgrenzung zu Weihinschriften ist nicht immer einwandfrei möglich oder sinnvoll. Nicht immer sind Formulierungen wie votum solvit, sacrum, ex visu oder ex iussu erhalten, sodass gegebenenfalls nur aus dem Kontext erschlossen werden kann, dass es sich um eine Weihung an eine Gottheit handelt. ${ }^{144}$ Da auch dies aufgrund der Dokumentationslage nicht immer möglich ist, muss die Trennung zwischen Stifter- und Weihinschriften etwas unscharf bleiben. In Pompeji können der Kategorie der Weihinschriften unter diesem Vorbehalt 43 Inschriften zugewiesen werden, in Herculaneum hingegen nur zwei.

139 Eck 1999, 67.

140 Mammius: CIL X 1449. 1452. Nonius Balbus: CIL X 1426-1434. Besonders herausragend sind da Ehrenmonument für Balbus und die ebenfalls als Ehreninschrift zu verstehende Inschrift an seinem Grabmonument auf dem Platz vor den Terme suburbane: AE 1990, 164; AE 1976, 144. Vgl. Camodeca 1982, 125-126.

141 Stifterinschriften am Eumachiagebäude: CIL X 810 und 811; Stifterinschrift am Fortuna AugustaTempel: CIL X 822.

142 In Pompeji sind dies CIL X 841 und 868.

143 So etwa die fullones für die Statue der Eumachia in CIL X 813.

$144 \mathrm{Zu}$ den Formeln in Weihinschriften siehe besonders: Raepsaet-Charlier 2001. 
Neben diesen zahlreich vertretenen Inschriftenkategorien kommen in Pompeji und Herculaneum 13 weitere Inschriften unterschiedlichen Inhalts vor. Darunter sind besonders die Stelen des Titus Suedius Clemens von Bedeutung (Abb. 52. 53. 54. F69), die zu den Dekreten und Urkunden zu rechnen sind. Für die Geschichte der Grabungen sind sie von großer Bedeutung, da erst mit dem Fund der Stele in der Nekropole vor der Porta Ercolano (CIL X 1018) die Stadt sicher mit Pompeji identifiziert werden konnte. Da die drei in situ gefundenen Exemplare in einer Entfernung von ca. $30 \mathrm{~m}$ von der Linie der Stadtmauer entfernt aufgestellt wurden, ist es zudem sehr wahrscheinlich, dass sie die Grenze des pomerium oder eine vergleichbare Pufferzone um die Stadt herum markierten. ${ }^{145}$ In Herculaneum kommen zwei Dekrete hinzu, ein inzwischen verschollenes senatus consultum sowie das Dekret am Altar des Marcus Nonius Balbus. ${ }^{146}$

Unter den Steininschriften kommen zudem Listen von Trägern kultischer oder politischer Ämter vor. ${ }^{147}$ Eine weitere Liste stellt das große album aus Herculaneum dar. Davon wurden bereits bei den bourbonischen Grabungen elf Fragmente gefunden und 1883 unter der Nummer 1403 im CIL X veröffentlicht. In den 70er Jahren des 20. Jh.s kamen weitere vier Fragmente hinzu. ${ }^{148}$ Der Text beinhaltet unter Rubriken zusammengefasste Namenslisten verschiedener Stände. Sehr wahrscheinlich handelt es sich dabei um Einwohner der Stadt. Die These, dass hier die augustales aufgelistet seien, wird mittlerweile jedoch allein wegen der hohen Anzahl an Namen zurückgewiesen. ${ }^{149}$

Hinzu kommen zwei Inschriften, deren Nachbildungen heute am Westeingang des Gebäudes der Eumachia angebracht sind. Diese können aufgrund des Inhaltes am ehesten als enkomia auf Aeneas und Romulus bezeichnet werden und sind in Pompeji und Herculaneum singulär. ${ }^{150}$ Mit nur drei Vertretern kommen in Pompeji zudem in Stein gemeißelte Datumsangaben vor: CIL IV 1622 K Q (Abb.F4), 2307 ex K QVI (Abb. F4) und CIL IV 3339 ex K QVI. CIL IV 2307 befindet sich an der senkrechten Seite des Bordsteins an der Kreuzung von Via Stabiana und Via di Nola, CIL IV 1622 im Straßenpflaster des Vicolo Storto und CIL IV 3339 im Bordstein der nicht benannten Straße, die die Fortsetzung der Via degli Augustali bildet. 72 weitere Stein- und Metallinschriften können keiner dieser Kategorien zugewiesen werden, da nur geringe Reste der Texte übrig sind und auch die Fundstellen keine eindeutigen Rückschlüsse erlauben.

145 Kockel 1983, 4. Die anderen drei cippi wurden ebenfalls alle außerhalb der Stadt und zwar im Bereich der Porta Marina, vor der Porta Vesuvio und vor der Porta Nocera gefunden. Die Stele vor der Porta Marina befand sich bei der Auffindung aber in den Terme Suburbane. Vgl. Sertà 1998, 229. 234 Anm. 3; Sertà 2002, 233.

146 CIL X 1401. 1453.

147 CIL X 892 und 924 in Pompeji.

148 AE 1978, 119a-d.

149 Vgl. Camodeca 2008 und de Ligt/Garnsey 2012.

150 CIL X 808. 809. 
Bei den oskischen Steininschriften handelt es sich um Bau- und Weihinschriften, die von den zuständigen Beamten gesetzt wurden, wobei die Ämter des mediss túvtiks, des kvaísstur und des aídilis belegt sind. ${ }^{151}$ Alle oskischen Inschriften in Pompeji und Herculaneum sind im oskischen Alphabet geschrieben, das nicht nur den Gebrauch anderer Buchstaben, sondern auch die Lese- und Schreibrichtung von rechts nach links vorsieht. ${ }^{152}$ Aus Herculaneum ist eine Steininschrift bekannt, die bei Vetter unter der Nummer 107, aufgenommen ist. Dabei handelt es sich um eine Weihinschrift auf einem Altar. 153

\subsubsection{Dipinti}

Mit dem Begriff „Dipinti“ werden Inschriften bezeichnet, die mit flüssiger Farbe geschrieben wurden. Insbesondere in Pompeji sind sie für den Besucher auch heute noch kaum zu übersehen, obwohl im Laufe der letzten 250 Jahre viele der Wandinschriften verlorengegangen sind (Abb. F5). Da das Spektrum in Pompeji und Herculaneum anders als bei den Steininschriften sehr unterschiedlich ist, werden die beiden Städte getrennt beschrieben.

Die mit ca. 2600 Exemplaren größte inhaltlich klar abgrenzbare Gruppe unter diesen stellen die programmata dar. Dabei handelt es sich um Wahlempfehlungen für mindestens einen und bis zu vier Bewerber um die je zwei Posten der aediles, der IIviri und alle fünf Jahre der IIviri quinquennales. ${ }^{154}$ Die programmata zeichnen sich durch einen hohen Grad an Formalisierung aus, den vor allem Chiavia und Mouritsen detailliert beschreiben. ${ }^{155}$ Der einzige wirklich zwingende Bestandteil war der Name des Kandidaten, im Normalfall wurde aber zumindest auch das Amt genannt. Hinzu kommen zusätzliche Aspekte des Amtes, allgemeine Verweise auf die Qualitäten des oder der Kandidaten (DRP für dignum rei publicae und $V B$ für virum bonum), eine explizite Aufforderung zur Wahl (OVF für oro vos faciatis oder der Name eines Unterstützers mit dem Zusatz rogat) und seltener die Angabe SCR für scripsit/scripserunt und der Name des oder der Schreiber. Für alle Bestandteile außer den Namen hatte man konventionelle Abkürzungen gefunden, die entweder getrennt oder sogar als nexus wie eine Art neues Graphem eingesetzt wurden. Dadurch und insbesondere durch die regelhafte

151 mediss túvtiks: Ve 8.13.14. 15; kvaísstur: Ve 11.12.16.17.18. 19; aídilis: 8. 9. 10. 20.

152 Vgl. Vetter 1953, 1; Zum oskischen Alphabet: Rix 2005, 325. An anderen Orten werden Texte in oskischer Sprache auch in lateinischer, griechischer und etruskischer Schrift geschrieben.

153 Vgl. Vetter 1953, 90; Rix 2002, 116 unter der Nr. Cm 10.

154 Sie sind zugleich auch die am intensivsten erforschte Textgattung innerhalb der pompejanischen Epigraphik. Bereits 1887 bildeten sie die Grundlage für Pierre Willems’ Untersuchung der Wahlen in Pompeji: Willems 1887. Bis heute dienen sie immer wieder als Ausgangspunkt für Überlegungen zur politischen Struktur und der Organisation des Wahlvorgangs.

155 Chiavia 2002, 47. 82-85; Mouritsen 1988, 31. 
Verwendung des Kandidatennamens im Akkusativ sind die programmata auf den ersten Blick als solche zu erkennen.

Als weitere große Gruppe stechen besonders die 83 edicta munerum hervor, mit denen Gladiatorenkämpfe und andere Spektakel in Pompeji und den umliegenden Städten angekündigt wurden. Sie nannten meist den Veranstalter und gegebenenfalls den Anlass, den Ort, das Datum und die Attraktionen, die geboten wurden: Gladiatoren, venationes, Sonnensegel und Ähnliches. Der Veranstalter, der Anlass oder auch die Zahl der Gladiatoren wurden in der Regel in der ersten Zeile und in sehr großen, bis zu $70 \mathrm{~cm}$ hohen Buchstaben genannt. Darunter folgten die Details in kleinerer Schrift. Sabbatini Tumolesi erstellte zum ersten Mal eine inhaltlich orientierte Typologie dieser Texte, die auch die Identifikation fragmentarisch erhaltener edicta erlaubt. ${ }^{156}$ Diese Texte sind ebenfalls stark formularisiert, weisen aber eine breitere Variation auf, da es weitaus mehr Faktoren gab, die sich je nach Veranstaltung, Veranstalter, Ort und Zeit unterschieden.

Auch Grüße kommen mit 42 Belegen unter den Dipinti vergleichsweise häufig vor. Gekennzeichnet sind sie durch Ausdrücke wie feliciter, vale oder salutem. Sie benennen den oder die Gegrüßten im Dativ. Nur selten wird auch ein Grüßender namentlich benannt. ${ }^{157}$ Bei den Gegrüßten handelt es sich zum einen um Angehörige der lokalen Oberschicht, zum anderen um Auswärtige. Besonders interessant ist die mehrfache Erwähnung des Suedius Clemens, der vor allem durch die Setzung der cippi anlässlich der Rückführung der loca publica an das Gemeinwesen bekannt ist. An ihn sind zumindest drei Grüße gerichtet. ${ }^{158}$ Es kommen jedoch auch Kollektive wie der ordo $^{159}$, die defensores coloniae ${ }^{160}$ oder die Pompeiani ${ }^{161}$ vor sowie die iudiciis Augusti bzw. Augustis und Augustorum als Adressaten vor. ${ }^{162}$ Die Namen sowie die anderen Bestandteile der Grüße wurden meist nicht abgekürzt, sondern ausgeschrieben.

Eine kleine Gruppe, die unter anderem deshalb interessant ist, weil sie Parallelen an anderen Stätten findet, stellen die Topos-Inschriften dar, die am Amphitheater gefunden wurden. Sieben derartige Dipinti wurden in Pompeji aufgenommen. ${ }^{163}$ Der Text von CIL IV 1196, der hier beispielhaft wiedergegeben wird, lautet permissu / aedilium Cn(aeus) / Aninius Fortu/natus occup(avit): „Mit Erlaubnis der Aedilen hat Cnaeus Aninius Fortunatus [diese Nische] besetzt“. Die Texte der anderen fünf Dipinti

156 Sabbatini Tumolesi 1980, 116-119.

157 CIL IV 1101. 7243. 7989b.

158 CIL IV 7203. 7579. 7780.

159 CIL IV 7687.

160 CIL IV 7342.

161 CIL IV 3765. 7343.

162 Letztere: CIL IV 528. 1074. 3525. 3726. 7625.

163 CIL IV 1196. 1196a. 1196b. 1197. 1197a. 1197b. 2996. Möglicherweise sind dazu auch CIL IV 1115. 1768 und 1769 zu zählen. Vgl. Gassner 1986, 15. Weder Spuren oder Reste im Befund noch Abbildungen dieser Dipinti existieren. Parallelen (allerdings Steininschriften) finden sich z. B. in Corduba (CIL II 2242) und Thubursicu Numidarum (AE 1915, 85). 


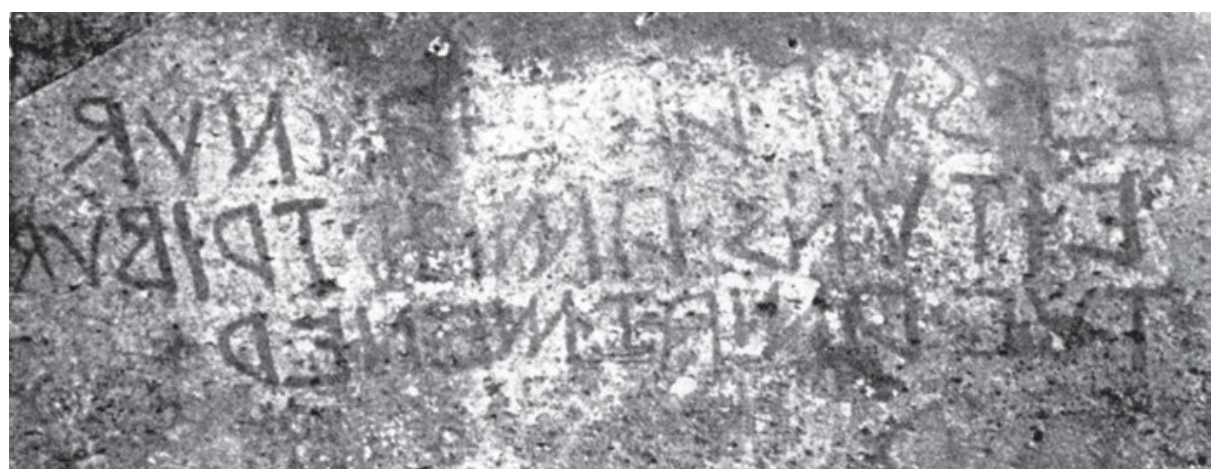

Abb. 2: eítuns-Inschrift Ve 27 zwischen den Eingängen VIII 5, 19 und 20. (C) Su concessione del Ministero per i Beni e le Attività Culturali e per il Turismo - Parco archeologico di Pompei. Jegliche weitere Reproduktion oder Duplikation ist untersagt.

sind nur fragmentarisch erhalten, lassen aber jeweils entsprechende Ergänzungen zu oder sind knapper formuliert.

Unter den Werbeinschriften (abgesehen von den programmata) aus Pompeji sind vor allem zwei Mietannoncen bekannt, die auf die Fassaden der Insula Arriana Polliana und der praedia Iuliae Felicis gemalt worden waren. ${ }^{164}$ Das inhaltliche Spektrum der Dipinti reicht hier weiterhin von Listen ${ }^{165}$ über Bekanntmachungen bis hin zu informellen Aufforderungen einzelner Einwohner. ${ }^{166}$

Die in oskischer Sprache verfassten gemalten Inschriften umfassen die sechs bekannten eituns-Inschriften (Ve 23-28) (Abb.2), ${ }^{167}$ bis $\mathrm{zu}$ zehn programmata (Ve 29-30h) und eine Reihe weiterer Inschriften (Ve 31-35), von denen mit Bezug auf den öffentlichen Raum Ve 33 relevant ist. Unter dieser Nummer werden fünf Inschriften zusammengefasst, die mit immer demselben Text, vaamunim‘ auf fünf Säulen am forum geschrieben waren. ${ }^{168}$ Eine enge inhaltliche Überschneidung mit den lateinischen gemalten Inschriften ergibt sich unter den oskischen Dipinti nur

164 CIL IV 138. 1136. Vgl. Pirson 1999, 15-21.

165 Dabei handelte es sich um weiß grundierte Tafeln, die mit Bekanntmachungen, Gesetzen oder Namenslisten verschiedener Räte oder Kollegien beschrieben und dann öffentlich ausgestellt wurden: Schmidt 1894, Sp.1332-1336; Sachers 1932, Sp.1881-1884; Liv. 9,46,5; Eck 1998, 211-215. Eck 1999, 5860. Dazu auch: Rea 1977, 153. Inhaltlich dieser Gruppe zuzuordnen sind jedoch CIL IV 60, eine Liste der magistri vici et compiti, die auf einen Tuff-Pfeiler im westlichen Abschnitt der Via dell'Abbondanza geschrieben war. Vgl. Eintrag im CIL IV 60. Eine genauere Lokalisierung ist anhand von Fiorellis dort abgedruckter Beschreibung nicht möglich. Siehe auch CIL IV 7807. Bei der letzteren handelt es sich wahrscheinlich ebenfalls um eine solche Liste, die jedoch auf den Putz an der SO-Ecke der insula IX 7 bei Eingang 1 gemalt worden war.

166 Dazu zählen besonders die Verkaufsangebote und cacator-Inschriften.

167 Diese gehören zu den meist diskutierten oskischen Inschriften überhaupt. Vgl. Antonini 2004; Sakai 1992; de Waele et al. 2001, 312-314.

168 Vgl. dazu CIL IV 674: victor, dessen V direkt unterhalb des oskischen V stand. 
bei den programmata. Während die eituns-Inschriften sehr wahrscheinlich aus der Zeit vor der Koloniegründung stammen und in engem Zusammenhang mit dem Bundesgenossenkrieg stehen, sind die oskischen programmata eher in den ersten Jahren nach der Eroberung zu kontextualisieren. Das Amt, das in den oskischen programmata genannt wird, ist das des IIIIner, dessen lateinische Entsprechung quattuorvir in Pompeji zwar gänzlich unbekannt, das aber auch in den oskischen Inschriften, die aus der Zeit vor $89 \mathrm{v}$. Chr. stammen, nicht belegt ist. ${ }^{169}$ Sie nehmen also ebenso wie die lateinischen programmata antiquissima, die das Amt des quaestor nennen, Bezug auf ein Amt, das für die Kaiserzeit in Pompeji nicht mehr zu fassen ist und möglicherweise nur in den frühen Jahren der Kolonie existierte. ${ }^{170}$

In Herculaneum sind die beiden größten pompejanischen Gruppen, die programmata und die edicta munerum, interessanterweise überhaupt nicht (sicher) belegt. ${ }^{171}$ Insgesamt ist die Anzahl an Dipinti in Herculaneum mit nur 19 gemalten Wandinschriften im CIL und ca. fünf Funden nach Abschluss des dritten Supplementes des CIL deutlich geringer als in Pompeji. ${ }^{172}$ Unter diesen insgesamt 24 Dipinti sind nur 13 sicher im öffentlichen Raum zu verorten, während der Rest nach den Beschreibungen des CIL in Innenräumen gefunden wurde. ${ }^{173}$ Das inhaltliche Spektrum ist schwierig $\mathrm{zu}$ fassen, da einige der Inschriften nur fragmentarisch erhalten sind. Der Dipinto Nola mit zwischen $L$ und A eingeschriebenem scr(iptor?) / Aprilis a / Capua (AE 1989, 182b) ist vermutlich ein edictum muneris. Bei den von Mario Pagano 1992 beschriebenen Fragmenten handelt es sich sicher um Namenslisten oder alba. Zwei Anschläge von lokalen Beamten am castellum aquae sind sowohl inhaltlich als auch technisch und gestalterisch interessant. In beiden geht es um eine Regelung des Verhaltens in der Umgebung des Wasserturms. CIL IV 10488 ist heute nicht mehr vorhanden, da es direkt über CIL IV 10489 gemalt war und von Della Corte gezielt entfernt wurde. Die herculanensischen Dipinti sind somit deutlich verschieden vom Gros der pompejanischen Zeugnisse. Dies kann sicherlich nicht nur auf die unterschiedlichen Konservierungsbedingungen zurückgeführt werden, da der Erhaltungszustand des Wandverputzes und der Bemalung von Wänden in Herculaneum nicht grundsätzlich schlechter ist als in Pompeji. Vielmehr sind die Gründe in unterschiedlichen gesellschaftlichen Gegebenheiten und in einem anderen Umgang mit Schriftlichkeit zu suchen. ${ }^{174}$

169 Mouritsen 1988, 89; Cooley 2002, 82-83.

170 Degrassi 1967.

171 Die Benennung eines 1967 entdeckten Dipinto am decumanus maximus von Herculaneum als programma kann nicht als sicher gelten. Vgl. dazu: Pagano 1987, 151-152. Dagegen: Solin 1973a.

172 CIL IV 1176. 3880. 10478-10494. Funde nach Abschluss des Supplementes: Pagano 1987; Pagano 1988b; Pagano 1992, 189-191: hier handelt es sich um fünf Fragmente von gemalten alba. Wie vielen separaten Listen diese zuzuordnen sind, ist unklar.

173 Im öffentlichen Raum befanden sich die Neufunde sowie CIL IV 10479. 10484. 10486-10489. 10491. 10493.10494.

174 Vgl. dazu: Solin 1973a, 101-102 und Wallace-Hadrill 2011a, 292. 


\subsubsection{Graffiti}

Bei den Steininschriften und bei den Dipinti konnte das inhaltliche Spektrum recht einfach beschrieben werden, da die Anzahl der Textsorten überschaubar ist. Bei Graffiti sieht man sich dagegen einer wesentlich größeren inhaltlichen Bandbreite gegenüber. ${ }^{175}$ Die inhaltliche Vielfalt ist geradezu bezeichnend für diese Gattung. Anders als Steininschriften und die meisten Dipinti standen sie nicht in einem direkten Zusammenhang von Auftrag und Institution, sondern wurden von vielen verschiedenen Akteuren ohne Bezugnahme auf eine Autorisierung in ganz unterschiedlichen räumlichen Kontexten angebracht. ${ }^{176}$ Dass das Graffitischreiben grundsätzlich als deviant betrachtet wurde, ist allerdings eine anachronistische Annahme. ${ }^{177}$ Anhand der Graffiti lassen sich äußerst vielfältige Formen des Umgangs mit Schrift und der Fähigkeit zu schreiben in verschiedenen sozialen Feldern feststellen.

Die heutige öffentliche Wahrnehmung der pompejanischen und herculanensischen Graffiti konzentriert sich vor allem auf im weitesten Sinne erotische Texte und Zeichnungen sowie auf geistreiche Epigramme und Zitate bekannter Textstellen aus der lateinischen Literatur. Dieses Interesse ist zwar verständlich, findet jedoch seinen Ursprung viel eher in heutigen Erwartungen als im Anteil der entsprechenden Texte an der Gesamtzahl der Graffiti. ${ }^{178}$ Das inhaltliche Spektrum der Graffiti ist vielmehr sehr breit und umfasst Grüße, einzelne Namen, Notizen, Epigramme, Diffamierungen, Handwerkersignaturen, Alphabete, Zahlenreihen, Datumsangaben, einzelne Buchstaben, Verweise auf bekannte Personen wie Gladiatoren sowie Nachrichten im engeren Sinne. Eine Auflistung dieser inhaltlichen Gruppen hat Langner am Beispiel einer Reihe von insulae und Häuser, deren epigraphische Zeugnisse großteils von Della Corte dokumentiert wurden, unternommen. ${ }^{179}$ Bei den meisten Graffiti handelt es sich um sehr kurze Texte, die inhaltlich deutlich weniger beziehungsreich sind als die meisten Steininschriften und Dipinti. Langner stellt fest, dass mehr als die Hälfte der Graffiti aus nur einem Wort besteht und dass weniger als $10 \%$ eine Verbalform enthalten. ${ }^{180}$ Dabei zeigen sich geringfügige Überschneidungen. Unter den Graffiti

175 Langner 2001, 21-26. Auf regionale Unterschiede weisen Jennifer A. Baird und Claire Taylor hin: Baird/Taylor 2011, 2.

176 Dieser im Vergleich mit den Steininschriften und Dipinti kaum regulierte Charakter der Graffiti wurde selbstverständlich schon vielfach hervorgehoben. Dazu unter anderen: Solin 1979, 278 sowie besonders zu diesem Aspekt: Voegtle 2012, 106.

177 Für eine differenziertere Betrachtung plädieren auch Baird/Taylor 2011, 3-4, Benefiel 2011, 20 und Langner 2001, 20.

178 Dies haben sowohl Martin Langner als auch Andrew Wallace-Hadrill bereits betont: Langner 2001, 22; Wallace-Hadrill 2011b, 410. Vgl. Solin 1979, 284-285.

179 Langner 2001, 21-24. Vgl. dazu auch die quantitative Auswertung bei Lohmann 2018, 136-142.

180 Langner 2001, 22-23. 
sind Wahlempfehlungen zu finden und unter den Dipinti wiederum tauchen vereinzelt bloße Namensnennungen auf. ${ }^{181}$

Außer textuellen Graffiti stellen auch verschiedenste bildliche Darstellungen einen Teilbereich dieses Materials dar. Langner unterscheidet in seiner Auflistung der inhaltlichen Gruppen textueller Graffiti zwischen Außen- und Innenräumen. Dabei wird deutlich, dass grundsätzlich die gleichen Inhalte in einem ähnlichen Verhältnis vorkommen. ${ }^{182}$ Ein deutlicher Unterschied ist jedoch zum einen bei Zahlen und Haushaltsnotizen zu erkennen, die in Innenräumen viel stärker vertreten sind. Zum anderen wurden Grüße, tituli memoriales ${ }^{183}$, Namensnennungen und Diffamierungen öfter im öffentlichen Außenraum als innerhalb von Häusern angebracht. ${ }^{184}$

Die oskischen Graffiti in Pompeji beinhalten zum Teil Namen (Ve 59c, möglicherweise Ve 61) und Alphabete (Ve 69a-1). Einige sind allerdings so disparat, dass keine inhaltliche Deutung möglich erscheint. Hinzu kommen sechs oskische Graffiti aus Herculaneum, von denen sich einer auf einem Ziegel fand ${ }^{185}$ und vier weitere im Vestibül der Casa Sannitica (V 1). ${ }^{186}$ Die bei Rix unter Cm 38 und Cm 39 aufgeführten Inschriften, die dem Eintretenden unmittelbar nach dem Überschreiten der Schwelle auf Augenhöhe begegneten, sind auch heute noch gut zu erkennen und sind als Grüße aufzufassen. ${ }^{187}$

\subsubsection{Zwischenergebnis}

Der Überblick über das inhaltliche Spektrum der Inschriften in Pompeji und Herculaneum erlaubt eine Kategorisierung der Inschriften sowie erste Aussagen über die beteiligten Akteure. Sowohl Steininschriften als auch Dipinti wurden für offizielle,

181 Dazu zählt CIL IV 10229b: Diocle(s?), welches mit der gleichen roten Farbe geschrieben ist, wie sehr viele der Dipinti.

182 Langner 2001, 22 (Außenräume), 23 (Innenräume), direkte Gegenüberstellung S. 24 Abb. 2. Auch bei Lohmanns Überblick über das inhaltliche Spektrum der Graffiti wird deutlich, dass kein grundlegender Unterschied zwischen Außen- und Innenräumen festzustellen ist: Lohmann 2018, 136-139.

183 Nach der Art aliquis hic fecit aliquid.

184 Dies sowie insbesondere auch die Verteilung der Bildthemen bei Graffitizeichnungen hebt auch Simone Voegtle hervor: Voegtle 2012, 109-110.

185 Rix 2002, 120 Nr. Cm 42.

186 Rix 2002, 119-120 Nrr. Cm 38, Cm 39, Cm 43 und Cm 44.

187 Zunächst war es nicht möglich, eine sichere Lesart zu etablieren. Vgl. Della Corte 1958b, 266; Antonini 1981, 336. Timo Sironen schlug überzeugend für beide Graffiti dieselbe Lesart vor: SALAVS L UVI[---], wobei salavs etwa mit salve zu übertragen wäre und L Úvi die Abkürzung eines Eigennamens sein muss: Sironen 1990, 459-461. Allerdings konnte Rosalba Antonini bei einer erneuten Autopsie 2006 feststellen, dass der Text um weitere fünf Zeilen zu ergänzen ist, die aufgrund der antiken und modernen Oberflächenbehandlung kaum mehr auszumachen sind. Antonini 2007, 86; 87 Taf. IV-V; 93 Abb. 9. Dabei handelte es sich anscheinend um eine mehrfache Wiederholung - mit gelegentlichen Verschreibungen - des von Sironen gelesenen Textes (Antonini 2007, 94). Antonini konnte zudem die Abhängigkeitsverhältnisse der Repliken genauer differenzieren. Vgl. Antonini 2007, 100-101. 
durch städtische Organe legitimierte Bekanntgaben verwendet. Der offizielle Charakter findet entweder dadurch Ausdruck, dass ein städtisches Organ oder ein Gremium der Urheber der Inschrift war, oder durch Formulierungen, die auf die Legitimation direkt Bezug nehmen. In beiden Gattungen finden sich auch Inschriften semioffiziellen Inhaltes, die rechtliche Relevanz hatten oder auf offizielle Strukturen und Institutionen Bezug nehmen sowie Relevanz für größere Teile der Bevölkerung beanspruchen, aber auf die Initiative Einzelner zurückzuführen sind. $\mathrm{Zu}$ den letzteren zählen Grabinschriften, Stifterinschriften, programmata sowie edicta munerum. Bei den Graffiti kommen solche offiziell verankerten oder autorisierten Inhalte dagegen nicht vor. Weder wird auf eine Institution als Urheberin von Inschrift oder Textträger verwiesen, noch stehen die Texte in direktem Zusammenhang mit verwaltungstechnischen Belangen oder dem Gemeinwesen als Bezugspunkt. Die Inhalte rühren dagegen in der Regel von persönlichen Interessen, Erfahrungen und Beziehungen her. Häufig treten hier auch der Urheber oder ein intendierter Adressat als benennbare oder anonyme Individuen in Erscheinung. Schnittmengen lassen sich auch bei Graffiti und Dipinti feststellen. In den Graffiti werden Personen erwähnt oder sogar Formeln imitiert, die aus Steininschriften und Dipinti bekannt sind. Und bei den Dipinti sind mit den Grüßen wiederum Texte belegt, die vorrangig unter den Graffiti zu finden sind. Hinzu kommen Warnhinweise gegenüber Umweltverschmutzern und Werbeinschriften, die sehr stark an Einzelinteressen und die persönliche Initiative der Urheber geknüpft sind. Bei den Ehren- und Stifterinschriften, die meist innerhalb der Stadtmauern verortet sind, gibt es Überschneidungen und Differenzen in der räumlichen Verteilung. Ehreninschriften kommen vor allem an freistehenden Monumenten auf Platzanlagen und im Theater vor, während die Bau-, Stifter- und Weihinschriften direkt an den jeweiligen Gebäuden, Gebäudeteilen oder Weihgaben an den fora, den Theatern und dem Amphitheater angebracht sind oder waren.

Betrachtet man die drei größten Gruppen unter den Stein- und Metallinschriften mit Blick auf ihre räumliche Verteilung, kristallisieren sich ihre primären Funktionen deutlich heraus: In den Nekropolen dienten Inschriften der Kennzeichnung des Grabes und zusammen mit Bildern und der Gestalt des Monumentes der Selbstdarstellung der Grabinhaber. ${ }^{188}$ Ehreninschriften legitimierten die Aufstellung des Monumentes und setzten den Geehrten in Szene. ${ }^{189}$ Die Bau-, Stifter- und Weihinschriften an Gebäuden und Monumenten legten Zeugnis vom Urheber ab, der sich so als Euerget inszenieren bzw. in monumentaler Form festhalten konnte, wie er seinen Amtspflichten nachgekommen war. ${ }^{190}$ Auch die Dipinti und die Graffiti lassen sich inhaltlich mit bestimmten Absichten und Vorstellungen der Urheber und der Leser verbinden. Als

188 Vgl. Feraudi-Gruénais 2003, 55-56.

189 Vgl. besonders Bergemann 1990, 15-16; Alföldy 2001, 12.

190 Kritisch zur Verallgemeinerung des Begriffes ,Euergetismus“ im Kontext von Stifterinschriften: Pobjoy 2000, 77-84. Zur Trennung von Wohltätigkeit und Amtspflicht auch: Alföldy 1997, 293-294; Eck 1997, 324-325; Panciera 1997. 
Textsorten können sie mit verschiedenen kommunikativen Funktionen wie Appellen oder Informationen usw. in Verbindung gebracht werden. $\mathrm{Zu}$ diesen Funktionen, die vor allem von einer inhaltlichen lesenden Rezeption abhängen, kommen allerdings weitere Aspekte hinzu, die durch eine Untersuchung von Materialität und Gestaltung sowie der räumlichen Verteilung an Schärfe gewinnen.

\subsection{Materialität und Gestaltung der Schriftzeugnisse in Pompeji und Herculaneum}

In diesem Kapitel werden die Eigenschaften der pompejanischen und herculanensischen Inschriften bezüglich der Textträger, der verwendeten Materialien und Werkzeuge sowie der Schriftarten und der Gestaltung beschrieben. Folgende Fragen stehen dabei im Mittelpunkt: Welche Konventionen bestanden bei der Gestaltung von Texten und in welchem Verhältnis stehen diese zu den jeweiligen Inhalten? Welche Techniken und welche Werkzeuge wurden genutzt, um die Inschriften zu schaffen? Lassen sich daraus Informationen über den Kreis der Schreiber und gegebenenfalls Zeichner gewinnen? Wie verliefen die Planung und Anbringung einer Inschrift, welche Arbeitsschritte sind an den Befunden nachzuvollziehen? Zeigen sich anhand der materialen Beschaffenheit Formen der Rezeption oder Modi der Wahrnehmung von Geschriebenem im öffentlichen Raum?

\subsubsection{Stein- und Metallinschriften}

\subsubsection{Textträger}

Textträger von Stein- und Metallinschriften können je nach Gattung ganze Gebäude, Vorrichtungen oder Teile größerer Strukturen, freistehende Monumente oder aber Tafeln sein, die theoretisch mobil waren. Die Einbindung der Inschrift in die jeweilige Struktur hängt von der Art der Anbringung ab.

Die meisten Grabinschriften sind auf rechteckige Steinplatten geschrieben und mittig in die Front oder Fassade des Grabmonumentes eingelassen. Sie werden, soweit dies im Befund zu erkennen ist, an ihren Rändern durch den Putz, mit dem das gesamte Monument verkleidet war, überdeckt (Abb. F10). Bei Monumenten, deren Fassade mit einem Giebel gestaltet war, wurden die Inschriften im tympanon platziert. Doch je nach Bauform und Material waren auch andere Anbringungsmöglichkeiten gegeben. So konnte bei Grabbauten, die als Altar gestaltet waren, eine Seite des Altars als Schriftträger genutzt werden. ${ }^{191}$ Bei scholae bietet sich die Möglichkeit, ein

191 Z. B.: Nekr. Porta Ercolano: Süd 20 CIL X 1026 (Kockel 1983, 91); Süd 22 CIL X 1030 (Kockel 1983, 100). Nekr. Porta Vesuvio: Grab des Vestorius Priscus (Spano 1910, 402). 
weiteres Element wie einen Altar oder einen Sockel in den Scheitelpunkt des Kreisausschnittes einzulassen und die Inschrift daran anzubringen, oder aber den Text direkt in die Rückenlehne einzuschreiben wie an der schola der Mammia ${ }^{192}$ (P. Ercolano, Süd 4, Abb.F6). ${ }^{193}$ Auch freistehende cippi kommen bei Gräbern vor. Diese haben jedoch vor allem die Funktion, den jeweiligen Bezirk zu markieren. ${ }^{194}$

Bei Inschriften, die auf Tafeln aus Marmor oder aus einem anderen hellen Stein gefertigt sind und in die Fassade der Grabmonumente eingelassen wurden, zeigt sich, dass diese Fassaden meist optisch einen dem Stein ähnlichen Eindruck machten, da sie mit einem sehr feinen, hellen Putz versehen waren. Nicht selten wurde dabei der Putz oder Stuck durch plastische Gestaltung als Inkrustation gearbeitet, sodass die Wirkung der einer hochwertigen Verkleidung mit weißem Marmor ähnelte. Diese Gestaltungsmuster können auch heute noch gut in der Nekropole an der Porta di Nocera beobachtet werden, wo an der Nordseite der Straße nach Nocera eine ganze Reihe von Gräbern umfangreiche Spuren der antiken Verkleidung aufweist (Abb. 50). ${ }^{195}$ Daneben existieren in derselben Nekropole auch Grabmale wie OS 17, das Grab der Tillii, welches mit Tuffplatten verkleidet ist (Abb. F7). Hier wurde man am oberen Rand des Podiums eine Marmorplatte eingelassen, die sich farblich deutlich vom Rest des Grabes abhebt. ${ }^{196}$ Bei den hell verputzten Gräbern kann nie ausgeschlossen werden, dass sie teilweise oder ganz bemalt waren und für die Inschriften ist generell anzunehmen, dass die Buchstaben rot oder schwarz ausgemalt waren. Dennoch wird deutlich, dass man versuchte, durch die gewählten Materialien am gesamten Monument eine einheitliche Grundlage für weitere Gestaltungsmöglichkeiten zu bieten. ${ }^{197}$

192 Zur Schreibweise des Namens mit zwei <m> vgl. Kockel 1983, 58-59.

193 Z. B.: Nekr. Porta Ercolano: Süd 2 CIL X 998 (Kockel 1983, 51); Süd 4 CIL X 998 (Kockel 1983, 57); Nekr. Porta Nola: Grab der Aesquillia Polla (Spano 1910, 390); Nekr. Porta Stabia: Grab des Marcus Alleius Minius (Sogliano 1890, 330).

194 Nekr. Porta Ercolano: Süd 3 CIL X 997 (Kockel 1983, 53); Nekr. Porta Nocera: OS 15 (D’Ambrosio/ De Caro 1983, ohne Seitenzahlen, unter der Nummer der Grabes); Nekr. Porta Stabia: Grab des Marcus Tullius (Sogliano 1890, 329). Zur Deutung dieser Stelen: Spano 1910, 329-330. Es scheint sich dabei nicht um standardisierte Formulierungen zu handeln, da sich die Texte der drei Inschriften schon bei der Angabe des Grabinhabers unterscheiden. Bei Grab OS 15 in Nekr. Porta Nocera wird der Inhaber im Nominativ genannt, vor der Porta Ercolano im Genitiv und vor der Porta Stabia im Dativ. Vgl. dagegen die Standplatzzuweisungen am Amphitheater: Kap. 2.2.2.3.3, S.79.

195 EN 4, EN 6, EN 10, EN 12, EN 14. Für zahlreiche weitere Grabbauten kann anhand kleinerer Putzreste ein ähnliches Aussehen rekonstruiert werden, wie zum Beispiel für die Gräber OS 11 (Grab der Eumachia), OS 13 (Grab des Marcus Octavius) und OS 23 (Grab des Publius Vesonius Phileros. Vgl. dazu D’Ambrosio/De Caro 1983, unter den Einträgen zu den jeweiligen Gräbern.

196 Die Verkleidung mit Tuffplatten, die Form der Inschrift und Details der Formulierungen sprechen jedoch dafür, dass das Grab noch in das Ende des 1. Jh.s v. Chr. zu datieren ist, womit es zu den ältesten Grabmonumenten in dieser Nekropole zählt. Vgl. D’Ambrosio/De Caro 1983, ohne Seitenzahl unter Nr. OS 15.

197 Somit boten die Grabfassaden auch ideale Ausgangsbedingungen, um sie mit gemalten oder geritzten Inschriften zu beschreiben. Vgl. Cormack 2007, 594. 
Ehreninschriften waren in der Antike immer Teile von Monumenten. ${ }^{198}$ Die Anbringungsart und das Verhältnis zum Textträger waren daher am Aufbau des gesamten Monumentes ausgerichtet. In den meisten Fällen handelt es sich um einen Sockel mit einer Statue, deren Ausrichtung teilweise anhand der Standspuren rekonstruiert werden kann. Die Inschriften sind in diesen Fällen immer an der Seite angebracht, auf die auch die Statue frontal ausgerichtet war. Nur in Einzelfällen wurde das Monument so in ein bestehendes Bauwerk integriert, dass kein extra gearbeiteter Sockel verwendet wurde: Bekannt ist in Pompeji das Denkmal für die Holconii im großen Theater. Die Inschrift wurde in diesem Fall direkt in die Sitzstufen eingelegt und das eigentliche Monument darin verankert. ${ }^{199}$ Generell bedeutet dies, dass Ehreninschriften nicht als gesonderte Plaketten oder Tafeln, sondern unmittelbar in die Oberfläche des Monuments eingeschrieben wurden.

Im Unterschied zu den Grab- sowie den Ehreninschriften wurden Bau- und Stifterinschriften auf zwei unterschiedliche Arten angebracht. In den meisten Fällen begegnen sie eingemeißelt in Bauteile der Gebäude, auf deren Errichtung sie Bezug nehmen oder direkt an Gegenständen, deren Stiftung und Bereitstellung sie thematisieren. Andererseits kommen Tafeln vor, die separat gefertigt und in der Umgebung der errichteten Struktur aufgestellt wurden. Diesen Weg wählte man jedoch vor allem anlässlich von Straßenarbeiten und bei einzelnen anderen Großbauten, die keine klar erkennbare Front, Eingangssituation oder anderweitig prononciert hervorgehobene Anbringungsstelle boten. Typisch sind die oskische und die lateinische Stele im Umfeld der Porta Stabiana, die Straßenbauarbeiten innerhalb und außerhalb der Stadt memorieren (CIL X 1064 und Ve 8). Bei Reparaturmaßnahmen an bestehenden Gebäuden wurden Tafeln in die vorhandene Bausubstanz eingelassen. Diese Praxis findet sich am Iseum in der Inschrift des Numerius Popidius Celsinus (CIL X 846) oder in den beiden Inschriften CIL X 858 und 859 der Cuspii Pansae zu beiden Seiten des nördlichen Korridors im Amphitheater.

\subsubsection{Verwendete Materialien und Werkzeuge}

Im Folgenden soll geklärt werden, welche Materialien für die verschiedenen Steininschriften verwendet wurden und wodurch die Wahl der Steine und weiterer Stoffe bedingt gewesen sein konnte. Eine detaillierte petrographische Analyse der verschiedenen Inschriftenträger steht noch aus und konnte im Rahmen dieser Arbeit nicht durchgeführt werden. ${ }^{200}$ Daher dienen die Angaben aus dem CIL in Kombination mit weiteren Publikationen und der Autopsie als Grundlage der Beschreibung der

198 Vgl. Kap. 2.2.1, S. 35.

199 Bei dem Monument muss es sich, wie die Dübellöcher zeigen, um eine sella curulis gehandelt haben. Vgl. Fuchs 1987, 45 (B II 3) und Schäfer 1989, 133.

200 Für manche anderen Anwendungsbereiche liegen bereits Studien vor, wie zum Beispiel für die Marmorverkleidungen von Theken in einigen Läden: Fant et al. 2013, besonders 188. 
Materialien. ${ }^{201}$ Besonders häufig kommen demnach heller Kalkstein und Marmor als Material für Grab-, Ehren- und Stifterinschriften vor. Farblich auffällige Steinsorten wurden insgesamt vergleichsweise selten gewählt. Eine Ausnahme bildet die Verkleidung des Sockels des Ehrenmonuments für Quintus Sallustius an der O-Seite des forums mit einem bunten, vorwiegend roten Marmor (Abb. F8). ${ }^{202}$

Als Material für die Inschriftenplatten an Gräbern wurde meist heller, kompakter Stein und besonders häufig - bei 40 der 58 Inschriften - Marmor verwendet. Außerdem kamen Travertin, andere Kalksteinsorten und Tuff zum Einsatz, letzterer jedoch nicht in Form eingelassener Platten, sondern nur dann, wenn die Inschrift direkt auf die bauliche Substanz bzw. die Verschalung des Grabbaus geschrieben wurde, wie bei Grab OS 31 in der Nekropole an der Porta di Nocera (Abb. F9) oder an den scholae der Mammia (Abb.F6) und des Marcus Alleius Minius. Für die cippi wurde Lava verwendet. Insbesondere in den Nekropolen fällt auf, dass die Wahl des Steins für die Grabinschrift und des Materials für die Verkleidung des Monumentes korreliert waren. Hier finden sich oft helle Steine in weiß verputzen Flächen, wie zum Beispiel bei Grab EN 4 in der Nekropole an der Porta Nocera (Abb. F10).

Ehreninschriften wurden prinzipiell auch in Bronzetafeln geschrieben. In Herculaneum sind die drei Inschriften, die an den Ehrenmonumenten für Kaiser Claudius (CIL X 1416), für Lucius Mammius Maximus (CIL X 1452) und für Tetteiana (CIL X 1458) angebracht waren, aus diesem Material gefertigt. Ansonsten wurden alle Ehreninschriften in Pompeji und Herculaneum - soweit Informationen zu den verwendeten Materialien vorliegen - in Stein gemeißelt. Für die Inschriften wurden meist weiße oder hellgraue Sorten verwendet, wobei zu bedenken ist, dass Buntmarmor auch nach der Verschüttung ein besonders begehrtes Fundgut darstellte, sodass dieser heute vermutlich ähnlich wie die Bronzeeinlagen im Forumspflaster unterproportional erhalten ist. Ein Beispiel für die Verwendung von buntem Marmor bietet die Inschrift im Sockel für eine Reiterstatue des Quintus Sallustius (CIL X 792) am forum von Pompeji (Abb. F11). Hier hatte man für die Verkleidung des gesamten Sockels Pavonazzetto in den Farben dunkelgrau, hellgrau, rot, rosa und weiß gewählt. Die darin eingemeißelte Inschrift wurde rot ausgemalt, wovon noch substantielle Reste zu erkennen sind. An

201 In dieser Hinsicht informativ sind die Kataloge des Museo Archeologico Nazionale di Napoli, die Publikationen der Nekropolen vor der Porta Ercolano und der Porta Nocera in Pompeji sowie der kleine Band von Hüttemann: Fiorelli 1867; Fiorelli 1868; D’Ambrosio/De Caro 1983; Kockel 1983; Kockel 2005; Hüttemann 2010. Mommsen geht im CIL X nur ausnahmsweise auf die Beschreibstoffe ein. Nur bei CILX 997 findet sich der Hinweis, die Inschrift sei litteris vetustis in lapide molari (=Lava) geschrieben. Entsprechende Informationen bieten weiterhin Grabungsberichte und neuere Untersuchungen: Sogliano 1890, Spano 1910, De Caro 1979, D’Ambrosio/De Caro 1987, Emmerson 2010.

202 Die Inschrift auf dem Sockel lautet: Q(uinto) Sallustio P(ubli) F(ilio) / IIvir(o) i(ure) d(icundo) quinq(ennali) / patrono d(ecreto) d(ecurionum) (CIL X 792). Allerdings ist es fraglich, wie außergewöhnlich und auffällig dieser Sockel im Umfeld der deutlich größer dimensionierten Sockel an der S-Seite des forums wirken konnte, die bereits bei der Ausgrabung ihrer Verkleidungen beraubt waren. Vgl. Kockel 2005, 55. 
den Stellen, an denen sich Farbreste erhalten haben, sind die Buchstaben etwas besser zu erkennen, als es allein durch den Schattenwurf des Keilschnittes möglich wäre. Dennoch scheint die optisch heterogene Wirkung des Steines und insbesondere die teilweise rote Färbung für die Lesbarkeit des Textes eher hinderlich. Dies wurde jedoch in Kauf genommen, um das Monument durch die Wahl eines besonders auffälligen und auch durch seinen Import besonderen Stein aufzuwerten. ${ }^{203}$ Die verschiedenen hellen Marmorsorten, aber auch der feine, harte Kalkstein, der für die drei Sockel der Cuspii Pansae (Vater und Sohn) und des Marcus Lucretius Decidianus Rufus verwendet wurde, boten dagegen optimale Bedingungen, um den Inschriftentext sicht- und lesbar zu machen (Abb. F12).

Bei den meisten Bau-, Stifter- und Weihinschriften, die als Tafeln an einem größeren Bauwerk angebracht waren, ist $\mathrm{zu}$ beobachten, dass das Baumaterial des gesamten Monumentes auch für die Tafel verwendet wurde, oder dass - wie bei den Grabmonumenten - durch die Wahl der Materialien versucht wurde, einen optisch homogenen Eindruck zu erzielen. Die älteren lateinischen Inschriften bzw. die lateinischen Inschriften an Monumenten, die aus der Zeit unmittelbar nach der Koloniegründung stammen, sind in Travertin geschrieben. Dazu zählen der Eichtisch am forum (CIL X 793), die Inschrift der Beamten Caius Vulius und Publius Aninius bezüglich der Arbeiten an den Stabianer Thermen (CIL X 829) sowie die Bauinschriften an odeion und Amphitheater, die beide von Caius Quinctius Valgus und Marcus Porcius in den 70er Jahren des 1. Jh.s v. Chr. gebaut worden sein müssen (CIL X 844 und 852, je zwei gleichlautende Inschriften). Auch die Inschriften derer, die nach und nach die cunei des Amphitheaters zu steinernen Sitzstufen ausbauen ließen, schrieb man in die bereits bestehende Travertinbalustrade ein. Die meisten Bauwerke, die später und besonders ab augusteischer Zeit entstanden, sind dagegen mit Marmorschmuck ausgestattet und oft mit einem hellen Putz verkleidet. Die zugehörigen Inschriften wurden meist ebenfalls in Marmor gefasst. Dies gilt für die Bauinschriften des Eumachia-Gebäudes (CIL X 810. 811) ebenso wie für die des Fortuna Augusta-Tempels (CIL X 820), aber auch für die Tafel am Iseum, die die Renovierungsarbeiten nach dem Erdbeben bezeugt (CIL X 846). Ebenso verhält es sich bei den Stifterinschriften in Herculaneum und den beiden Tafeln, die die Hilfe von Seiten des Kaisers Vespasian nach einem Erdbeben im Jahr 76 n. Chr. bezeugen (CIL X 1406 und AE 1979, 170). Hinsichtlich der Materialität der Inschriften kann kein grundsätzlicher Unterschied zwischen kaiserlicher und nichtkaiserlicher Bautätigkeitfestgestellt werden. ${ }^{204}$ Wie Marietta Horster betont, orientierte sich die Wahl des Materials, aber auch die Größe

203 Dass dies vermutlich gar nicht so selten vorkam und wie beliebt diese optischen Effekte waren, zeigt die Untersuchung von Clayton Fant, Ben Russell und Simon Barker, insbesondere: Fant/Russell/ Barker 2013, 198, wonach sich unter den Buntmarmor-Fragmenten, die zur Verschönerung von Ladentheken verwendet wurden, auch zahlreiche Inschriftenreste fanden.

204 Vgl. z. B. die Inschrift der Vibidia und des Furius vom Venustempel in Herculaneum (AE 2008, 357). 
und die Gestaltung der Inschriften weniger am Status des Stifters, als vielmehr am Baumaterial des gesamten Gebäudes und an den Maßstäben, die in dessen Anlage gesetzt waren. ${ }^{205}$

Alle drei Markierungen bzw. Datumsangaben im Straßenpflaster bzw. Bordstein sind in Lavablöcke eingegraben. Zangemeister nennt bei CIL IV 3339 ein scalprum als Werkzeug. ${ }^{206} \mathrm{Ob}$ sie tatsächlich eingeritzt oder eingemeißelt wurden, ist nicht zu entscheiden. Da es sich um recht harte Steine handelt, ist eine Bearbeitung mit Hammer und Meißel jedoch wahrscheinlicher. Die Buchstabenformen sind schlicht und ohne jeden Schmuck.

Die oskischen Inschriften sind mehrheitlich in Travertin und Marmor gearbeitet. ${ }^{207}$ Es handelt sich um Tafeln, frei aufgestellte Monumente oder Platten, die meist keine Aussage über die Einbindung in den Kontext durch das Material erlauben. Eine Ausnahme bildet die Bauinschrift Ve 15 auf dem Architravblock eines kleinen Rundbaus vom Foro Triangolare. Bei diesem hing die Wahl des zu beschreibenden Materials natürlich direkt vom Baumaterial ab. Ähnlich verhält es sich bei dem kleinen Altar aus der Casa del Fauno (Ve 21) sowie der Inschrift auf der Altartischplatte aus Herculaneum (Ve 107) ${ }^{208}$ Hier wurde das Material unabhängig von der Inschrift gewählt und richtete sich nach der Funktion des Gegenstandes. Die Inschrift Ve 18, in der cella des Apollontempels ist aus einem ungewöhnlichen Material und von ungewöhnlicher Machart. Der Boden ist in einer Kombination aus opus sectile und Mosaik dekoriert. Zwischen dem opus sectile-Feld in der Mitte und dem Mosaik, welches dieses umgab, verläuft ein schmaler Streifen aus Schiefer, auf den die Inschrift geschrieben ist (Abb.3). Die einzelnen Linien sind mit kleinen, Metallpünktchen gebildet. ${ }^{209}$ Bei den übrigen Inschriften zeigt sich, dass der verwendete, sehr kompakte Travertin für eine Beschriftung sehr gut geeignet war und wie Marmor eine präzise Ausarbeitung der Buchstaben erlaubte.

205 Vgl. Horster 2001, 14-18.

206 Bei CIL IV 1622 und 2307 bezeichnet Zangemeister den Beschreibstoff als lapis tofaceus. Es handelt sich jedoch um einen deutlich härteren Lavastein.

207 Vgl. Fiorelli 1867, 37-39. Vetter 1953, 46-54. Travertin: Ve 8. 12. 13. 16. 17. 20. 21. 22; Marmor: Ve 9. 14. 107; Tuff: Ve 22c; „Stein“: Ve 10. 22a. 22b. 22d sowie Rix Po 15-18. Die Angaben unterscheiden sich allerdings bei Ve 11 (Fiorelli: Marmor, Vetter: Travertin), Ve 15 (Fiorelli: Tuff, Vetter: Travertin), Ve 19 (Fiorelli: Marmor, Vetter: Travertin).

208 Es handelt sich um die einzige bisher bekannte oskische Steininschrift aus Herculaneum. Das Verb prúffed kann hier nicht die gleiche Bedeutung haben, wie etwa prúffated in Bauinschriften, das sich meist darauf bezieht, dass am Ende der Bauzeit die zuständigen Beamten eine fertige Arbeit abnahmen. Stattdessen handelt es sich hier um eine Weihung an die Göttin Herentas Herukina. Vgl. Vetter 1953, 49 und insbesondere Antonini 1978, 873. Johann Zvetaieff dagegen hält prúffed für die Entsprechung von probavit: Zvetaieff 1886, 145. Ebenfalls kritisch: von Planta 1897, 616-617. Vgl. zu der Inschrift: Scotto di Freca 1993, 322; 323 Abb.1 d; Taf. 56 c (um 180 gedreht). Die Form Herentas ist im Oskischen nicht belegt aber in einer pälignischen Inschrift: Ve 213. Vgl. dazu: von Planta 1897, 70. 209 Mau 1908, 76; Sampaolo 1997, 301. 


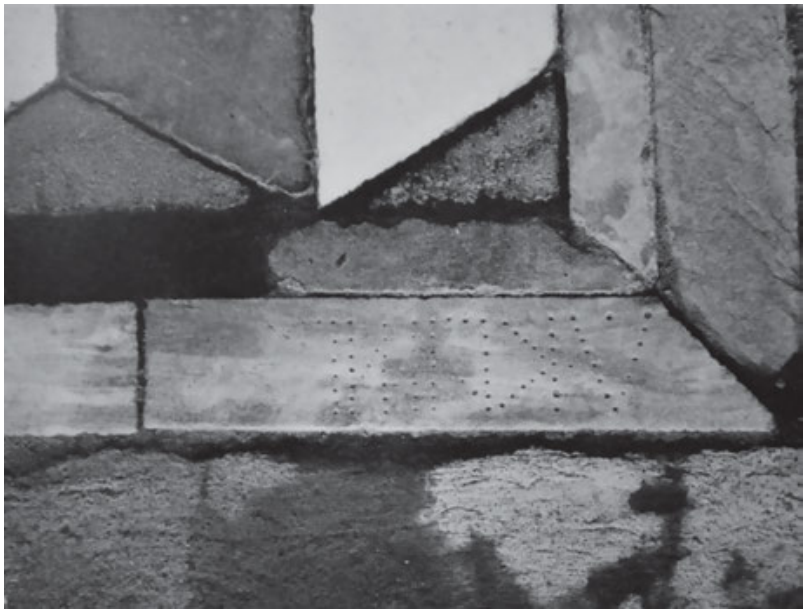

Abb. 3: Ausschnitt aus der Stifterinschrift im Fußboden der cella des Apollontempels in Pompeji. (CICCD - Gabinetto Fotografico Nazionale, Fondo GFN, Inv. N051129.

\subsubsection{Schriftarten und Gestaltung}

Hinsichtlich der Schriftarten und der Gestaltung stellt sich über die reine Benennung der Schriftform die Frage, wie diese ausgeführt wurde und ob sich aus dem Material selbst Hinweise auf den Vorgang der Planung und Anbringung ergeben. Neben den Buchstabenformen sind daher vor allem Farbreste und Werkzeugspuren relevant. Als Schriftarten kommen bei den Steininschriften zuallermeist die capitalis monumentalis oder die capitalis actuaria vor. ${ }^{210}$ Beide Schriftarten wurden in den steinernen Untergrund eingemeißelt, wobei die einzelnen Linien in der Regel einen V-förmigen Querschnitt aufweisen, den Keilschnitt. Sehr wahrscheinlich waren die meisten der Inschriften farbig gefasst. ${ }^{211}$ CIL X 792 (Abb.F8) bietet hierfür ein deutliches Beispiel. Über den konkreten Arbeitsablauf geben manche Inschriften selbst Auskunft. Grundlegend hat sich mit diesem Thema Jean Mallon befasst, der die Hilfslinien, die zur ordinatio, der Anordnung des Textes, dienten, untersuchte. ${ }^{212}$ Insbesondere die Untersuchungen von Catich und Richard D. Grasby berücksichtigen außerdem die Rolle des Farbauftrags nicht nur zum Ausmalen der Inschriften, sondern bei der ordinatio. Sie nehmen an, dass die zu meißelnden Inschriften zunächst nach einem geometrischen Raster geplant wurden, dass man danach die Buchstaben mit einem flachen Pinsel malte, und dass erst danach, also die Formen der gemalten Buchstaben befolgend, die Linien gemeißelt wurden. ${ }^{213}$

210 Vgl. Petrucci 1992, 51-52; Koch 2007, 37.

211 Donati 1998, 98.

212 Mallon 1955. Studien zur ordinatio auf pompejanischem Material: Sertà 1996 (Grab der Mammia); Sertà 1998 (Stele des Titus Suedius Clemens vor der Porta Ercolano); Sertà 2002 (Stele des Titus Suedius Clemens vor Porta Marina und Porta Nocera).

213 Catich grundlegend zur Bedeutung des Pinsels bei der Gestaltung der römischen Inschriftenschrift: Catich 1968, passim. Grasby 2002, 164-168. 
Bei einigen Inschriften sind Hilfslinien zu erkennen, die darauf hindeuten, wie die Inschrift geplant wurde. ${ }^{214}$ Meist achteten die Handwerker jedoch darauf, die Spuren der Herstellung zu minimieren. Es ist davon auszugehen, dass alle Inschriften dieser Art nicht nur gemeißelt, sondern auch ausgemalt wurden. So konnte die Inschrift ihre Wirkung sowohl durch die gesteuerte Verschattung als auch durch den farblichen Kontrast zwischen weißem Stein und meist roter oder schwarzer Farbe erzielen. ${ }^{215}$ Außer den Farbspuren findet sich als weitere Technik das Einlegen von Metalllettern in eine dafür präparierte Steinfläche. Die Metalllettern fehlen heute meist, doch die gemeißelten Dübellöcher bieten deutliche Belege. Diese Technik wurde in Pompeji unter den Ehreninschriften allein bei dem Monument mit zugehöriger Inschrift für Marcus Holconius Rufus in der Sitzstufe des Großen Theaters in Pompeji angewendet (Abb. F13). Auch bei der Stifterinschrift, die die Neupflasterung des forum in Erinnerung halten sollte, zeugen die Vertiefungen von ehemals eingelegten Lettern. ${ }^{216}$ Bei dieser Art von Inschriften handelt es sich um eine besonders aufwendige und sicherlich auch kostspielige Variante, die an sich bereits eine Auszeichnung dargestellt haben dürfte. Dieser hohen Auszeichnung ging auf Seiten der Stifter die Bereitschaft voraus, sich für das Gemeinwesen in außergewöhnlichem Maße einzusetzen und auf verschiedene Arten einzubringen.

Bei den Ehreninschriften in Pompeji und Herculaneum kommt ausschließlich die capitalis monumentalis oder quadrata vor. Bau- und Stifterinschriften wurden in der Regel ebenfalls in capitalis monumentalis verfasst. In den auch $79 \mathrm{n}$. Chr. noch erhaltenen älteren Exemplaren begegnete aber auch die serifenlose Schrift, die bis ins 1. Jh. v. Chr. gebräuchlich war. Die meisten Grabinschriften sind in einer rechtwinklig konstruierten und meist sehr sorgfältig gemeißelten monumentalis geschrieben, wie zum Beispiel die Inschrift an EN 4 in der Nekropole vor der Porta Nocera (Abb. F14). Nur bei wenigen Exemplaren sind die Buchstaben in den gelängten und stärker geschwungenen Formen der actuaria ausgeführt. Die Lava-cippi sowie die Inschrift des Grabes OS 31 sind dagegen mit einer gänzlich serifenlosen und altertümlich wirkenden Schrift geschrieben. Die lapidarii bedienten sich bei den Grabinschriften grundsätzlich derselben Gestaltungsmuster wie bei anderen Inschriftentypen. Vor der Porta Vesuvio jedoch sind alle drei noch erhaltenen der ursprünglich vier gefundenen Grabinschriften in actuaria geschrieben (Abb. F15. F16. F17). Die Inschriften erscheinen alle drei sorgfältig geplant und äußerst sauber ausgeführt, sodass auch hier ein hoher Grad an Professionalität und Können zu erkennen ist. Das Schreiben einer Inschrift in actuaria war technisch nicht weniger anspruchsvoll und aufwendig als in monumentalis,

214 Zum Beispiel am Schaft der Herme des Norbanus Sorex: Lahusen/Formigli 2007, 126 Abb. 3.

215 Nach Catichs Auffassung dienten die eingemeißelten Furchen wie auch Profilleisten oberhalb von Inschriftenfeldern sogar vorrangig dazu, die Farbe vor dem Ausgewaschenwerden und Verbleichen zu schützen: Catich 1968, 65.

216 Sogliano 1925, 252 Abb. 5. 
sodass es sich nicht per se um eine günstigere Variante gehandelt haben kann. ${ }^{217}$ Daher ist es wahrscheinlich, dass sowohl der persönliche Geschmack als auch der Vergleich mit benachbarten Monumenten, in die ein neu hinzukommender Bau sich einreihte, die Gestaltung der tituli beeinflussten. Ein weiterer Faktor für die Wahl der actuaria könnten deren gelängte Formen sein, die es ermöglichten, bei gleicher Zeilenhöhe mehr Text unterzubringen, als es mit dem quadratischen modulus der monumentalis möglich war. ${ }^{218}$ Für einen ungeübten Leser kann es hingegen schwierig sein, bei der actuaria zwischen $<\mathrm{I}>$ und $<\mathrm{L}>$ zu unterscheiden, da der Arm des $<\mathrm{L}>$ oft von gleicher Länge ist wie eine Serife des $<\mathrm{I}>$, wie in der Grabinschrift des Veius Marcellus (Abb. F17). Gerade bei Namen, die auf <ius > oder <lus > enden, entstehen dadurch mögliche Fehlerquellen. Bei der monumentalis sind die Buchstabenformen hingegen eindeutig, sodass Missverständnisse minimiert werden können. Neben Konventionen spielten demnach praktische Überlegungen mit Blick auf die Textverteilung aber auch auf einen möglichen Leser eine Rolle.

\subsubsection{Zwischenergebnis}

Besonders die drei größten Gruppen der Stein- und Metallinschriften, die Grabinschriften, Ehreninschriften und Stifter-, Bau- und Weihinschriften, weisen Ähnlichkeiten bei der Materialwahl und bei Gestaltungsmustern auf. So wurden die meisten dieser Texte in hellen Marmor oder Kalkstein geschrieben und zwar in einer sehr gut lesbaren capitalis monumentalis. Dies gilt vor allem für die Inschriften, die zur Zeit des Vesuvausbruches noch nicht sehr alt waren und auch deshalb heute zahlreicher erhalten sind. Die meisten dieser Schriftzeugnisse standen nicht für sich allein, sondern waren Teil eines größeren Monumentes oder Bauwerks. Nicht nur die Textinhalte, die für die damaligen Zeitgenossen sicherlich leicht zeitlich einzuordnen waren, sondern auch die Gestaltung und Materialien der Gesamtmonumente und Inschriften waren bereits in der Antike Zeugnisse einer Jahrzehnte oder Jahrhunderte zurückliegenden Vergangenheit, insofern als auch Materialien und Schriftformen auf nicht mehr übliche Gepflogenheiten verwiesen. Die serifenlose Schrift der Bau- und Stifterinschriften zeigt nicht, dass man hierfür altmodische Schriftarten wählte, sondern, dass diese besonders langlebig waren. Die Ehreninschriften, die leichter abzutransportieren waren, waren dagegen einer größeren Fluktuation unterworfen. Die langfristige Haltbarkeit spielte bei der Verwendung von Stein als Beschreibmaterial eine wichtige Rolle. Dies wird in der Beständigkeit der verwendeten Steinsorten und

217 Die entsprechenden Gräber sind innerhalb eines Zeitraumes von immerhin 50 Jahren entstanden. Es handelt sich um die Gräber des Vestorius Priscus, des Marcus Veius Marcellus und der Septumia vor der Porta Vesuvio (vgl. Spano 1910, 402-407; Stefani 1998b, 43; Cicirelli 1998, 45 Anm. 2. 46). Außerdem die Gräber Süd 20 und Nord 2 in der Nekropole vor der Porta Ercolano (vgl. Kockel 1983, 98. 115).

218 So auch: Koch 2007, 35-37. 
der festen Verankerung des Textträgers in einem Monument oder im Boden sowie in der Irreversibilität des Schreibprozesses sowie der Sichtbarkeit von Veränderungen deutlich. Bei den einzelnen Textsorten sind jedoch auch Unterschiede hinsichtlich der konventionellen Verwendung von Materialien und Gestaltungsmustern auszumachen. Diese sind bei näherer Betrachtung nicht als arbiträre Verknüpfung, sondern im Kontext der sozialen Einbettung der Inschriften zu verstehen: Planer, Auftraggeber und Financiers setzten unterschiedliche Prioritäten und mussten zugleich den Erwartungshaltungen der Leser begegnen.

Die Grabinschriften waren Teil des Grabmals und als solche in der Regel direkt vom Grabinhaber finanziert und konzipiert. Somit waren sie Teil der Botschaft, die der Grabinhaber oder -erbauer über sich mitteilen wollte. ${ }^{219}$ Durch die Wahl der Materialien für die Inschrift und das ganze Monument, die oft optisch eine homogene Erscheinung hervorrufen, wurde der titulus physisch integriert. Bei der Gestaltung des Monuments spielten die finanziellen Möglichkeiten, der Vergleich mit benachbarten Monumenten, individuelle Inhalte, sowie das Spektrum an in den Städten üblichen oder möglichen Grabtypen eine Rolle. Diese konventionellen und finanziellen Grenzen brachten wiederum für die Inschrift eine Auswahl an Materialien, Anbringungsmöglichkeiten, Schriftarten und Textlängen mit sich, die insgesamt jedoch vor allem vom Ausdruckswillen des Einzelnen gesteuert waren. Bei den Ehreninschriften ist dagegen eine starke Vereinheitlichung bezüglich der genannten Aspekte zu beobachten. Diese muss dadurch zu erklären sein, dass die zu den öffentlich genehmigten, in Auftrag gegebenen oder sogar finanzierten Monumenten gehörenden Inschriften ebenfalls der Regulierung durch das Gemeinwesen unterlagen. Bei den Bau- und Stifterinschriften verhält es sich wiederum anders. Diese war grundsätzlich auf die Initiative und das Engagement einzelner zurückzuführen. Dennoch haben die entsprechenden Inschriften einen stärker dokumentarischen Charakter. Daher mussten die Verständlichkeit und die Akzeptanz auch durch das Einhalten der Konventionen hinsichtlich Anbringungsstelle, Verhältnis zum gestifteten oder gebauten Objekt und Gestaltung mit Rücksicht auf Wahrnehmungs- und Sehgewohnheiten gewährleistet werden.

219 So auch Cormack 2007, 599. 


\subsubsection{Dipinti}

\subsubsection{Textträger}

Dipinti wurden mit roter oder schwarzer Farbe auf die Außenflächen von Häusern, Tabernen, öffentlichen Gebäuden im ganzen Stadtgebiet sowie auf Grabmälern aufgebracht. ${ }^{220}$ Als Beschreibflächen für die gemalten Wandinschriften lassen sich in Pompeji Ziegelmauerwerk, Stein und vor allem Putz fassen. Hinzu kommen wahrscheinlich Holz- und Steintafeln. ${ }^{221}$

Putz war der häufigste Beschreibstoff. ${ }^{222}$ Die Farben des Putzes und der Anstriche prägten das optische Erscheinungsbild der Stadt in hohem Maße. Rot und Schwarz waren die vorherrschenden Farben. ${ }^{223}$ Im Außenbereich, wo er der Witterung

$220 \mathrm{Zu}$ den Beschreibflächen liegen außerhalb des CIL bisher keine ausführlichen Überlegungen vor. Knappe Angaben finden sich bei Fridell Anter 2011, 263; Mouritsen 1988, 31; Sabbatini Tumolesi 1980, 113. Nur wenige Dipinti wurden in Innenräumen gefunden: so etwa die programmata in der Casa di Iulius Polybius (IX 13, 2), oder die Schriftzüge in I 7, 16, die wie Schreibübungen wirken, da immer wieder Wörter nicht vollständig ausgeschrieben oder einzelne Buchstaben wiederholt wurden (Abb. 56). Zur Casa di Iulius Polybius: Giordano 1974, 27-28; In I 7, 16 fanden sich CIL IV 7243-7249, vgl. die Anmerkung Della Cortes; CIL IV 10229b ist in die Laibung des Gewölbes von Grabmal EN 12 der Nekropole an der Porta Nocera gemalt: Varone/Stefani 2009, 490.

$221 \mathrm{Zu} \mathrm{Holz} \mathrm{als} \mathrm{Träger} \mathrm{von} \mathrm{Inschriften} \mathrm{vgl.} \mathrm{Eck} \mathrm{1998,} \mathrm{205-202.} \mathrm{211-217.}$

222 Vgl. dazu Zangemeisters Kommentar: CIL IV Hauptband S. 8: Tituli honorarii, sive candidatorum commendationes per vias propositi sunt et longe maxima ex parte in opere tectorio albo vel ad eam rem in tabellae formam dealbato picti, multo minore numero in tofo dealbato vel in opere latericio dealbato. Mit der Frage nach der Beschaffenheit verschiedener pompejanischer Putze hat sich Walter Klinkert bereits 1957 befasst: Klinkert 1957, 129. Informationen dazu finden sich auch im Rahmen von Beschreibungen einzelner Häuser, vor allem in der Reihe Häuser in Pompeji: Strocka 1984, 18; Ehrhardt 1988, 14; Michel 1990, 16-18; Strocka 1991, 18; Seiler 1992, 20; Stemmer 1992, 15; Staub Gierow 1994, 19-20. 47-48; Fröhlich 1996, 15; Ehrhardt 1998, 21. 24; Staub Gierow 2000, 86; Ehrhardt 2004, 29; Peters 1993, 43. 50; Ling 1997, 258-321 jeweils im Rahmen der Beschreibung der Außenwände der einzelnen Häuser. Die Typologie der Putze, die in den dort behandelten Häusern vorkommen, wurde von Reinhard Meyer-Graft erstellt: Meyer-Graft/Ehrhardt 1997. Eine breite Materialbasis zu den Putzen stellt auch Agneta Freccero vor, allerdings vor allem zu Innenräumen: Freccero 2005; Freccero 2007; Freccero 2012.

223 Spinazzola hat das Farbspektrum der verputzten Fassaden beschrieben, das sich in Folge der Grabungen im Bereich der Via dell'Abbondanza präsentierte: Spinazzola 1953, 245-246. Fridell Anter ergänzt dessen Beschreibungen und Rekonstruktionen durch eigene Beobachtungen sowie weitere Hinweise wie das Korkmodell im Museo Archeologico Nazionale di Napoli: Fridell Anter 2011, 252-260. Gerade die Farben, die für die Bemalung der Wände in Pompeji verwendet wurden, waren bereits Gegenstand mehrerer Untersuchungen. Grundlegend zu den in Pompeji verwendeten Farben ist Augusti 1967. Selim Augusti befasst sich sowohl mit den verschiedenen Pigmenten, vor allem aber auch mit der Terminologie in der zeitgenössischen Literatur, wobei vor allem an Vitruv und Plinius den Älteren zu denken ist; den archäologischen Befund anhand von Proben aus zwei Häusern in Pompeji (Casa die Pittori al Lavoro (IX 12, 9) und Casa di Fabius Rufus (VII 16, 17-22)) haben Antonio Varone und Hamdallah Béarat untersucht und vorgestellt: Béarat/Varone 1997. Zu ästhetischen Aspekten der Farbigkeit des öffentlichen Raumes in jüngerer Zeit vor allem: Fridell Anter 2011, insbesondere 291-295. 
ausgesetzt war, wurde vornehmlich der lange haltbare Cocciopesto-Putz verwendet. Sein hellroter Farbton ergab sich dadurch, dass zerstoßene Ziegel als hydraulischer Zuschlag beigemengt wurden. Dadurch wurde der Putz wasserfest. Auch Vitruv empfiehlt einen solchen Spezialputz für Flächen, die in erhöhtem Maße Feuchtigkeit ausgesetzt waren. ${ }^{224}$ In vielen Fällen wurden die entsprechenden Fassaden nicht weiter gestrichen und waren daher als hellrote Fläche zu sehen. ${ }^{225}$

Abgesehen davon waren Rottöne, Schwarz, Weiß, Gelb sowie grünbraune Töne im Straßenbild vorherrschend. ${ }^{226}$ Karin Fridell Anter kann feststellen, dass bei einer Mehrheit der Gebäude (75\%) die Fassade im Bereich des Erdgeschosses auf einer Höhe von 150 bis $300 \mathrm{~cm}$ horizontal unterteilt war. Die obere Zone ist in den allermeisten Fällen der Farbe des normalen Kalkputzes entsprechend hell. Die untere Zone dagegen war meist rot (70\%), gelb, schwarz oder unbemalt (Abb. F18). Zudem konnte die untere Zone (in ca. $20 \%$ der Fälle) durch aufgemalte Linien als Orthostatenzone charakterisiert werden. Falls die obere Zone durch Bemalung gestaltet war, zeigte diese meist figürliche Darstellungen wie Gottheiten, Heroen oder Schreine. ${ }^{227}$ Dabei lässt sich immer wieder beobachten, dass nicht der Wandabschnitt die Einheit bildete, die durchgehend gestaltet wurde, sondern dass vielmehr genau auf der Höhe der Zwischenwände zwischen den Häusern oder Grundstücken ein Wechsel festzustellen ist, wie zwischen den Eingängen I 12, 3 und 4 (Abb.F19). Die einheitliche Gestaltung der Fassade eines zusammengehörenden Besitzes hatte sehr wahrscheinlich unter anderem die Funktion, dessen Geschlossenheit zur Schau zu stellen, wie Felix Pirson überzeugend am Beispiel der Casa del Menandro dargelegt hat. ${ }^{228}$

Die Zusammensetzung der Farben spielt dort kaum eine Rolle. Als Grundlage dienten Fridell Anter besonders die Beschreibungen und Rekonstruktionen von Spinazzola im Bericht über die Nuovi Scavi di Via dell'Abbondanza: Spinazzola 1953, 242-252.

224 Vgl. Vitr. 7,4,1: Et primum conclavibus, quae plano pede fuerint, in imo pavimento alte circiter pedibus tribus pro harenato testa trullisetur et dirigatur, uti eae partes tectorium ab umore ne vitientur. Vgl. Allag/Barbet 1972, 967-969.

225 Fridell Anter 2011, 267. Vgl. auch Allag/Barbet 1972, 967.

226 Fridell Anter 2011, 269.

227 Fridell Anter 2011, 274-276. Dabei wird auch deutlich, dass schwarze Zonen immer in Orthostaten unterteilt waren, rote Flächen sowohl unterteilt als auch monochrom vorkommen und gelbe Flächen tendenziell als Marmorimitation gestaltet war. Fridell Anter geht davon aus, dass für diese Bemalungen nahezu ausschließlich Kalkfarben in Frescotechnik verwendet wurden: Fridell Anter 2011, 267268. Laut Thomas Fröhlich fanden sich derartige Götterbilder vor allem an Fassaden von Läden und Kneipen: Fröhlich 1991, 48. Zu dem spannungsreichen Verhältnis zwischen figürlichen Malereien und Wandinschriften vgl. auch Kellum 1999, 290-291.

228 Pirson 1999, 63-64. Pirson betont zu Recht, dass die farblichen Fassungen auch aufgrund ihrer relativ leichten Veränderbarkeit eine wichtige Quelle für die Rekonstruktion von Besitzverhältnissen und deren Verschiebungen bieten können, sofern sie erhalten sind. 
Auffällig ist auch, dass der Putz in der unteren Zone häufig feiner und dichter ist, als in der darüber liegenden Zone. ${ }^{229}$ Dabei ist die horizontale Grenze zwischen den Putzqualitäten meist deutlich zu erkennen. Diese Unterteilung der Wand hängt möglicherweise mit den Vordächern zusammen, die an vielen Häusern belegt sind. ${ }^{230}$ Im unteren Bereich der Fassade war die Wand stärker Niederschlägen und Spritzwasser von der Straße ausgesetzt, während die höheren Bereiche eher von den Vordächern geschützt wurden. Die Kompaktheit und die Geschlossenheit der Oberfläche, die sowohl durch den hydraulischen Zuschlag als auch durch Versinterung der freskal aufgebrachten Farben zustande kam, hatten demnach auch den praktischen Nutzen, die Wand noch stärker zu schützen, als dies ein weniger verdichteter oder unbemalter Putz vermochte.

Dass die Beschaffenheit des Putzes sehr wohl Einfluss auf das Aussehen der Aufschriften hatte, ist an CIL IV 7993 auf der Wand zwischen III 2, 1 und III 2, 2 zu erkennen, wo an der Grenze zwischen den beiden Einheiten auch die Verkleidung der Wände wechselt (Abb. 40). ${ }^{231}$ Für die Beschriftung mussten die Wandflächen vorbereitet werden. Wenn die untere Zone dunkel bemalt war und dort ein Dipinto angebracht werden sollte, tünchte man die für die Aufschrift benötigte Fläche weiß, damit die meist schwarze oder rote Schrift überhaupt zu sehen war. Auch helle Putzflächen wurden häufig auf diese Weise vorbereitet. An einem Dipinto (CIL IV 7241) an der O-Seite der südöstlichen Ecke von insula I 7 (Abb. F20) ist deutlich zu erkennen, dass die rot gemalten Buchstaben im Bereich der weißen Fläche schärfer umrissen sind als dort, wo sie darüber hinausragen. Die weiße Farbe bestand aus Kalkmilch und konnte so die Rolle eines Vermittlers zwischen dem Putzuntergrund und der kalkbasierten Farbe erfüllen, die dadurch wiederum al fresco aufgebracht wurde. ${ }^{232}$ Dass die rote Farbe

229 Ein Gegenbeispiel stellt die Fassade der Casa dei Cei (I 6, 15) dar, deren obere Zone mit Stuck als isodomes Mauerwerk gestaltet ist. Diese Gestaltungsweise ist jedoch in Pompeji selten. Vgl. Fridell Anter 2011, 276.

230 So zum Beispiel an der Casa di Trebius Valens (III 2, 1): Spinazzola 1953, 281; Casa di Paquius Proculus (I 7, 1): Spinazzola 1953, 297; Casa di Marcus Lucretius Fronto (V 4, a): Peters 1993, 41.

231 Vgl. Varone/Stefani 2009, 239. An den Fassaden wurde mit Kalkputzen unterschiedlicher Qualität gearbeitet: Klinkert 1957, 114. 129. Vgl. auch: Shaer 2005, 23-25. Meyer-Graft stellt die in Pompeji verwendeten Putze am Beispiel der Casa delle Nozze d'Argento (V 2, i) in eine chronologische Abfolge: Meyer-Graft/Ehrhardt 1997, 318. Beschreibung der Typen: 320-322. Zu ähnlichen Ergebnissen wie Meyer-Graft kommt auch Freccero: Freccero 2012, 89-93. Die von Meyer-Graft vorgestellten Proben stammen jedoch alle aus Innenräumen. Die Beschreibungen der Fassadenputze in der Reihe Häuser in Pompeji bieten weitere Anhaltspunkte zur Chronologie und Beschaffenheit der Materialien. So kann festgehalten werden, dass die vorgefundenen Putze teilweise wasserfest waren und dass es keine Putze gab, die nur im Außen- oder nur im Innenbereich zum Einsatz kamen: Ehrhardt 1998, 21. 24. 165; Seiler 1992, 20; an der Fassade der Casa dell'Ara Massima haben sich Reste eines Putzes erhalten, der Pozzolanerde und Ziegelsplit enthielt, und demnach gezielt als wasserfester Putz dort verwendet worden sein muss: Seiler 1992, 15. 141; Ehrhardt 1988, 84 Abb. 42. Auch andernorts waren Ziegelsplit oder andere zerkleinerte Terracottazuschläge üblich, vgl.: Siddall 2006, 209.

232 Ettl/Hundbiß 2011, 48. 
dabei in die noch nasse Kalkmilch gestrichen wurde, ist an Putzresten an den Außenwänden der praedia Iuliae Felicis (II 4) und an der NO-Ecke der insula IX 5 zu erkennen (Abb. F21). Die Gestaltung dieser Flächen, die mitunter in der Form der tabula ansata gestaltet waren, wird an späterer Stelle behandelt werden. ${ }^{233}$

Die noch erhaltenen Fassaden der Gräber vor den Stadttoren waren zu großen Teilen ebenfalls verputzt oder mit Tuff verkleidet und wurden intensiv als Beschreibflächen genutzt. Dies ist insbesondere für die Nekropole an der Porta di Nocera gut dokumentiert. ${ }^{234}$ Ebenso ist anzunehmen, dass auch die Fassaden der Gräber in den anderen Nekropolen als Beschreibflächen dienten, auch wenn im Rahmen der Ausgrabungen nur wenige Dipinti registriert wurden oder davon heute kaum noch Spuren zu erkennen sind. ${ }^{235}$ Der Putz an den Gräbern ist meist sehr hell und bietet somit sehr gute Voraussetzungen für die Anbringung von Dipinti. ${ }^{236}$ Auch Stein konnte als Beschreibfläche für gemalte Aufschriften dienen. Besonders im Westen der Stadt bestanden Teile der sichtbaren Fassaden aus Sarno-Kalkstein oder NoceraTuff (Abb. F22). ${ }^{237}$ Nocera-Tuff kann rötlich aber auch gelblich-hellgrau bis grau sein, sodass der Kontrast zur roten oder schwarzen Farbe unterschiedlich hoch war.

Wandinschriften auf Ziegelmauerwerk finden sich in Pompeji ebenfalls. ${ }^{238}$ Als Schreibuntergrund waren die Ziegelflächen aufgrund der Struktur des Mauerwerks deutlich unebener als Putz und die meisten Steinfassaden. Hinzu kommt, dass die rote Malfarbe nur einen schwachen Kontrast zum ebenfalls roten Ziegeluntergrund erzielte, sodass die benötigte Fläche vorher mit Kalkmilch geweißelt wurde. ${ }^{239}$

233 Vgl. Kap. 2.2.3.1, S. 69.

234 Vgl. CIL IV 9936-9986.

235 Vgl. das Grab des Marcus Obellius Firmus vor der Porta di Nola oder das Grab Nord 38 in der Nekropole vor der Porta Ercolano. Zur Nekropole vor der Porta di Nola: De Caro 1979, 72-79; zur Nekropole an der Porta Ercolano: CIL IV 84-89 und Kockel 1983, 174; zu den Gräbern im sogenannten Fondo Pacifico, die eine Fortsetzung der Gräberstraße vor der Porta Nocera bilden: CIL IV 3857-3879; zu den Gräbern von der Porta Vesuvio: CIL IV 7976-7981.

236 Vgl. Kockel 1983, 86. 91. 99. 174. 178. 181; D’Ambrosio/De Caro 1983 (ohne Seitenzahlen) zu den Gräbern EN 4, 6, 10, 12, 14, 20, 22, 30, 36, 38, 42, und 44; ES 5, 7, 9, 11, 13, 19; OS 1, 3, 5, 7, 9, 11, 13, 27 und 29.

237 Vgl. Fridell Anter 2011, 263; Kawamoto/Tatsumi 1992, 95-97; Mau 1908, 34. Innerhalb der zugänglichen Gebiete der Stadt finden sich Tuffpfeiler östlich der Via Stabiana nur an der Südseite von insula $\mathrm{V} 1$.

238 Insbesondere an der Via Stabiana, die von Tabernen gesäumt ist, finden sich Reste von weißer und roter Farbe auf den Pfeilern zu den Seiten der Eingänge. Weitere Beispiele sind an verschiedenen Stellen im Stadtgebiet zu finden, wie zum Beispiel an der NO-Ecke der insula IX 4 oder zwischen den Eingängen VIII 4, 5-7. Vgl. Varone 1987, 91-95. Zu diesem Befund auch Dickmann/Pirson 2002, 255-257 mit Verweis auf den Nachweis weiterer Erdbeben in den 70er Jahren des 1. Jh.s. Die Tatsache, dass auch direkt auf die opus latericium-Fläche gemalt wurde, belegt, dass diese Ziegelflächen nicht immer verputzt, sondern sichtbar waren. Vgl. dazu Fridell Anter 2011, 264.

239 Es konnte nur ein Beispiel gefunden werden, in dem ein Dipinto ohne eine vorherige Weißelung auf das Ziegelmauerwerk aufgebracht wurde: CIL IV 9830, rechts von Eingang I 9, 11. Vgl Varone/ Stefani 2009, 114. Taf. 9 Mitte. 
Ein weiterer Beschreibstoff für Dipinti war Holz. Tabulae dealbatae oder alba auf mobilen Textträgern wurden in Pompeji jedoch bisher nur in fragmentarischer Form und meist ohne gesicherten Kontext gefunden. ${ }^{240}$ Ein Reflex dieser Praxis hat sich auf dem Wandgemälde aus dem atrium der praedia Iuliae Felicis (II 4, 3) erhalten, wo mehrere Personen zu sehen sind, die eine beschriftete und an Statuensockeln befestigte Tafel betrachten. ${ }^{241}$ Diese Tafeln waren vermutlich meist aus Holz und konnten an Bauwerken oder Denkmälern angebracht werden. In den Texten, die solche Tafeln erwähnen, wird auch darauf Bezug genommen, dass sie mit schwarzer Farbe - atramento - beschrieben wurden. ${ }^{242}$

\subsubsection{Verwendete Materialien und Werkzeuge}

Das Spektrum der verwendeten Farben umfasst Weiß-, Schwarz- und Rottöne, ${ }^{243}$ also Töne, die auch in der Bemalung der Fassaden häufig vertreten waren. ${ }^{244}$ Bei weitem am häufigsten kommt Rot als Schreibfarbe für programmata, edicta munerum und andere Arten von Dipinti vor. ${ }^{245}$ Schwarz findet ebenfalls regelmäßige Verwendung in allen inhaltlichen Gattungen. Rot und Schwarz wurden teilweise nebeneinander, in zwei aufeinander bezogenen Inschriften CIL IV 7242 (Abb. F23), oder sogar innerhalb derselben Inschrift verwendet, wie in CIL IV 7991 (Abb.F24). Weiß wurde fast ausschließlich dazu verwendet, die benötigte Fläche zu grundieren und für die Dipinti vorzubereiten, wie an zahlreichen Beispielen zu erkennen ist (Abb.F24). Zusätzlich kommt es in einigen Inschriften vor. Zwar ist kein Beispiel eines weiß geschriebenen edictum muneris bekannt, wohl aber sind programmata und Aufschriften anderen Inhaltes belegt (Abb.4). Darunter sind sogenannte cacator-Inschriften, aber auch solche, die sich inhaltlich keiner größeren Gruppe zuweisen lassen. ${ }^{246}$

Was die Beschaffung, die Kosten und Verarbeitung der Farbstoffe betrifft, sind ebenfalls Unterschiede festzustellen. Wiederum stellt sich das Problem, dass in den Schriftquellen keine Aussagen zu finden sind, die dezidiert Pigmente und Bindemittel

240 Vgl. CIL X 884-923. Bei den in der Casa del Menandro gefunden Marmorfragmenten handelt es sich sicher nicht um alba, da sie diesen in Gestaltung und Inhalt nicht entsprechen. Vgl. Allison 2006, 71 Nr. 247 und 248.

241 Vgl. Kap. 3.3.2, S.152; Nappo 1989, 88. 87 Abb.10.

242 Lex Acilia repetundarum (CIL I 583) 14. Pagano interpretiert mehrere Fragmente einer marmornen Tafel aus Herculaneum als Reste eines album: Pagano 1992, 189-191. Es handelt sich dabei um eine Namensliste, die mit schwarzer Farbe auf den hellen Untergrund gemalt ist. Er nimmt an, dass die Buchstaben in einem zweiten Schritt ausgemeißelt werden sollten (Pagano 1992, 191).

243 Sehr selten kommen auch gelbe gemalte Inschriften vor, wie zum Beispiel: CIL IV 3436 und 3437. 244 Fridell Anter 2011, 276.

245 Vgl. den Hinweis Maus und Zangemeisters in CIL IV Suppl. 2, 468: ubicumque tituli color non indicatur, rubro colore scriptus est.

246 Mit weißer Farbe geschriebene Dipinti: CIL IV 13, 814, 815, 2883 (programma), 2884 (programma), 3359, 3405, 3611-3613 (programmata), 7124, 7125, 7714 (cacator-Inschrift), 7715 (cacator-Inschrift), 7796 (programma). 


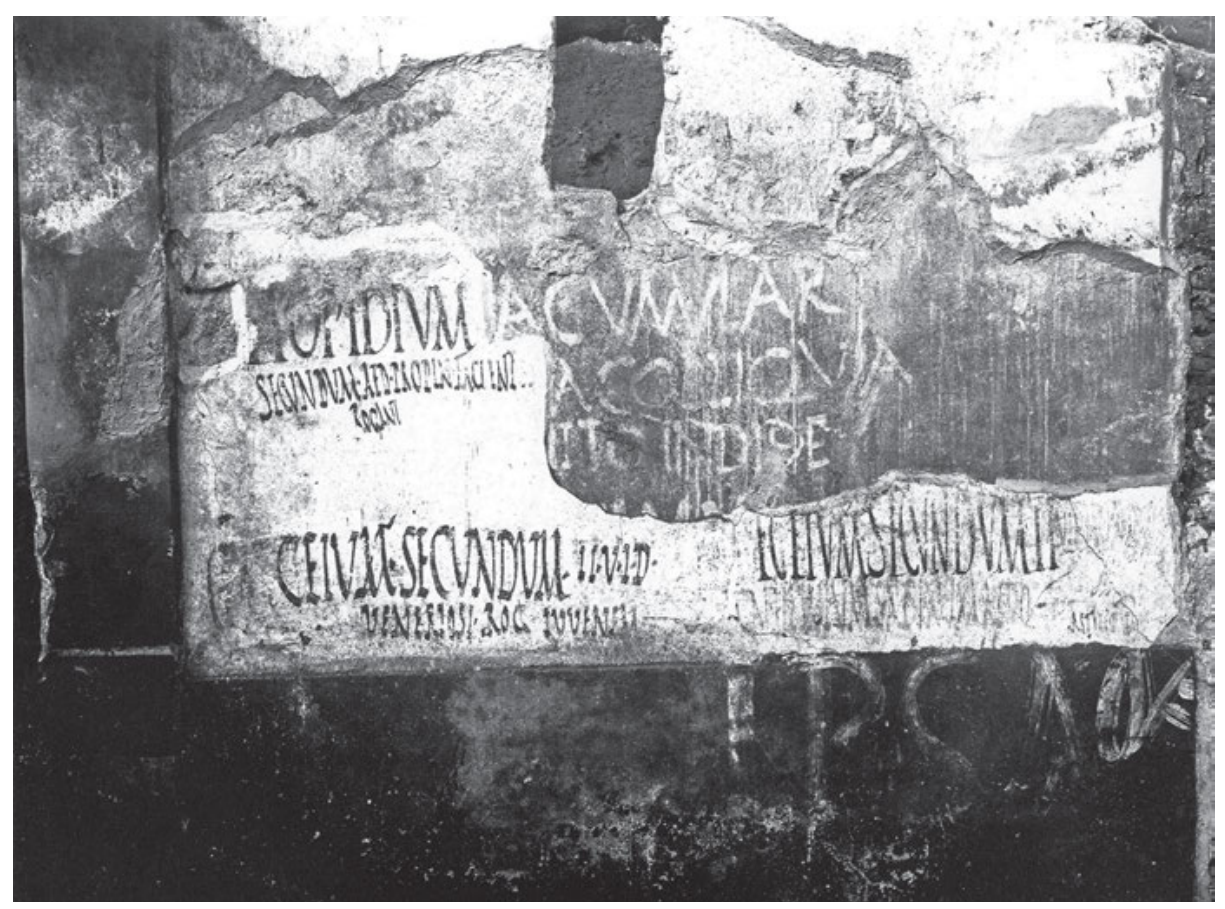

Abb. 4: Mit Kalkmilch geschriebene Dipinti an der Fassade von III 7, 1: CIL IV 7124.7125 und 7796. (c) Su concessione del Ministero per i Beni e le Attività Culturali e per il Turismo - Parco archeologico di Pompei. Jegliche weitere Reproduktion oder Duplikation ist untersagt.

für den Außenbereich betreffen. Abgesehen davon bieten Vitruv und Plinius jedoch wertvolle Informationen. ${ }^{247}$ Beide gehen darauf ein, welche Pigmente es für die verschiedenen Farbtöne gibt, wie diese gewonnen werden, welche Bindemittel dafür in Frage kommen und wie sie aufgetragen werden. ${ }^{248}$

Rubrica ist der Begriff, den sowohl Plinius als auch Vitruv wählen, um roten Ocker zu beschreiben, wobei Vitruv betont, dass dessen Varianten häufig vorkommen und praktisch überall verfügbar sind. ${ }^{249}$ Nach Augusti ist rubrica bei weitem das häufigste rote Pigment. ${ }^{250}$ Rubrica zeichnet sich über diese leichte Verfügbarkeit hinaus auch

247 Exemplarisch seien hier genannt: Augusti 1967, 34. 77-92. 110-113, Béarat 1997, 25-29 sowie Allison 2002, 200-203.

248 Vgl. Vitr. 7,5,8; 7,7,1.2; 7,10,2-4; Plin. nat. 35,31-35; 35,41-43; 35,49.

249 Plin. nat. 35,33-35; Vitr. 7,7,2: item rubricae copiosae multis locis eximuntur. Mit dem Begriff wird tatsächlich nicht nur der Farbton, sondern vor allem das Pigment bezeichnet: Oxford Latin Dictionary (2007) 1664 s. v. rubrica. In den Fällen, in denen das CIL die Farbe der Inschriften nennt, werden Rottöne meist mit colore rubro oder rubrica bezeichnet. Vgl. CIL IV 495. 1177. 1180. 7243.

250 Vitr. 7,5,8. Von insgesamt 51 Proben bei Augusti sind 45 mit rubrica bezeichnet: Augusti 1967, 153-158. Ein ähnliches Bild ergibt sich aus den Fallstudien in Béarat/Varone 1997, 208-210. Zangemeister scheint dagegen anzunehmen, dass sowohl die roten als auch die schwarzen Dipinti mit 
durch eine Reihe chemischer Eigenschaften aus. Insbesondere sind Ocker Laugen-, UV- und witterungsbeständig und in allen Maltechniken anwendbar. ${ }^{251}$ Hauptbestandteil ist immer Hämatit, wobei jedoch deutliche Unterschiede im Farbton vorkommen, die von unterschiedlichen Zusammensetzungen herrühren. ${ }^{252}$ Im Befund sind auch heute noch verschiedene Rottöne zu erkennen (Abb. F20. F25. F46. F62), die von Braun bis zu leuchtendem Rot reichen und sich teilweise auch so überlagern, dass diese Unterschiede im direkten Vergleich ins Auge fallen. ${ }^{253}$

Plinius unterscheidet zwischen mehreren hochgeschätzten rubrica-Sorten, die aus unterschiedlichen Gebieten stammen: Sinope, Ägypten, Balearen, Africa, Lemnos und Kappadokien. Für drei davon nennt er den Preis und typische Verwendungsweisen: pretium optimae X II, usus ad penicillum aut si lignum colorare libeat. eius, quae ex Africa venit, octoni asses, cicerculum appellant; magis ceteris rubet, utilior abacis. Idem pretium et eius, quae pressior vocatur, et est maxime fusca. usus ad bases abacorum (Plin. nat. 35,31-32). Bei diesen drei Sorten unterschied sich der Preis also um den Faktor 4. Da Plinius ebenfalls betont, dass die rubrica lemnia als die beste angesehen wurde, ebenso aber allenthalben verschiedene nicht erwähnenswerte Varianten zu finden waren, ist anzunehmen, dass die Preisspanne noch erheblich größere Abstände aufwies. ${ }^{254}$ Inwieweit diese Preise auch für den Markt in Pompeji galten, ist fraglich. So ist festzuhalten, dass rubricae im Vergleich mit anderen Rottönen deutlich günstiger waren und dass anzunehmen ist, dass abgesehen von importierten, namhaften Sonderarten auch lokale Vorkommen Verwendung fanden. ${ }^{255}$ Gerade an den Abweichungen der Farbtöne zeigt sich, dass die Maler nicht nur eine Sorte nutzten, sondern auf mehrere verfügbare Quellen zurückgriffen.

Schwarz stellt als Farbton die zweite große Gruppe unter den Dipinti. Bei Vitruv und bei Plinius findet sich die Bezeichnung atramentum für eine schwarze Farbe, die zu den künstlichen zählt, da sie durch spezielle Vorgänge eigens hergestellt werden musste. ${ }^{256}$ Der Vorgang, durch den das atramentum gewonnen wird und den die

minium gemalt wurden, wobei die schwarze Färbung erst durch Umwelteinflüsse entstanden sei: CIL IV Hauptband S. 8; Vgl. dazu Vitr. 7,9,1-2. Irreführend: Plin. nat. 33,42; vgl. Donati 1998, 98.

251 Dazu: Wülfert 2013.

252 Béarat 1997, 29.

253 Vgl. dazu auch Della Cortes Beobachtung zu den sich überlagernden Dipinti in der Nekropole an der Porta di Nocera: Della Corte 1958b, 143 Nr. 344 b.

254 Vgl. Plin. nat. 35,32-33.

255 Vgl. die Liste bei Augusti 1967, 147.

256 Im CIL wird dies mit colore nigro angegeben (CIL IV 3375. 3382. 3385. 3497). Vitr. 7,10,1-4; Vitruv verwendet hier den Ausdruck quae ex aliis generibus tractationum temperaturis commutata recipiunt colorum proprietates; Plinius spricht von atramentum als facticios: Plin. nat. 35,41-43. Atramentum bezeichnet in der antiken Literatur anders als rubrica vor allem den Farbton, kann also - sofern nicht näher spezifiziert - auch aus etwas anderem als Ruß hergestellt worden sein: „atramentum“ im Oxford Latin Dictionary (2007), 198. Davon zu unterscheiden ist Tinte, die mit atramentum librarium bezeichnet wird: Graux 1877, 529-530. 
Autoren in diesen Textstellen beschreiben, besteht darin, bestimmte Materialien unter kontrollierten Bedingungen zu verbrennen und den Ruß aufzufangen. Interessant an beiden Passagen ist, dass Vitruv und Plinius jeweils hervorheben, dass es die Möglichkeit gibt, besonders hochwertige schwarze Pigmente aus Pinienholz oder -harz $\left(\right.$ taedis $^{257}$ ) oder anderem Harz (resina) herzustellen. Zudem kamen jedoch auch Substanzen wie Weinhefe (vini faex) zum Einsatz. In Ermangelung dieser mitunter schwierig zu beschaffenden Rohstoffe konnten auch nicht näher spezifizierte sarmenta et taedae verbrannt und entsprechend angerührt werden. Daraus ergibt sich, dass atramentum zwar ein Pigment war, das eigens fabriziert werden musste, das auf der anderen Seiten aber aus einer großen Anzahl an verschiedenen Rohstoffen gewonnen werden konnte. ${ }^{258}$ Weitere Arten von schwarzen Pigmenten wurden zwar in Pompeji nicht gefunden oder jedenfalls nicht identifiziert, waren aber sicherlich in Gebrauch. ${ }^{259}$ Béarat hat außer Ruß auch Holzkohle identifiziert, die anhand kleiner, aber mit dem bloßen Auge sichtbarer Holzstückchen zu erkennen ist. Ruß ist auch unter den von ihm untersuchten Proben das am häufigsten gefundene Pigment. ${ }^{260}$

Weiß kommt zwar als Schreibfarbe vor, hatte jedoch für die Vorbereitung und Grundierung der Beschreibfläche eine viel größere Bedeutung. ${ }^{261}$ Weder Plinius noch Vitruv gehen auf Kalk als Farbe ein, beide nennen und beschreiben jedoch eine Reihe anderer Weißpigmente. ${ }^{262}$ Dennoch ist es nicht verwunderlich, dass Kalk für die Vorbereitung der Beschreibfläche Verwendung fand. Auch für den großflächigeren Anstrich wurde Kalk in Form von Kalkschlämme in Pompeji verwendet. So fanden sich

257 Taeda bezieht sich auf Pinienholz, -harz oder Produkte daraus. Vgl. dazu „taeda“ im Oxford Latin Dictionary (2007), 1900.

258 In diesem Sinne auch Allison 2002, 202-203. Elisabetta Gliozzo nimmt zur Frage, inwieweit Ruß als Pigment kommerzialisiert war, an, dass nur Sonderarten, denen besondere Qualitäten zugesprochen wurden, importiert wurden (Gliozzo 2007, 80.). Zur Gewinnung von Ruß: Augusti 1967, 111 und die Beschreibung bei Vitruv $(7,10,2)$. Hier finden sich Hinweise darauf, dass spezielle Gebäude verwendet wurden, sodass mit einer vorrichtungs- und wahrscheinlich auch personenabhängigen Herstellung zur rechnen ist. Augusti führt nur einen einzigen Fund an schwarzen Pigmenten auf, bei welchem es sich um Ruß handelt: Augusti 1967, 153. Weitere Beispiele bei Béarat/Varone 1997, 208-210. 259 Augusti 1967, 113; insbesondere elephantium, das bei Plin. nat. 35,42 genannt wird.

260 Béarat 1997, 25-26. Bestätigt auch durch Gliozzo 2007, 80. Gliozzo nennt eine Reihe weiterer, mineralischer Pigmente.

261 Im CIL werden weiße Inschriften im Hauptband und im Supplement 2 mit colore albo und im Supplement 3 mit calce beschrieben. Im Hauptband und Supplement 2 sind das zum Beispiel CIL IV 814 und 815 sowie 3611-3613; im Supplement 3 unter einigen anderen CIL IV 7714. 7715 und 7796. Der zum Beschreiben vorbereitete Grund wird mit tectorio dealbato, tofo dealbato, area dealbata oder auch tabella dealbata benannt, z. B. bei CIL IV 7787. 1190. 7644. 7647.

262 An erster Stelle wird jeweils das paraetonium genannt, worauf melinum und cerussa folgen. Dabei geht es vor allem um Herkunft und Herstellungsweisen und weniger um Einsatzgebiete (Vitr. 7,7,3; 7,12,1; Plin. nat. 35,36-37). Zur Beschaffenheit dieser Pigmente vgl. auch Béarat 1997, und Gliozzo 2007, 73-79. Auch im Kontext des Löschens von Kalk erwähnt Vitruv nicht, dass Kalkschlämme oder Kalkmilch auch zum Anstreichen oder als weiße Farbe verwendet wurde (Vitr. 7,2). Augusti 1967, 59 nennt Kalk nicht unter den weißen Farbstoffen. Entsprechend: Duran et al. 2010, 300. 
an mehreren Fassaden entsprechende Spuren. ${ }^{263}$ Die aufgebrachte Kalkschlämme oder in stärker verdünnter Form: Kalkmilch - hatte dabei nicht nur die Funktion einer weißen Farbe, sondern vermittelt aufgrund ihrer chemischen Eigenschaften zwischen der Wandoberfläche und der darauf aufgetragenen Farbe. ${ }^{264}$ Die chemischen Eigenschaften des stark alkalischen Kalkes sowie auch seine keimtötende Wirkung konnten mitunter auch zu einer Konservierung der Putzfassaden beitragen. ${ }^{265}$

Mit Blick auf die Dipinti, die zwar mit den gleichen Pigmenten wie die flächige Wandmalerei, aber - wenn überhaupt - nur ausnahmsweise in den nassen Putz gemalt wurden, ist es angezeigt, noch einmal die Frage nach den verwendeten Bindemitteln aufzugreifen. Bevor die Pigmente mit Wasser oder einem Bindemittel angerührt werden können, müssen sie allerdings zunächst zerkleinert und durch Schlämmung in Wasser oder mithilfe von Stoffen wie Casein, Leim oder Eigelb gereinigt werden. Diese Vorgänge erfordern einen hohen Grad an Zeit und Sorgfalt, haben aber eine erhebliche Auswirkung auf die optische Wirkung des Endproduktes. ${ }^{266}$ Das Zerkleinern der Pigmente und das Schlämmen konnte von kundigen Malern, die dadurch gezielt das je nach Auftrag gewünschte Ergebnis erreichen konnten, durchgeführt werden. Ebenso ist es aber denkbar, dass es spezialisierte Handwerker und Betriebe gab, die diesen Arbeitsschritt routinemäßig durchführten und Pigmente anboten, die nur noch mit den entsprechenden Bindemitteln angerührt werden mussten. ${ }^{267}$

In den Schriftquellen finden sich wiederum nur Hinweise, die die Wandmalerei im Allgemeinen betreffen und das Augenmerk nicht explizit auf den Außenbereich lenken. So beschreibt Vitruv detailliert, wie die Wand für den Farbauftrag vorzubereiten war und dass die Farben in den noch feuchten Putz hineingemalt werden sollten. ${ }^{268}$ Plinius nennt dagegen eine Reihe von Pigmenten, die nicht al fresco verarbeitet werden konnten: purpurissum, indicum, caeruleum, melinum, auripigmentum, appianum, cerussa. ${ }^{269}$ Aus diesen beiden Textstellen kann demnach abgeleitet werden, dass die Fresco-Technik durchaus geläufig war und zumindest von diesen beiden Autoren als die übliche Verfahrensweise dargestellt wurde, indem sie als besonders vorteilhaft beschrieben und indem nicht kompatible Materialien als Ausnahmen ausgezeichnet werden.

263 Z. B. an der Casa del Principe d Napoli (VI 15, 7.8): Strocka 1984, 18; auch an der Casa del Granduca (VII 4, 56): Staub Gierow 1994, 19-20.

264 Vgl. Ettl/Hundbiß 2011, 49; Frössel 2011, 145 (s. v. Kalkmilch).

265 Maier 2007, 203.

266 Dazu Price 2007, 271-272.

267 In diesem Kontext wäre an eine officina pigmentaria zu denken, wie sie möglicherweise in I 8, 15.16 gefunden wurde. Vgl. dazu Della Corte 1926, 329-330 und Castiglione Morelli del Franco/Vitale 1989, 207. Dazu Wallace-Hadrill 1994, 192: „Deserves further study.“ Als Bäckerei gedeutet: Monteix 2010, 163 Anm.76. 164.

268 [parietes] coloribus cum politionibus inductis nitidos expriment splendores. Colores autem, udo tectorio cum diligenter sunt inducti, ideo non remittunt sed sunt perpetuo permanentes: Vitr. 7,3,7.

269 Plin. nat. 35,49. 
Die Möglichkeit, die Inschriften in den nassen Putz zu malen, bestand in aller Regel bei den Dipinti nicht, sodass die Farben entsprechend anders vorbereitet werden mussten. Interessant ist in diesem Zusammenhang auch, dass sowohl Vitruv als auch Plinius betonen, dass atramentum, falls es für die Wandmalerei verwendet werden sollte, immer mit Leim (glutinum) zu vermischen sei. ${ }^{270}$ Die Kombination des Rußes mit Leim schließt jedoch nicht aus, dass die so gewonnene Mischung mit Wasser verdünnt auch freskal aufgebracht wurde. ${ }^{271}$ In Bezug auf rubrica geben die Autoren keine besonderen Bindemittel an. Allerdings ist roter Ocker mit allen Bindemitteln kompatibel, zeichnet sich durch eine hohe Farbechtheit aus und ist gegenüber Alkalien unempfindlich, weshalb er besonders gut in der Fresco-Technik eingesetzt werden kann. ${ }^{272}$

Da im trockenen Putz die Carbonatisierung des Kalkes bereits abgeschlossen ist, können die Pigmente nicht einfach mit Wasser vermischt aufgetragen werden. Gerade im Außenbereich hätte dies zu einer nur sehr kurzen Haltbarkeit geführt - wohingegen die Dipinti mitunter einige Jahrzehnte überdauerten und auch heute noch fest mit dem Putz verbunden sind (Abb. F20). Alternativ dazu konnten sie als Temperafarben auf den trockenen Putz aufgebracht werden, also mit einem organischen Binder wie Leim oder Casein angerührt werden, wodurch ebenfalls eine gute Haltbarkeit erzielt wurde. Gerade auf den im Außenbereich häufig anzutreffenden Putzen mit hydraulischen Zuschlägen war die Anwendung organischer Bindemittel zwingend nötig. ${ }^{273}$ Eine weitere Alternative, die dem Fresko am nächsten kommt, besteht darin, die Pigmente mit Kalkwasser anzurühren, sodass diese zwar nicht mit dem Kalk des Putzes abbinden, aber dennoch durch die Bildung von Calciumcarbonat fixiert werden. ${ }^{274}$ Schließlich brachte auch die häufig beobachtete Vorbereitung der Beschreibfläche mit einem Auftrag aus Kalkmilch den technischen Vorteil mit sich, eine Art sekundäre Frescomalerei zu ermöglichen, da - ähnlich wie durch Kalkwasser - die Pigmente durch die Carbonatisierung gebunden werden. ${ }^{275}$

Der Überblick über die verschiedenen verwendeten und möglichen Materialien zeigt, dass die Anbringung von Dipinti ein hohes Maß an Sachverstand erforderte. Die Pigmente, die für die verwendeten Farben benötigt wurden, gehörten zu den billigsten oder konnten sogar kurzfristig eigens hergestellt werden. Die richtige Vorbereitung der Materialien und die Zubereitung einer Farbe, die mit dem Untergrund kompatibel ist, ist mit spezifischen Kenntnissen verknüpft. Das zeigt sich auch an misslungenen

270 Vitr. 7,10,2; Plin. nat. 35,43. Plinius erwähnt in 28,63, dass besonders guter Leim aus Stierohren und -genitalien hergestellt werden könne, was Klinkert in einem Experiment mit Stierohren nachvollzogen hat: Klinkert 1957, 139.

271 Vgl. Klinkert 1957, 121-122. 133; Ling/Ling 2000, 57-58.

272 Wülfert 2013.

273 Vgl. Klinkert 1957, 134.

274 Ling/Ling 2000, 51.

275 Dazu: Emmenegger 1998, 123. 
Dipinti, wie etwa CIL IV 123, das mit zu dünnflüssig angerührter Farbe aufgetragen wurde (Abb. F25).

\subsubsection{Schriftarten und Gestaltung}

Relevante Merkmale der Dipinti sind die verwendeten Schriftarten sowie deren Spielräume, das Layout, die verwendeten Farben, der Grad an Formalisierung durch Abkürzungen und Ligaturen und das Verhältnis der gesprochenen Sprache zur Schrift. An erster Stelle stehen die zahlreichen programmata, gefolgt von edicta munerum, Grüßen, Mietannoncen, öffentlich ausgestellten Listen, cacator-Inschriften sowie weiteren Inschriften unterschiedlichen Inhaltes. Weil die Befunde in Pompeji und Herculaneum singulär sind, ist es oft nicht möglich zu entscheiden, ob es sich bei verschiedenen Ausprägungen der Buchstabenformen gemalter Inschriften um diachronische, diastratische oder diaphasische Varietäten handelt. ${ }^{276}$

\subsubsection{1 programmata}

Sowohl unter den programmata recentiora als auch unter den edicta munerum lassen sich einem Großteil der Dipinti bestimmte Gestaltungsmerkmale zuordnen. Fast alle können der scriptura actuaria zugeordnet werden. Dennoch zeigt sich, dass auch in diesem Rahmen ein breiter Spielraum besteht. So finden sich auf der einen Seite Buchstaben, deren Füße und Serifen in fließenden Übergängen und mit weicher Kehlung angeschlossen sind und die so einen sehr eleganten Eindruck hervorrufen. ${ }^{277}$ Dies fällt unter anderen bei einem programma für Lollius (CIL IV 7868) an der Fassade von IX 11, 2 (Abb.5) auf. Bei anderen ist deutlich zu erkennen, dass diese nachträglich an die senkrechten Hasten angesetzt wurden, wie dies in extremer Weise bei einem programma für Popidius Secundus (CIL IV 7489) neben Eingang II 1, 1 ins Auge springt (Abb. 6). Bei diesem Dipinto scheinen die Buchstaben mit mehreren Zügen und in großer Geschwindigkeit ausgeführt worden zu sein, wobei deutlich die Abdrücke der einzelnen Pinselborsten zu erkennen sind. Es kommen jedoch nicht nur die Extreme, sondern auch alle Zwischenstufen vor. Ein weiteres signifikantes Merkmal besteht in der unterschiedlichen Behandlung von Haar- und Schattenstrichen. Bei CIL IV 7504 (Abb.7) sind die Linien vergleichsweise schmal und fast ohne Unterscheidung der Linienstärken gezogen. Bei CIL IV 7804 (Abb. 8) dagegen wird durch unterschiedliche Strichdicken ein dynamisches Schriftbild erzeugt und durch die Verlängerung der Haarstriche von $A$ und $M$ nach unten noch betont.

276 In Anlehnung an den linguistischen Begriff der Varietäten nach Eugenio Coseriu: Coseriu 1980, 111-112. Der Vergleich sollte allerdings nicht zu einer Parallelbetrachtung von Sprache und Schrift führen, sondern lediglich darauf aufmerksam machen, dass auch in der Schriftgestaltung verschiedene Dimensionen in Betracht zu ziehen sind.

277 Zur Beziehung zwischen Serifen und dem Schreiben mit Pinseln: Catich 1968. 
Auch mit der Breite der Buchstaben und der Laufweite der Schrift konnten unterschiedliche Effekte erzielt werden: Während die Buchstaben in CIL IV 7851 (Abb.9) breit angelegt sind und trotz Zerstörung und ungünstigem Maßstab der Abbildung noch zu erkennen sind und sich in CIL IV 7387 (Abb.10) auch durch kleine eingefügte Verzierungen auszeichnen, ergibt sich in einigen Fällen ein ganz anderes Bild. In CIL IV 7579 (Abb. 11) östlich von Eingang II 4, 7, an der N-Seite der praedia Iuliae Felicis, sind die Buchstaben im Verhältnis zur Breite so hoch und noch dazu so eng aneinandergerückt, dass die senkrechten Hasten sehr stark betont werden. Für einen Passanten, der sich der Fassade entlang der Via dell'Abbondanza näherte, sind dadurch die Buchstaben deutlich schwieriger zu differenzieren. Doch auch hier zeichnen sich die

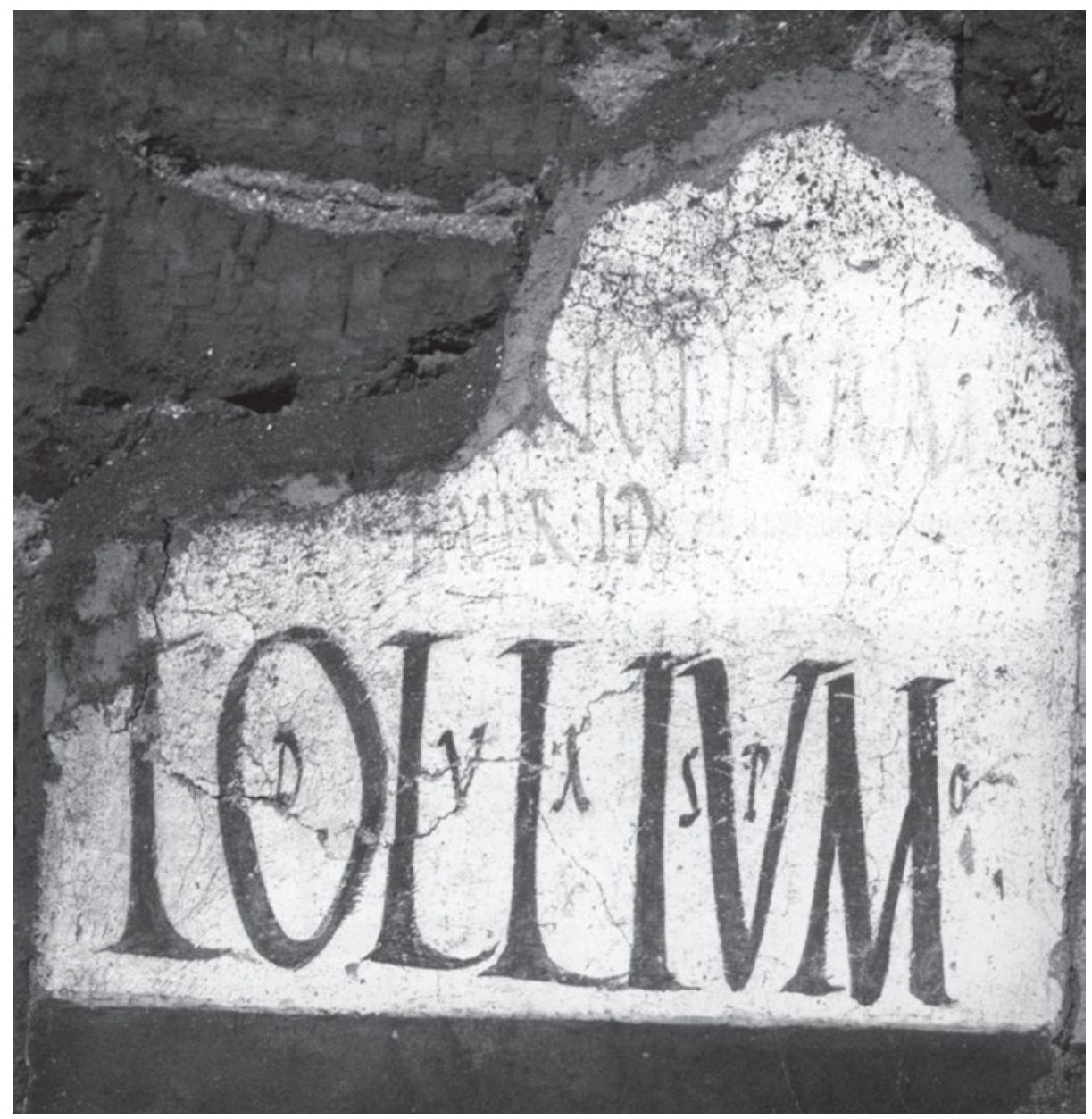

Abb. 5: CIL IV 7868 an der Fassade von IX 11, 2. @ Su concessione del Ministero per i Beni e le Attività Culturali e per il Turismo - Parco archeologico di Pompei. Jegliche weitere Reproduktion oder Duplikation ist untersagt. 




Abb. 6: CIL IV 7487-7490 neben Eingang II 1, 1. ( Su concessione del Ministero per i Beni e le Attività Culturali e per il Turismo - Parco archeologico di Pompei. Jegliche weitere Reproduktion oder Duplikation ist untersagt.

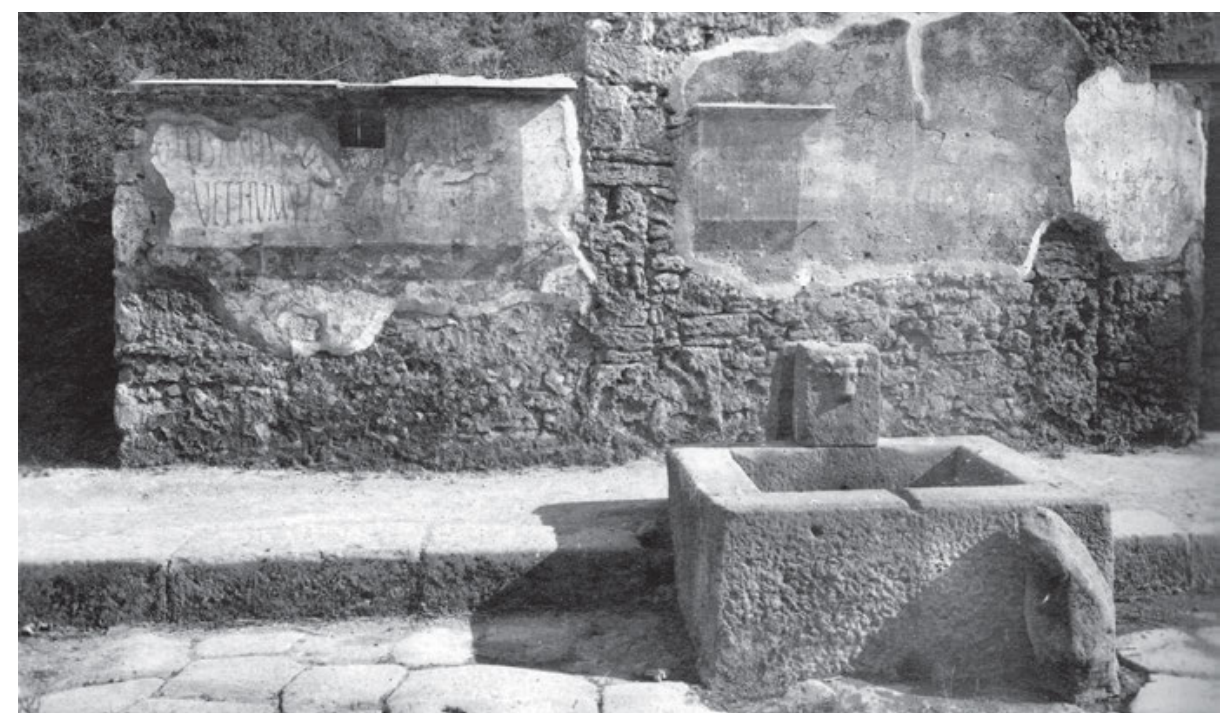

Abb.7: II 1, 2-3 mit CIL IV 7499, 7502 und 7504. (c) Su concessione del Ministero per i Beni e le Attività Culturali e per il Turismo - Parco archeologico di Pompei. Jegliche weitere Reproduktion oder Duplikation ist untersagt. 


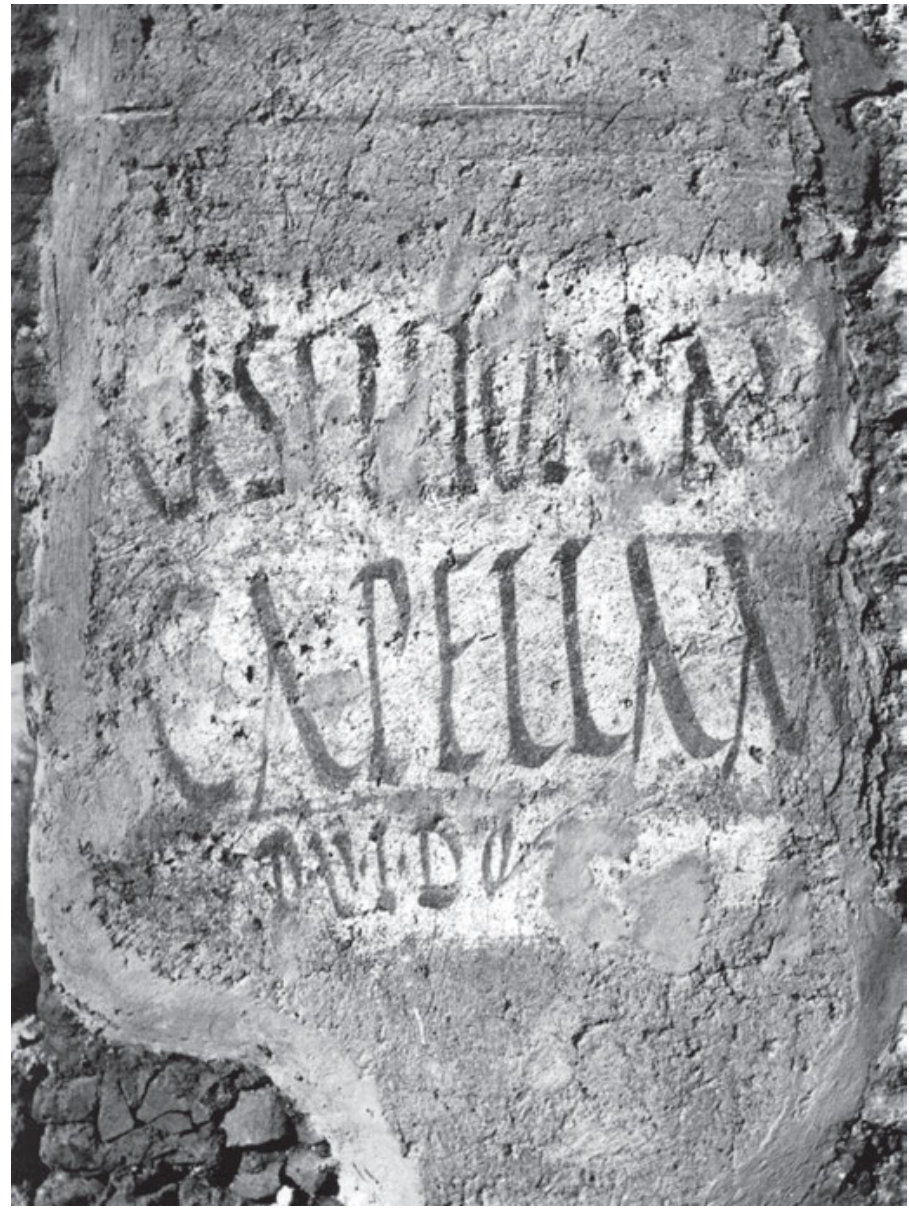

Abb. 8: CIL IV 7803 und 7804 bei III 7,5 . (c) Su concessione del Ministero per i Beni e le Attività Culturali e per il Turismo Parco archeologico di Pompei. Jegliche weitere Reproduktion oder Duplikation ist untersagt.

Buchstaben durch eine elegante Anlage aus und die Schreiber spielten sogar mit den Zwischenräumen, indem sie das letzte V verkleinert zwischen $\mathrm{N}$ und M schrieben. ${ }^{278}$ Die Dimensionen der programmata variieren stark: Die Buchstaben der kleinsten sind unter $10 \mathrm{~cm}$ hoch, während andere bis zu einen halben Meter messen. ${ }^{279}$ Die meisten Exemplare zeichnen sich durch ein sorgfältiges Arrangement aus, was darauf schließen lässt, dass dieses vor der Anbringung zumindest in groben Zügen festgelegt wurde. ${ }^{280}$ Auch in allen Fällen, in denen der Untergrund im Voraus weiß getüncht

278 Dass es sich nicht um ein Versehen, sondern um ein bewusst eingesetztes Gestaltungsmittel handelte, wird daran deutlich, dass der Abstand zwischen $\mathrm{N}$ und $\mathrm{M}$ größer ist, als zwischen den übrigen Buchstaben.

279 Mouritsen 1988, 31; für CIL IV 7553 wird eine Höhe von $50 \mathrm{~cm}$ angegeben, zu den größten programmata gehören auch CIL IV 7588 und 7589, beide an der N-Seite des campus II 7. Laut CIL sind die Buchstaben der Namen 43 bzw. $44 \mathrm{~cm}$ hoch.

280 Wallace 2005, IX; Mouritsen 1988, 31. 




Abb. 9: CIL IV 7851 bei IX 7, 9. (C) Su concessione del Ministero per i Beni e le Attività Culturali e per il Turismo - Parco archeologico di Pompei. Jegliche weitere Reproduktion oder Duplikation ist untersagt.

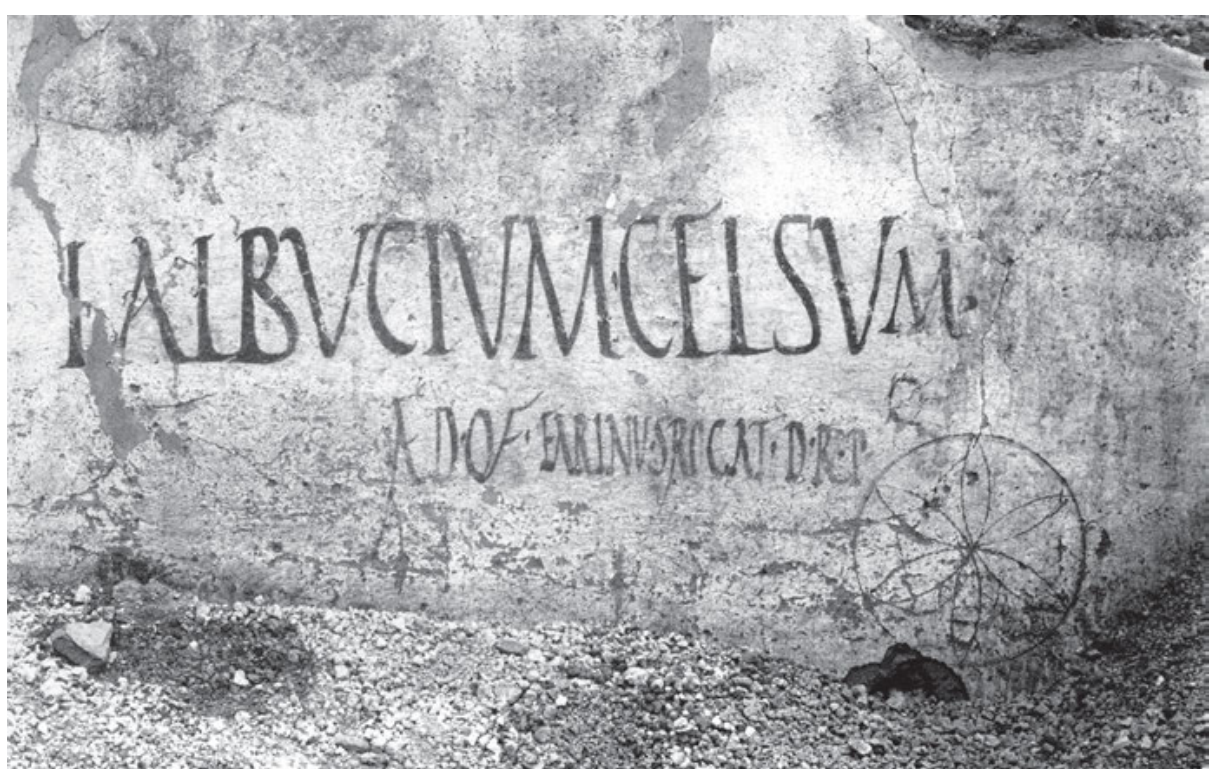

Abb.10: CIL IV 7837 bei I 19, 4. (C Su concessione del Ministero per i Beni e le Attività Culturali e per il Turismo - Parco archeologico di Pompei. Jegliche weitere Reproduktion oder Duplikation ist untersagt. 


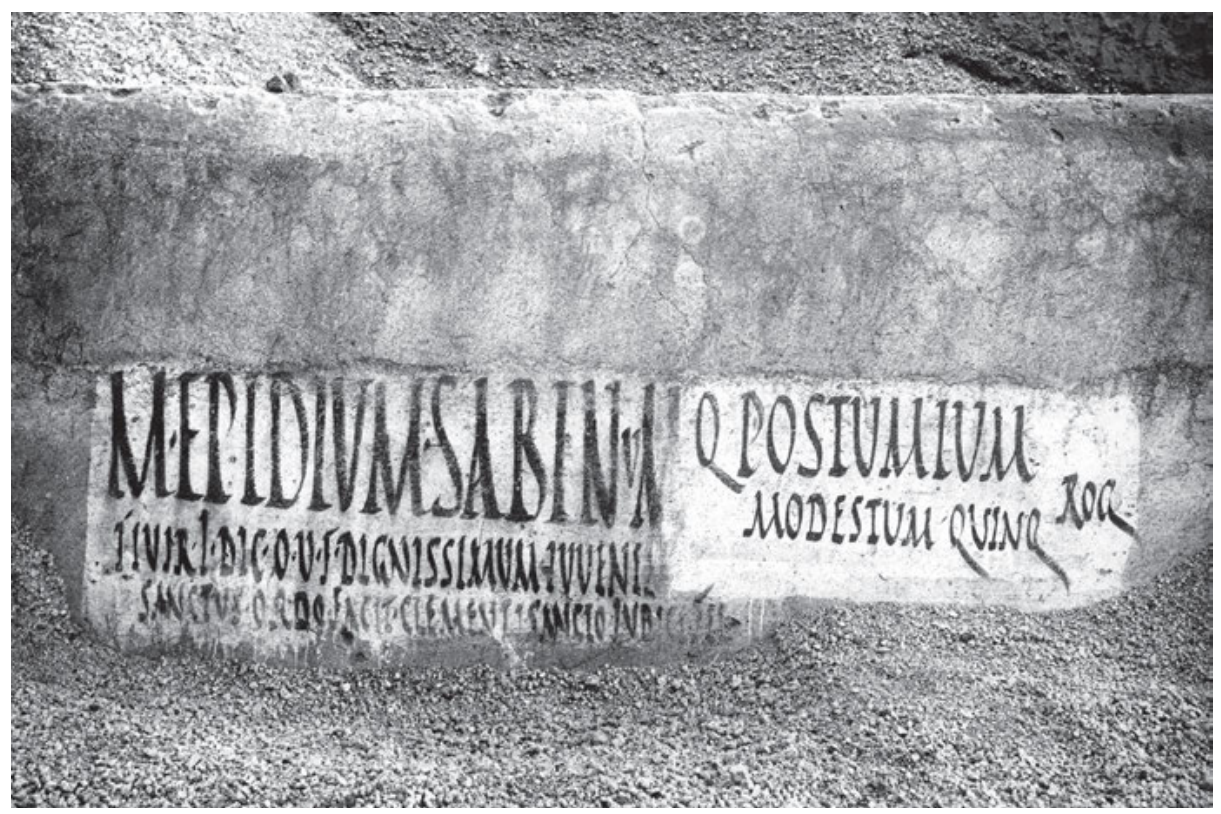

Abb. 11: CIL IV 7579 und 7580 bei II 4, 7. (C) Su concessione del Ministero per i Beni e le Attività Culturali e per il Turismo - Parco archeologico di Pompei. Jegliche weitere Reproduktion oder Duplikation ist untersagt.

wurde, muss vorher überschlagen worden sein, wie viel Platz benötigt werden würde, auch wenn man nachher davon abwich. ${ }^{281}$

Die weiß getünchten Flächen bilden in vielen Fällen nicht nur die chemische Grundlage für die Anbringung, sondern waren Teil der Gestaltung: So wurde etwa die Fläche für CIL IV 7580 (Abb.11) sorgfältig und mit geraden Rändern gezogen. In einigen Fällen wurde sie auch als tabula ansata gestaltet, zum Beispiel bei CIL IV 7627, 7628 und 7630 an der Casa di Trebius Valens (III 2, 1) (Abb. 38). Die tabulae dienen als optische Rahmung und heben die Inschrift von der Wand ab. ${ }^{282}$ Dies gilt in geringerem Maße auch bei einfachen Rechtecken, die nicht durch die angesetzten Henkel als tabulae ansatae gestaltet wurden, aber dennoch auch einen Rahmen für den Dipinto schufen.

In allen Fällen ist die ordinatio eng mit der sprachlichen Form und dem Inhalt verknüpft. Der Inhalt war hochgradig formalisiert und bestand aus mehreren Teilen, von denen fast alle - bis auf den Namen des Kandidaten - wegfallen konnten. ${ }^{283}$ Der Name nahm fast immer eine besonders prominente Stelle ein, indem er zuerst genannt und

281 Staccioli 1992, 43.

282 Vgl. dazu: Schepp 2009, 114. 116. Weitere und besonders sorgfältig gestaltete tabulae ansatae finden sich bei I 7, 19 (CIL IV 7256) und I 7, 13 (CIL IV 7241).

283 Mouritsen 1988, 31. 




Abb.12: CIL IV 9885 bei II 4, 7. (C) Su concessione del Ministero per i Beni e le Attività Culturali e per il Turismo - Parco archeologico di Pompei. Jegliche weitere Reproduktion oder Duplikation ist untersagt.

meist deutlich größer als der Rest des Textes geschrieben wurde. ${ }^{284}$ Ansonsten konnten fast alle Bestandteile, also das angestrebte Amt, die üblichen Qualifikationen dignum rei publicae und virum bonum, die Bitte oro vos faciatis und die Namen der rogatores samt Zusatz roga(n)t abgekürzt werden, was in der Regel auch geschah. Insbesondere oro vos faciatis und aedilis wurden in konventionellen nexus wiedergegeben, die zum Beispiel in CIL IV 9885 (Abb. 12) und CIL IV 9883 (Abb. 13) gut zu erkennen sind. ${ }^{285}$ Durch dieses Bausteinsystem ergibt sich eine zwar stark formularisierte Anlage, die jedoch, was die Anordnung der einzelnen Bestandteile betrifft, viel Spielraum lässt. Es wurde oft damit gespielt, den Namen in eine Zeile zu schreiben und den übrigen Inhalt in mehreren Zeilen, die zusammen ebenso hoch sind, daneben zu platzieren, wie zum Beispiel in CIL IV 9884 (Abb.14). Auffällig ist in dieser Hinsicht auch CIL IV 7868 (Abb. 5), bei dem kleinere Buchstaben in und zwischen die größeren des Namens geschrieben wurden.

Es finden sich zahlreiche Beispiele, in denen die Schreiber der programmata sich eng an der bereits bestehenden Wandgestaltung orientierten. CIL IV 9883 und 9884 (Abb. 13. 14) an der Fassade von II 1, 10 sind beide bis auf wenige $\mathrm{cm}$ an die Kante zwischen der oberen und der unteren Zone der Wandfläche herangerückt und nehmen

284 Vgl. Staccioli 1992, 48; Wallace 2005, XI.

285 Mouritsen 1988, 31; Staccioli 1992, 45. 


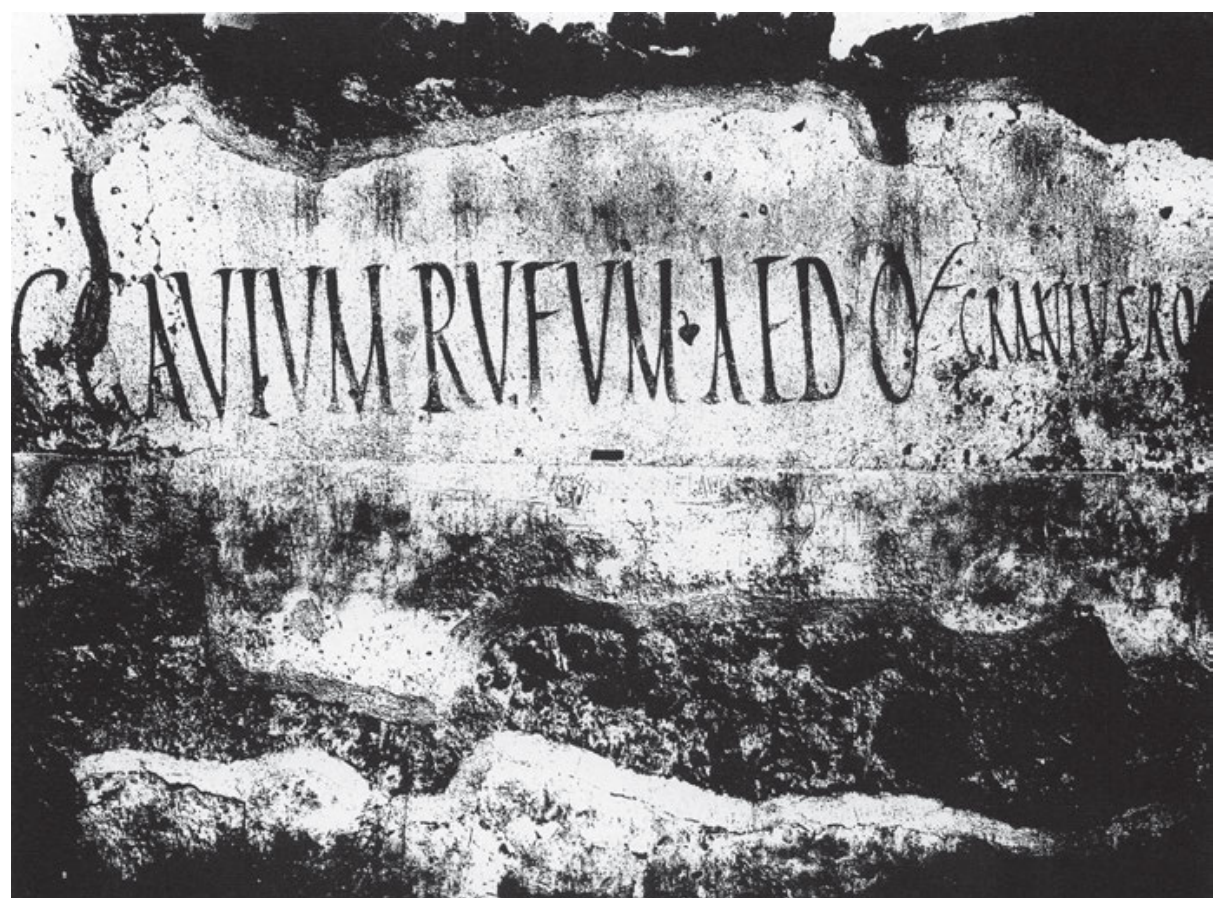

Abb. 13: CIL IV 9883 bei Eingang II 1, 10. @ Su concessione del Ministero per i Beni e le Attività Culturali e per il Turismo - Parco archeologico di Pompei. Jegliche weitere Reproduktion oder Duplikation ist untersagt.

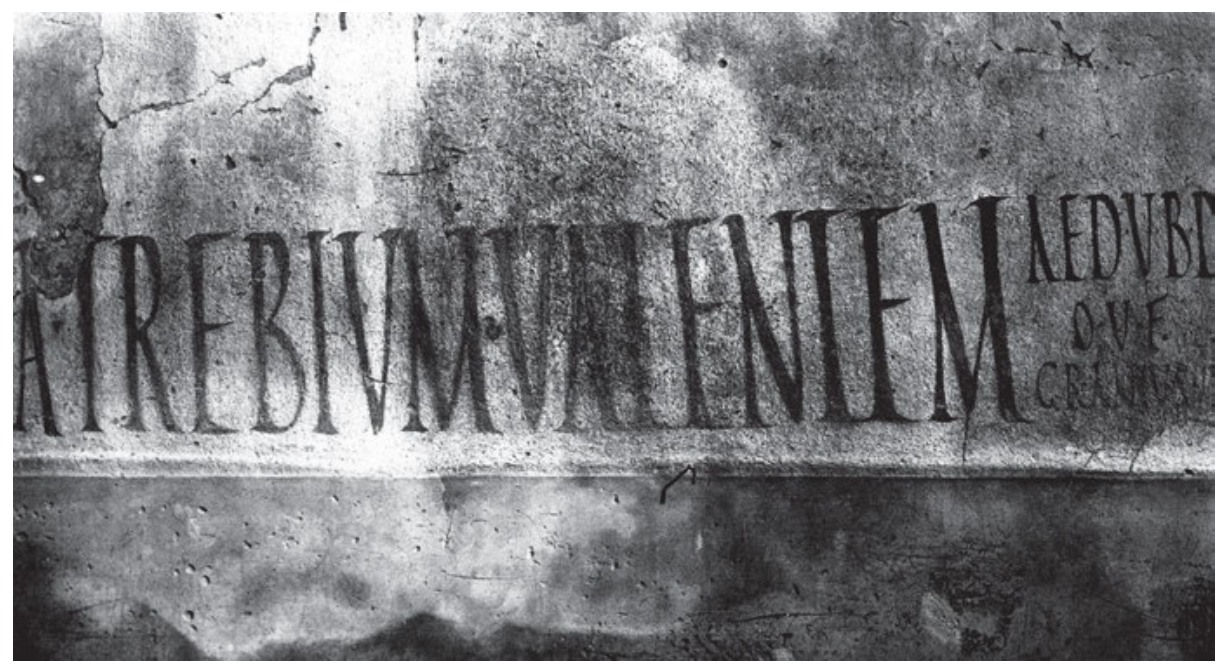

Abb. 14: CIL IV 9884 bei Eingang II 1, 10. @ Su concessione del Ministero per i Beni e le Attività Culturali e per il Turismo - Parco archeologico di Pompei. Jegliche weitere Reproduktion oder Duplikation ist untersagt. 


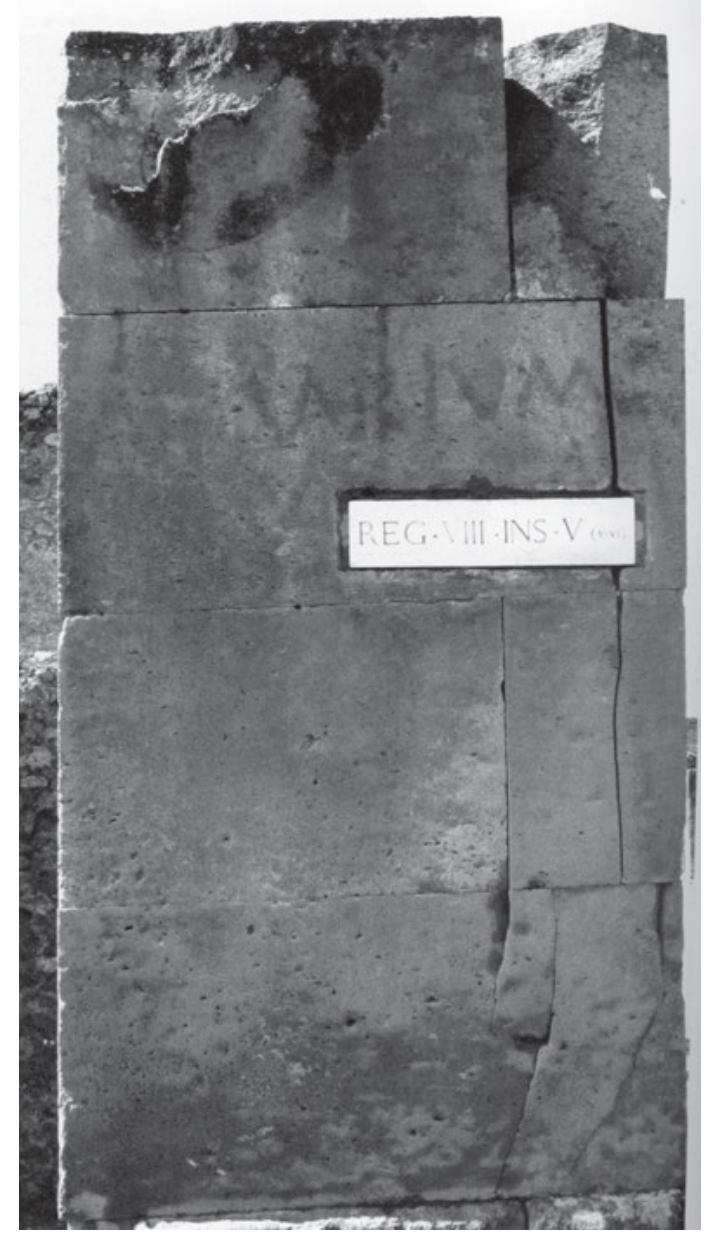

Abb. 15: CIL IV 61, programma antiquissimum an der Nordostecke von insula VIII 5, zur Via dei Teatri hin. (C) Su concessione del Ministero per i Beni e le Attività Culturali e per il Turismo - Parco archeologico di Pompei. Jegliche weitere Reproduktion oder Duplikation ist untersagt.

so Bezug darauf. ${ }^{286}$ Wenn die untere Zone in Orthostaten gegliedert war, nahmen die Maler auch hierauf immer wieder Bezug, so etwa an der Casa di Trebius Valens (III 2, 1) (Abb. 38) und an der Fullonica di Stephanus (I 6, 7) (Abb. 31): Dort ist ein programma (CIL IV 7172) genau in den oberen Bereich eines Feldes eingepasst, während ein weiteres (CIL IV 7173) zwei dieser Breiten einnimmt. Einen seltenen Fall stellt die Fassade der Casa dei Cei (I 6, 15) dar (Abb. F26), die eine mit Stuck plastisch ausgeführte Wandgliederung aufweist. In mehrere der stuckierten Quaderblöcke bzw. Rechteckfelder sind programmata eingeschrieben: CIL IV 7191.7187 und 7290.

Innerhalb Pompejis bilden die ca. 100 sogenannten programmata antiquissima, die aller Wahrscheinlichkeit nach aus den Jahrzehnten unmittelbar nach der Gründung

286 Diese beiden programmata müssen von demselben Schreiber gemalt worden sein, was nicht nur an den hederae, sondern auch besonders an den Formen von $<\mathrm{M}>$ und $<\mathrm{R}>$ gut zu erkennen ist. 


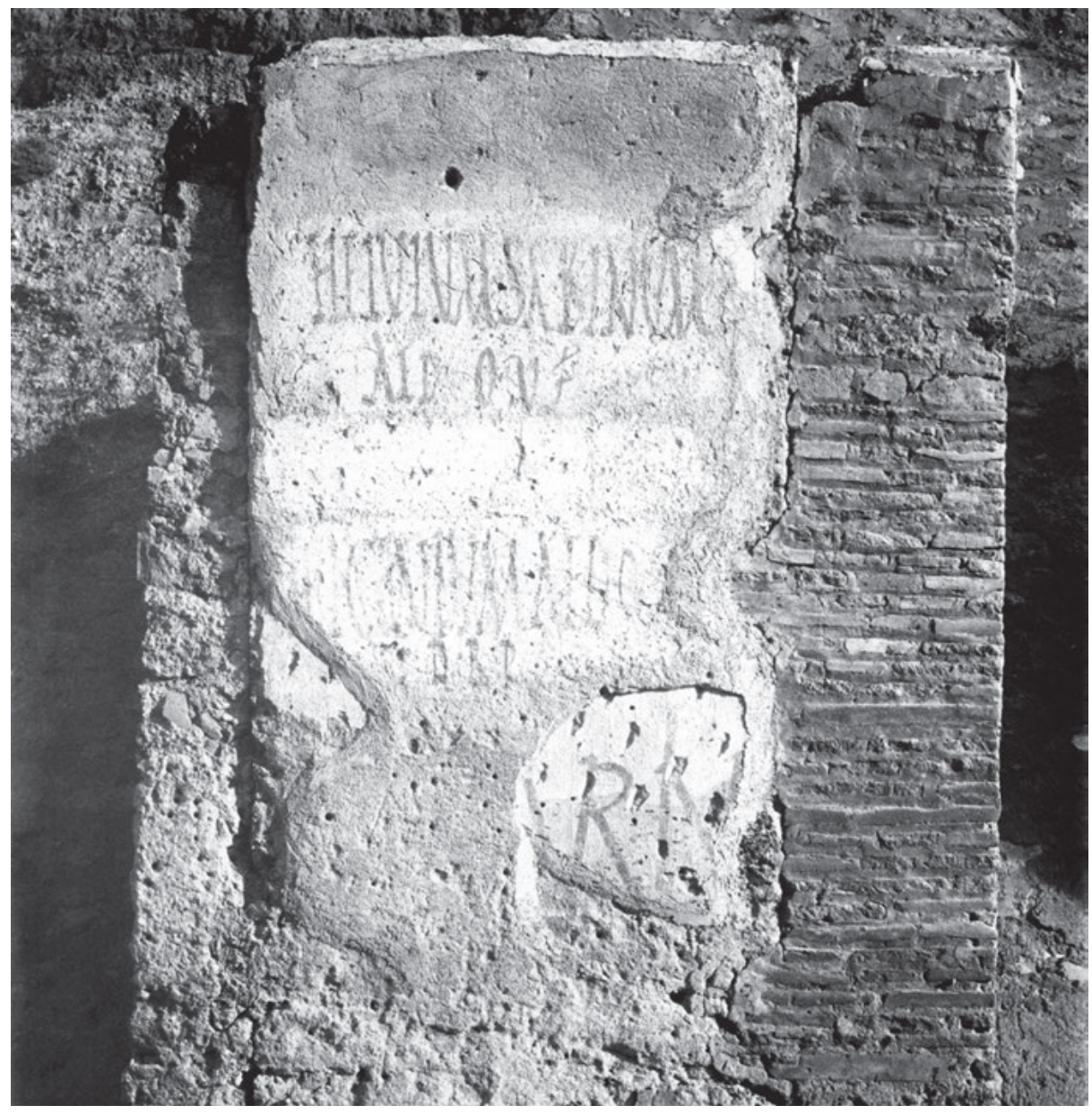

Abb. 16: CIL IV 7121, programma antiquissimum auf einer älteren Putzschicht neben Eingang III 1, 1, von CIL IV 7593 und 7594 überlagert. @ Su concessione del Ministero per i Beni e le Attività Culturali e per il Turismo - Parco archeologico di Pompei. Jegliche weitere Reproduktion oder Duplikation ist untersagt.

der Kolonie stammen, eine eigene Gruppe. ${ }^{287}$ Neben inhaltlichen Unterschieden sowie bezeichnenden republikanischen Schreibweisen weisen sie auch eigentümliche gestalterische Merkmale auf, die sie deutlich von den späteren programmata recentiora absetzen. Zum einen finden sich nur diese und keine programmata recentiora auf den nackten Tuff gemalt (Abb. 15). ${ }^{288}$ Besonders auffällig ist jedoch das von der actuaria völlig verschiedene Schriftbild, das Zangemeister als „maximas, crassas, quales

287 Im CIL IV sind sie unter den Nummern 1-83, 3341-3354, 6601-6604, 7114-7127 und 9822-9827 aufgeführt. Zur Datierung: Mouritsen 1988, 81.

288 Aber auch auf Putz: tectorio opere (Zangemeister CIL IV Hauptband, S.1). 
Oscae illae sunt, et eius formae, quam exeunte republica Romana in usu fuisse inscriptiones et pictae et lapidariae docent" beschreibt. ${ }^{289}$ Diese Gestaltungsweise, die noch einer völlig anderen Praxis der Schriftgestaltung verpflichtet war, wird auch an den wenigen Beispielen, die sich erhalten haben, deutlich. Während viele der programmata antiquissima auf Putzschichten aufgemalt sind, die später überputzt wurden (Abb. 16) und daher kurz vor 79 n. Chr. wohl nicht mehr zu sehen waren, standen doch die alten Dipinti, die direkt auf die Tuffblöcke gemalt waren, wie etwa CIL IV 30 (Abb. F22), den Pompejanern weiterhin vor Augen.

\subsubsection{2 edicta munerum}

Auch bei den edicta munerum variierten die Schreiber die Schriftarten. Allerdings ist es bei diesen noch schwieriger, zuverlässige Aussagen über die Gestaltung zu treffen, da von den 83 Zeugnissen nur 23 fotografisch dokumentiert sind. Im CIL wird bei einigen Beispielen die ordinatio wiedergegeben, allerdings nicht ausreichend genau. Oft werden auch die Buchstabenhöhen der verschiedenen Zeilen angegeben. Zudem findet sich im einleitenden Abschnitt in CIL IV S. 70 die Bemerkung „pictae sunt quod sciam omnes in tectorio eodem colore eademque litterarum specie atque candidatorum programmata recentiora“. Um die Gestaltungsmerkmale der edicta munerum zu verdeutlichen, seien drei Exemplare vorgestellt. Ein gut dokumentiertes Beispiel stellt CIL IV 7992 an der Fassade der Casa di Trebius Valens (III 2, 1) dar (Abb.43), das sich fast vollständig erhalten hatte und anhand des CIL-Eintrages, der Rekonstruktion bei Spinazzola und von Fotografien beschrieben werden kann. ${ }^{290}$ Es ist insgesamt fast $90 \mathrm{~cm}$ hoch und 3,12 m breit. Die Buchstaben der ersten Zeile, die den Namen des Spielgebers enthielt, sind laut CIL $48 \mathrm{~cm}$ hoch, während die der zweiten Zeile $11 \mathrm{~cm}$ und die der dritten Zeile $10 \mathrm{~cm}$ messen. Die erste Zeile war rot geschrieben, in der zweiten Zeile wurde bis einschließlich et und in der dritten für die letzten vier Wörter schwarze Farbe verwendet, sodass der Name des zweiten Spielgebers sowie Ort und Datum der Spiele optisch von den Titeln des ersten Spielgebers und den Zusatzinformationen zum Programm abgesetzt waren. Genannt werden außer den Namen der beiden Veranstalter, der Zeit und dem Ort auch die Anzahl der kämpfenden Gladiatorenpaare - wie üblich abgekürzt mit glad(iatorum) par(ia) - eine venatio und vela, die anscheinend eine eigene Erwähnung wert waren. ${ }^{291}$ Die unteren beiden Zeilen schließen bündig mit der ersten Zeile ab, die dritte Zeile ist jedoch links ca. $40 \mathrm{~cm}$ eingerückt. Die Schriftart der ersten Zeile kann noch als actuaria bezeichnet werden, weist

$289 \mathrm{Vgl}$. Zangemeister CIL IV, S.1. Zu den Merkmalen der programmata antiquissima: Mouritsen $1988,80$.

290 Für eine Beschreibung des Kontextes siehe Kapitel 4.1.1. Ein großer Teil dieser Fassade und der darauf angebrachten Dipinti wurde 1943 zerstört. Spinazzola 1953, Taf. 9; Varone/Stefani 2009, 238.

291 Dass das Aufspannen von vela nicht selbstverständlich war, obwohl entsprechende Vorrichtungen dauerhaft installiert waren, bemerkt auch Rainer Graefe, der feststellt, dass nur in 20 edicta vela ankündigt werden: Graefe 1979, 8. 68-70. 
jedoch hinsichtlich der Seitenverhältnisse der einzelnen Buchstaben, der Behandlung der einzelnen Züge und der Winkel Ähnlichkeiten mit der quadrata oder monumentalis auf. Die zweite und dritte Zeile sind dagegen den in unmittelbarer Umgebung befindlichen programmata viel ähnlicher. Die Schreiber nutzen hier also verschiedene Parameter, um die Inschrift effektvoll zu gestalten: Anbringungshöhe, Größe, farbliche Kontrastierung und unterschiedliche Buchstabenformen.

Knapp $90 \mathrm{~cm}$ rechts von CIL IV 7992 befindet sich ein weiteres edictum (CIL IV 7993). ${ }^{292}$ Beide Dipinti weisen einige Gemeinsamkeiten auf. Die Dimensionen sind vergleichbar: CIL IV 7993 ist $2 \mathrm{~m}$ breit, die oberste Zeile $49 \mathrm{~cm}$ hoch und die beiden folgenden 10 und $7 \mathrm{~cm}$. Auch die Anordnung in drei Zeilen entspricht dem, was bei CIL IV 7992 beobachtet werden konnte. ${ }^{293}$ Die Buchstabenformen weisen ebenfalls Gemeinsamkeiten auf, wobei jedoch die der ersten Zeile etwas schmaler und in ihren Formen stärker der actuaria verpflichtet sind als in 7992. Der Schreiber verwendete ebenfalls sowohl rote als auch schwarze Farbe, und auch hier lässt sich beobachten, dass dadurch der Name des Stifters hervorgehoben werden sollte, da in Zeile 2 alles außer diesem in Schwarz geschrieben war. Ein Unterschied besteht allerdings darin, dass in der ersten Zeile nicht der Name, sondern ein Hinweis auf den Anlass prominent erscheint.

Ähnliche Gestaltungsmerkmale, aber wiederum eine andere Textverteilung weist CIL IV 9983a auf, das auf die Fassade der Gräber OS 9 und 11 geschrieben wurde (Abb. F27). Auch hier wird in der ersten Zeile die Anzahl der Gladiatorenpaare angegeben, die bei den Spielen auftraten. ${ }^{294}$ Anders als vielen anderen edicta munerum wird hier jedoch noch vor den Gladiatoren der Veranstaltungsort genannt. Es handelte sich nämlich nicht um Pompeji, sondern um Cumae. Folglich war gerade diese Information auch für die intendierten Leser besonders wichtig und gehörte an die erste Stelle. ${ }^{295}$ Diese Art, Informationen von unterschiedlicher Priorität gezielt an den möglichen Stellen der Ankündigungen zu platzieren, ist charakteristisch für die Spielankündigungen. ${ }^{296}$ Da der Veranstaltungsort außerhalb Pompejis lag, wurde die Ankündigung nicht nur außerhalb der Stadt angebracht, sondern auch eine andere Reihung der Informationen gewählt.

292 Wie lange diese beiden Dipinti tatsächlich nebeneinander zu sehen waren, ist nicht endgültig zu klären. Das edictum des Gnaeus Alleius Nigidius Maius (CIL IV 7993) stammt vermutlich aus der Zeit zwischen 62 und 69 n. Chr. (Franklin 1997, 444 und Sabbatini Tumolesi 1980, 38-40). Für die Ankündigung der Spiele der Lucreti (CIL IV 7992) ist ein Zeitraum kurz vor dem Tod Neros wahrscheinlich (Mouritsen 1988, 35); für eine frühere Datierung: Sabbatini Tumolesi 1980, 29-31.

293 Größenangaben und Hinweise auf die Anordnung im CIL können bestätigen, dass diese Art der Gliederung üblich war. Vgl. zum Beispiel die Einträge zu CIL IV 1180, 1190, 3881, 3883, 7990 oder 7991. 294 Die Abbildungen in Varone/Stefani 2009, 494-500 zeigen nicht alle Details, sodass hier die Angaben im CIL besonders wichtig sind. Dort entsteht allerdings der Eindruck, dass die beiden X in Zeile 1 durch den gemalten Gruß auseinandergerückt seien, was ein Blick auf die Fotos widerlegt.

295 Darauf weist auch Sabbatini Tumolesi hin: Sabbatini Tumolesi 1980, 117.

296 Vgl. dazu die Sammlung der Texte bei Sabbatini Tumolesi 1980. 


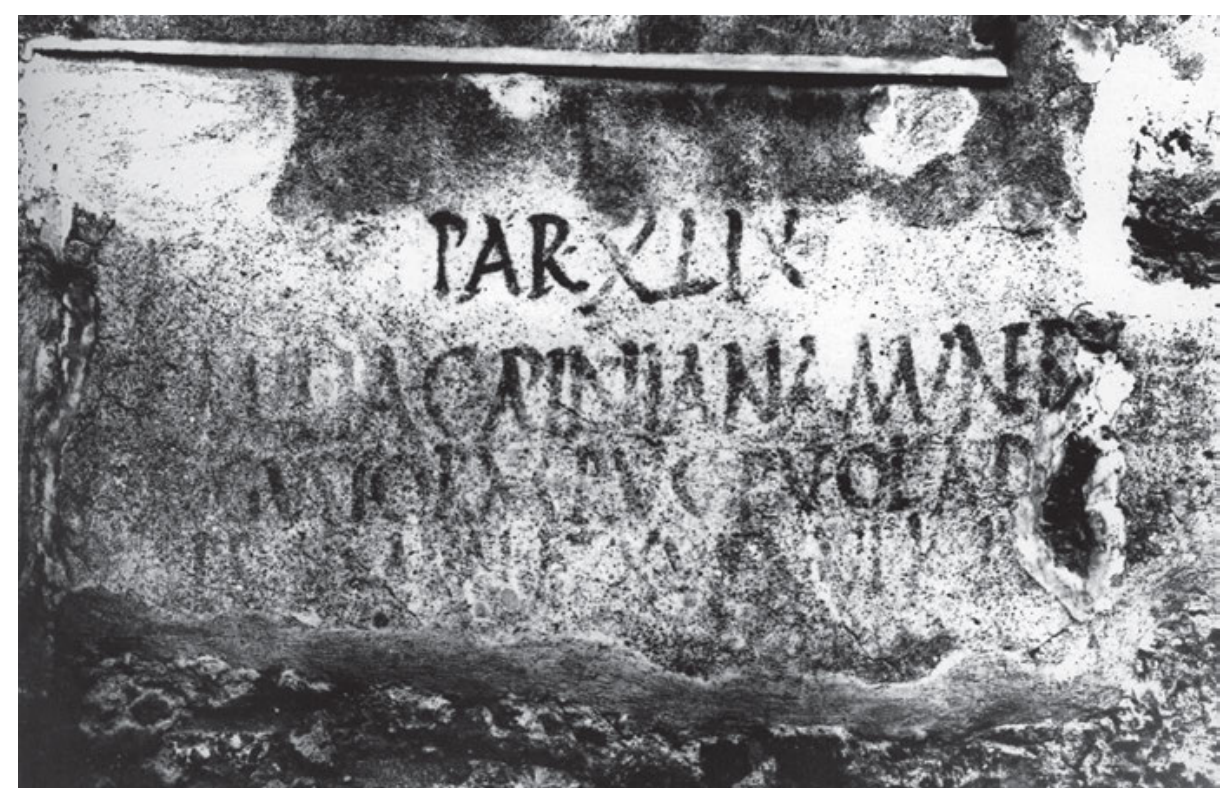

Abb. 17: CIL IV 7994, westlich von Eingang III 4, 2. (C) Su concessione del Ministero per i Beni e le Attività Culturali e per il Turismo - Parco archeologico di Pompei. Jegliche weitere Reproduktion oder Duplikation ist untersagt.

Der Wiedererkennungswert als edictum muneris wurden somit auch über die Form und Gestaltung generiert. Dies zeigt, dass das Schema durchaus variabel war und dass in einigen Fällen dem optischen Erscheinungsbild eine mindestens so große und deutliche Bedeutung beigemessen wurde wie dem eigentlichen Inhalt: Wenn es als gelungen empfunden wird, eine gleichbleibende Form mit immer ähnlichen Inhalten zu füllen, dann gewinnt die Form an sich ebenfalls inhaltliche Qualitäten. Das wird besonders an dem zweiten Beispiel deutlich. Der Begriff dedicatione gibt alleine keinerlei Hinweisdarauf, was hier angekündigt wurde. Aufrgund der formalen Gestaltung war aber sofort klar, dass es sich um eine Spielankündigung handelte.

Auch unter den edicta munerum können mehrere Exemplare identifiziert werden, die nicht aus den letzten Jahren der Stadt stammen können. ${ }^{297}$ Diese können anhand von Bezügen auf das Kaiserhaus, prosopographischen Überlegungen, der Orthographie und der Paläographie in die augusteische Zeit oder kurz danach datiert werden. ${ }^{298}$ Deutlich wird dies zum Beispiel an CIL IV 7994, das an der Casa del Moralista zwischen den Eingängen III 4, 1 und III 4, 2 angebracht war (Abb.17). Das Schriftbild

297 Von diesen wurden einige in der Nekropole an der Porta Nocera gefunden, was Mouritsen dadurch erklärt, dass in den Nekropolen die Putzflächen beständiger waren und selten erneuert wurden: Mouritsen 1988, 36.

298 Mouritsen 1988, 36. 189 Anm. 153. Mouritsen nennt hierunter die edicta CIL IV 1196. 3881. 3882. 7994. 9969. 9979.9980 und 9981a. 
der edicta an Grab EN 10 (Abb. 51) in der Nekropole an der Porta Nocera war außergewöhnlich und den programmata antiquissima ähnlich. Die beiden K in dem dort angebrachten edictum CIL IV 9972 sind insbesondere den K in den oskischen Inschriften Ve 27 (Abb. 2) und Ve 28 besonders ähnlich, deren Schenkel ebenfalls sehr kurz sind und nur etwa ein Drittel der Buchstabenhöhe einnehmen. ${ }^{299}$ Die Schrift weist grobe, undynamische und serifenlose Formen auf. ${ }^{300}$ Das Grab wird augusteisch datiert, was bedeutet, dass bereits kurz nach seiner Errichtung die ersten Dipinti daran angebracht worden sein müssen. ${ }^{301}$

\subsection{Weitere Dipinti}

Weitere Bekanntmachungen weisen ähnliche Gestaltungsmerkmale auf: Mietannoncen, Listen der magistri vici et compiti, zwei Dipinti in Herculaneum sowie eine größere Anzahl an Grüßen. Bei den 42 publizierten Grüßen ist neben der räumlichen und inhaltlichen Nähe zu den programmata auch festzustellen, dass die gleichen Farben und die gleiche Schriftart verwendet wurde, wie bei diesen. Dies ist zweifellos dadurch zu erklären, dass sie von denselben Schreibern angebracht wurden.

Formal stellen insbesondere die beiden Mietannoncen CIL IV 138 und 1136 interessante Vergleichsmöglichkeiten dar. ${ }^{302}$ Während CIL IV 138 infolge der Ausgrabung verlorenging und heute nur noch in einer Abbildung bei Mazois ${ }^{303}$ überliefert ist, wurde CIL IV 1136 bereits im 18. Jh. abgenommen und befindet sich heute im Nationalmuseum in Neapel (Abb. F28). ${ }^{304}$ Trotz der Beschädigung durch den Transfer ist $\mathrm{zu}$ erkennen, dass das Layout dieser Inschrift in hohem Maße formalisiert ist. Die Grundlinien und H-Linien sind vorgezeichnet und die Höhe der einzelnen Buchstaben somit festgelegt. Der Text ist so auf die fünf Zeilen verteilt, dass auch hier ein Name in der ersten und größten Zeile als Aufhänger dient und die Details in immer kleiner

299 Vgl. Varone/Stefani 2009, 257-259.

300 CIL IV 9972. 9974. 9976. Das CIL vermerkt zu 9972: litteris crassis.

301 D’Ambrosio/De Caro 1983, EN 10. In den genannten edicta werden Spiele in Nuceria angekündigt, sodass zu der zeitlichen Differenz auch noch die Frage hinzukommt, inwieweit sich die Gestaltungspraktiken in den jeweiligen Städten unterschieden und ob für die Dipinti in Pompeji Maler von dort oder aus Nuceria beauftragt wurden. Vgl. Kapitel 4.3.

302 Beide Mietanzeigen stellten Unikate dar, da sie jeweils an der insula angebracht waren, auf die sie sich bezogen. Zur Lokalisierung insbesondere von CIL IV 138: Pirson 1999, 15-17. Inhaltlich ähnlich sind die eng verknüpften Dipinti CIL IV 806 und 807, über deren Gestaltung jedoch nur begrenzte Details bekannt sind.

303 Mazois III 1824 Taf. 1; die Treue dieser Darstellung ist natürlich fraglich. In Mazois II 1824, 1 ist unter anderen auch CIL IV 1136 abgebildet, die auch fotografisch dokumentiert ist (Varone/Stefani 2009, 212-213). Hier sind die Buchstabenformen recht präzise wiedergegeben. Insgesamt zeigt Mazois allerdings nicht viele Dipinti.

304 Entdeckt und abgenommen am 8. Februar 1756: Fiorelli 1860, 38. Siehe auch: Donati 1998, 305306 im Katalogeintrag zu Kat. 111. Weitere Fotos: Varone/Stefani 2009, 212-213. 
werdenden Zeilen folgen. Die letzte Zeile ist wieder etwas größer. ${ }^{305}$ Nur einzelne kalligraphisch ausgearbeitete Buchstaben wie das B am Anfang von Zeile 3 ragen über die H-Linie hinaus. Die Schrift ist eine ausgesprochen sorgfältig ausgeführte, vergleichsweise schlanke actuaria. Anders als bei den programmata recentiora und den edicta munerum ist kein Qualitätsunterschied zwischen der ersten Zeile und den niedrigeren folgenden Zeilen, die die Details beinhalten, festzustellen. Zudem war der Text zentriert, wobei das essentielle Wort locantur allein in Zeile 2 steht. ${ }^{306}$ Die geritzten Linien zeigen, dass die Inschrift im Vorhinein mit größter Sorgfalt geplant wurde, indem man sowohl die Höhe der einzelnen Zeilen als auch deren nahezu gleichbleibende Abstände voneinander festlegte. ${ }^{307}$

Sowohl in Pompeji als auch in Herculaneum wurden einige Dipinti gefunden, die als alba gedeutet werden können, also Namenslisten oder offizielle Bekanntmachungen unter Berufung auf aktuelle Beamte. ${ }^{308} \mathrm{Zu}$ den Dipinti CIL IV 7807, 9934a, 10489 und den von Pagano 1992 vorgestellten Fragmenten liegen Abbildungen vor, die mit Ergänzungen zu den Maßen anhand des CIL nähere Aussagen zur Gestaltung erlauben. ${ }^{309}$ Formal zeichnen sie sich dadurch aus, dass sie großteils in Schwarz geschrieben und sehr sorgfältig und ebenmäßig ausgeführt sind. Die Grundlinien und teilweise auch die H-Linien sind vorgezeichnet. ${ }^{310}$ Bei CIL IV 7807 (Abb.18), einer Auflistung der magistri vici et compiti sind die Zeilen 4 und 11, die vermutlich die Zwischenüberschriften bildeten, in roter Farbe geschrieben und entsprechen somit den üblichen rubricae. ${ }^{311}$ Deutlich unterscheidet sich auch die Schrift dieser beiden Zeilen vom Rest der Inschrift, sodass in diesem Fall von zwei (oder mehr) Arbeitsschritten auszugehen ist. CIL IV 9934a ist ganz offensichtlich nur ein kleiner Teil eines ursprünglich sehr viel größeren Textes, war aber sicherlich ebenfalls Teil einer ähnlichen Liste. ${ }^{312}$ Der in Herculaneum gefundene Dipinto CIL IV 10489 (Abb.F29) ist unvollständig erhalten. Es handelt sich um eine öffentlich bekanntgegebene Regulierungsmaßnahme der

305 Die Höhen der einzelnen Zeilen betragen laut CIL: $1.20 \mathrm{~cm}$; 2. keine Angabe; $3.10 \mathrm{~cm} ; 4.7 \mathrm{~cm}$ und $5.9,8 \mathrm{~cm}$.

306 Das CIL allein bietet hierfür keine zuverlässigen Informationen. So z. B. bei CIL IV 7208, wo dignus est rechtsbündig gesetzt ist, während es auf dem Foto fast mittig steht (Varone/Stefani 2009, 76).

307 Es handelt sich nicht um „flüchtig gemalte Dipinti“, wie Pirson meint: Pirson 1999, 15. In umso größerem Kontrast dazu erscheint das direkt darunter gemalte programma für Aulus Suettius Verus, das unebenmäßig wirkt.

308 CIL IV 60. 7807, 9934a. 10489 (Herculaneum) sowie die in Pagano 1992, 189-191 präsentierten Fragmente aus Herculaneum, vgl. auch Guadagno 1977. Fioretti 2012, 415-416. Auch das verlorene CIL IV 10488, das 10489 überdeckte, ist zu dieser Gruppe zu zählen. Bei CIL IV 60 und 9934a ist mit Sicherheit davon auszugehen, dass sie zur Zeit des Vesuvausbruches nicht zu sehen waren, da sie überdeckt oder abgenommen worden waren.

309 Pagano 1992, 189-190 Abb.1-4; Varone/Stefani 2009, 382. 511. Taf. 48. CIL IV 60 scheint der Darstellung im CIL nach ähnlich wie CIL IV 7807 aufgebaut gewesen zu sein.

310 Besonders deutlich bei CIL IV 7807.

311 Schmidt 1894, Sp. 1332-1336.

312 Fioretti 2012, 415-416 Anm. 19. Vgl. dazu auch die kurze Bemerkung in Solin 1973b, 275. 


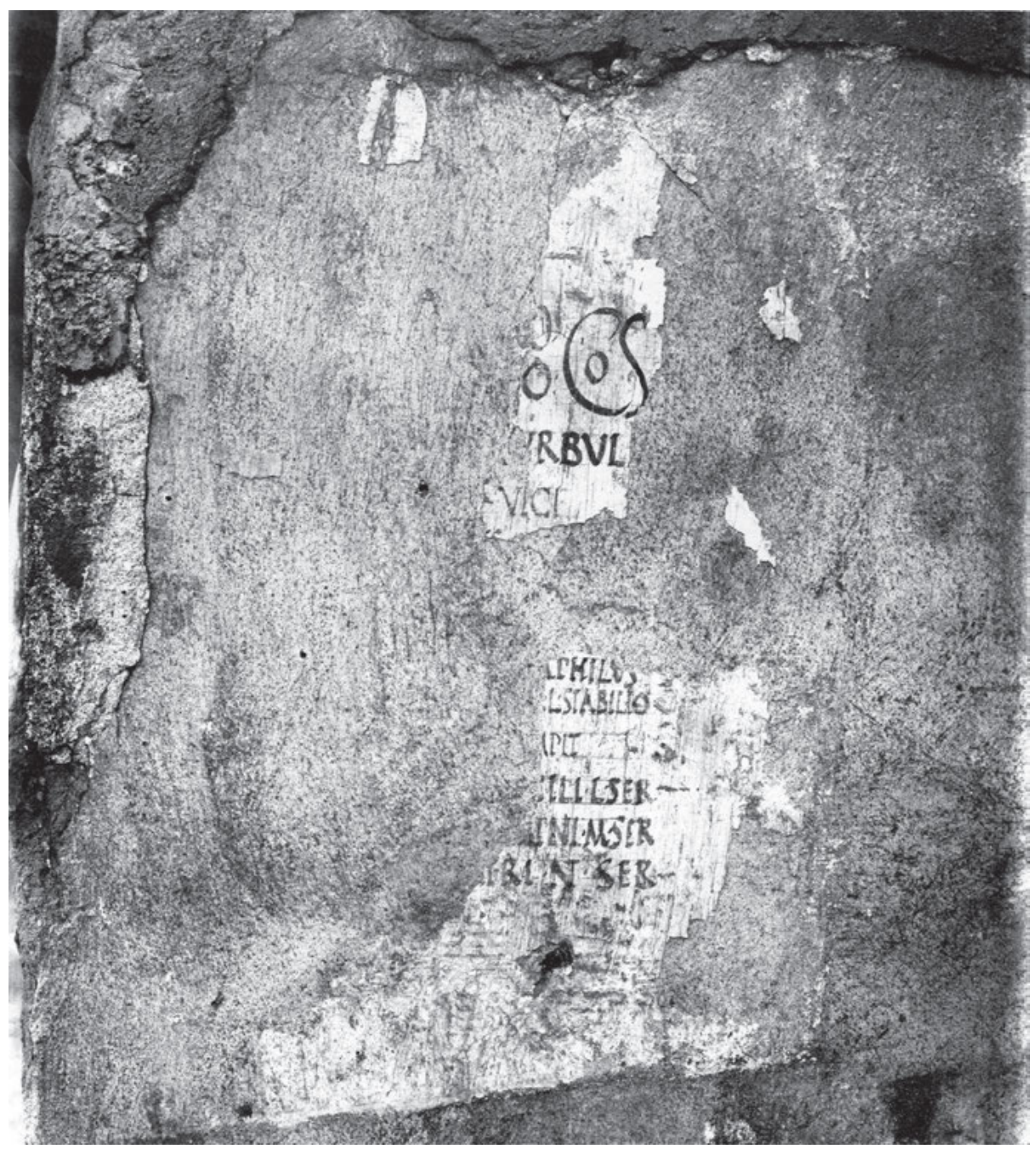

Abb. 18: CIL IV 7807 an der Südostecke von insula IX 7. ( ) Su concessione del Ministero per i Beni e le Attività Culturali e per il Turismo - Parco archeologico di Pompei. Jegliche weitere Reproduktion oder Duplikation ist untersagt.

Beamten. Der Text ist vollständig in Schwarz geschrieben und auf vier Zeilen verteilt. Auch hier sind deutlich die vorgeritzten Linien auszumachen.

Bezüglich der Topos-Inschriften am Amphitheater können lediglich anhand der CIL-Einträge Informationen über ihre Gestaltung erschlossen werden. ${ }^{313}$ Die Buchstaben waren auf weißen Putz (1096b) oder auf getünchten Tuff (1097, 1097a, 1097b)

313 CIL IV 1196. 1196a. 1196b. 1197. 1197a. 1197b. 2996. Möglicherweise auch CIL IV 1115, 1768 und 1769. 


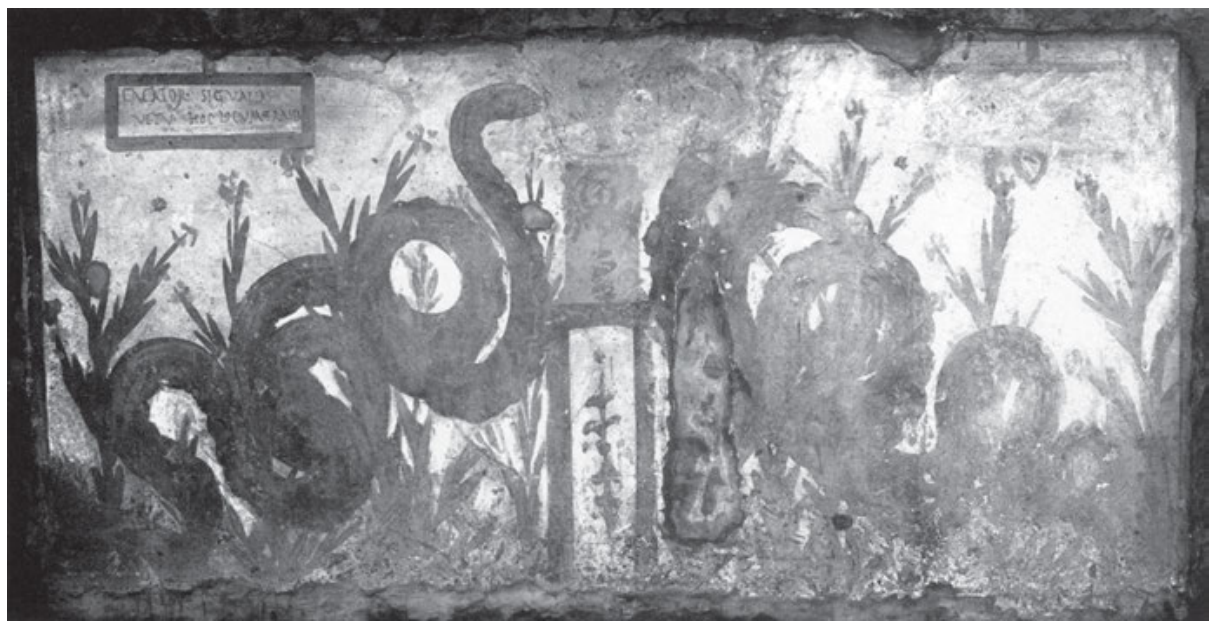

Abb. 19: Fassadenmalerei mit gemaltem Inschriftenfeld und cacator-Inschrift CIL IV 6641. (C) Su concessione del Ministero per i Beni e le Attività Culturali e per il Turismo - Parco archeologico di Pompei. Jegliche weitere Reproduktion oder Duplikation ist untersagt.

geschrieben. Drei Dipinti waren laut CIL rot (1096, 1096b, 1097b) und einer schwarz (1096b). Aber da für die übrigen ebenfalls in opere tofaceo dealbato angegeben ist, waren sie sicher auch entweder rot oder schwarz. Nur für 1096b wird die Buchstabenform angegeben: litteris quadratis.

Einige weitere Dipinti folgen nicht den beschriebenen Gestaltungsmerkmalen. Besonders hervorstechend sind darunter die sogenannten cacator cave malumInschriften CIL IV 3782, 3832, 4586, 5438, 6641, 7714, 7715 und 7716. Davon sind 4586 und 5438 mit Kohle geschrieben, also nicht eigentlich gemalt. ${ }^{314}$ Optisch sind große Unterschiede zu bemerken. Daher und auch wegen der geringen Zahl dieser Zeugnisse kann man nicht von einer typischen Gestaltungsweise sprechen. CIL IV 6641 ist zum Beispiel in eine Fassadenmalerei integriert, indem es in ein gerahmtes Feld eingeschrieben wurde (Abb.19). ${ }^{315}$ Die Buchstaben sind 2,2-2,5 cm hoch, sorgfältig und gut leserlich geschrieben, auch wenn es sich um keinen allzu geübten Schreiber handeln kann, da für die zweite Zeile die Anordnung offenbar nicht exakt geplant hatte. Auch wenn nicht genau nachgeprüft werden kann, ob diese Inschrift zeitgleich mit den Schlangen und dem Altar aufgemalt wurde, ist für sie doch der direkte Kontext von entscheidender Bedeutung, da er sehr wohl die Schutzfunktion der Schlangen

314 Vgl. Fröhlich 1991, 59 Anm.349. Inhaltlich sind diese eng verwandt mit einigen geritzten Epigrammen, besonders an Gräbern und mit Reflexen in der zeitgenössischen Literatur, vor allem Petron.71,8: praeponam enim unum ex libertis sepulcro meo custodia causa, ne in monumentum meum populus cacatum currat. Vgl. besonders Maiuri 1945, 210-211; Lebek 1976, 288; Krenkel 1963, 76. 315 cacator sic valeas / ut tu hoc locum tra<n>sea<s>; Vgl. Fröhlich 1991, 319 (F35). 


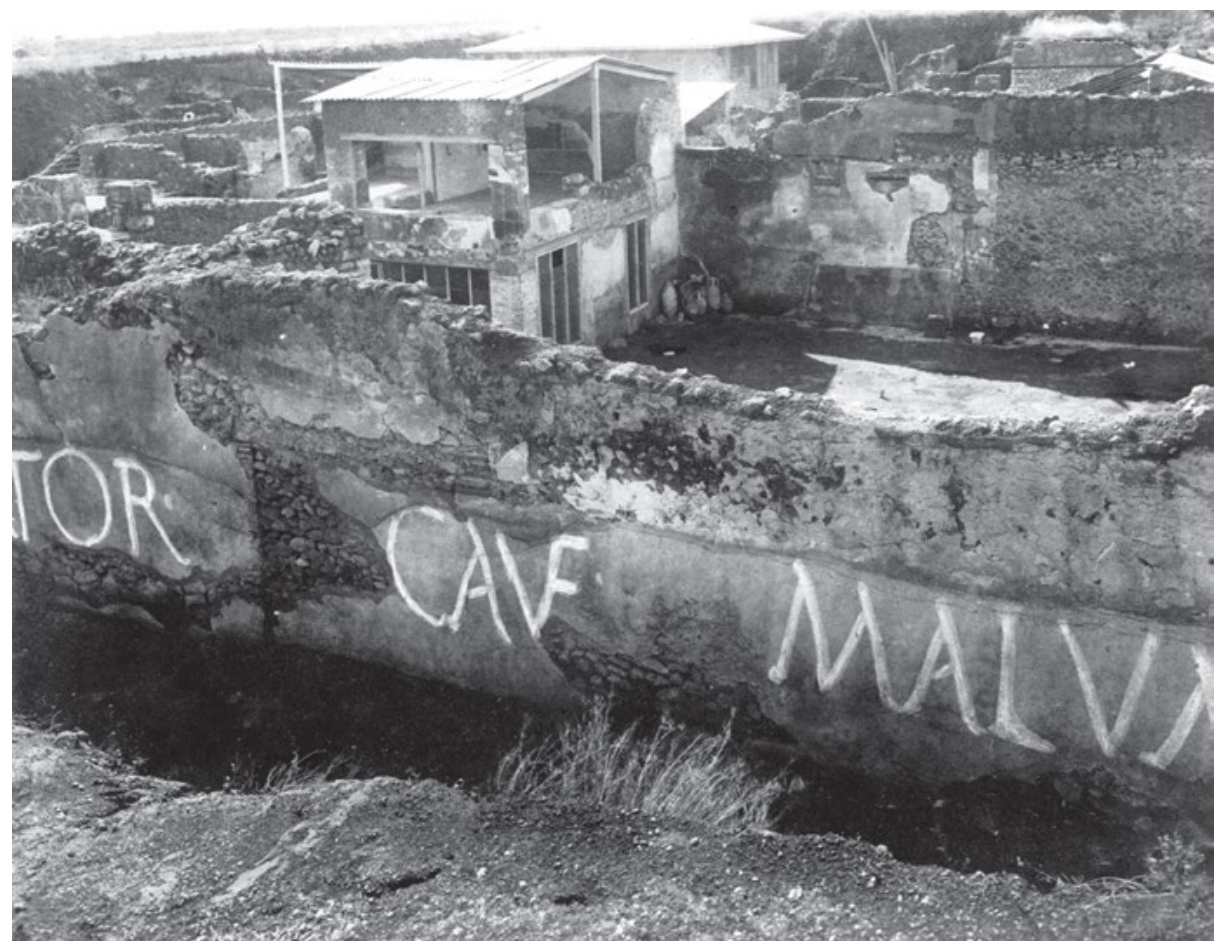

Abb. 20: Riesige cacator-Inschrift CIL IV 7715 an der Ostseite von insula III 4. (C) Su concessione del Ministero per i Beni e le Attività Culturali e per il Turismo - Parco archeologico di Pompei. Jegliche weitere Reproduktion oder Duplikation ist untersagt.

miteinbezieht. ${ }^{316}$ Sowohl der Inhalt als auch die etwas ungewöhnliche Gestaltung des Textes sprechen daher dafür, dass der Dipinto von dem Maler hinzugefügt wurde, der auch das Bild geschaffen hatte. Ganz anders erscheinen die Dipinti CIL IV 7714-7716 (Abb. 20). Sie sind in der Gasse zwischen den insulae III 4 und III 5 so aufgemalt worden, dass 7716 (an der W-Seite von III 5) den Inschriften 7714 und 7715 (an der O-Seite von III 4) gegenübersteht. Alle drei waren mit weißer Farbe (CIL: calce) auf dunklen Grund geschrieben und stechen auch durch ihre Größe hervor. ${ }^{317} 7714$ und 7715 waren beide jeweils 10,5 m lang. Die Buchstaben waren zwischen 90 und $120 \mathrm{~cm}$ hoch und die einzelnen Striche ca. $10 \mathrm{~cm}$ breit. 7716 war 7,6 m breit und weist in den drei Zeilen

316 Boyce 1942, 21; besonders auch die in diesem Zusammenhang mehrfach zitierte (z. B. Lebek 1976, 288 Anm. 3) Stelle bei Persius: Pers. 1,112-114: ,hic‘ inquis ,veto quisquam faxit oletum. pinge duos anguis: pueri, sacer est locus, extra meite: discedo.

317 Die kleine Gasse zwischen III 4 und III 5 ist heute wieder verschüttet und der Putz an den sichtbaren Flächen abgefallen. Die dunkle Färbung der Wandoberflächen ist in Varone/Stefani 2009, 273-276 zu erkennen. 

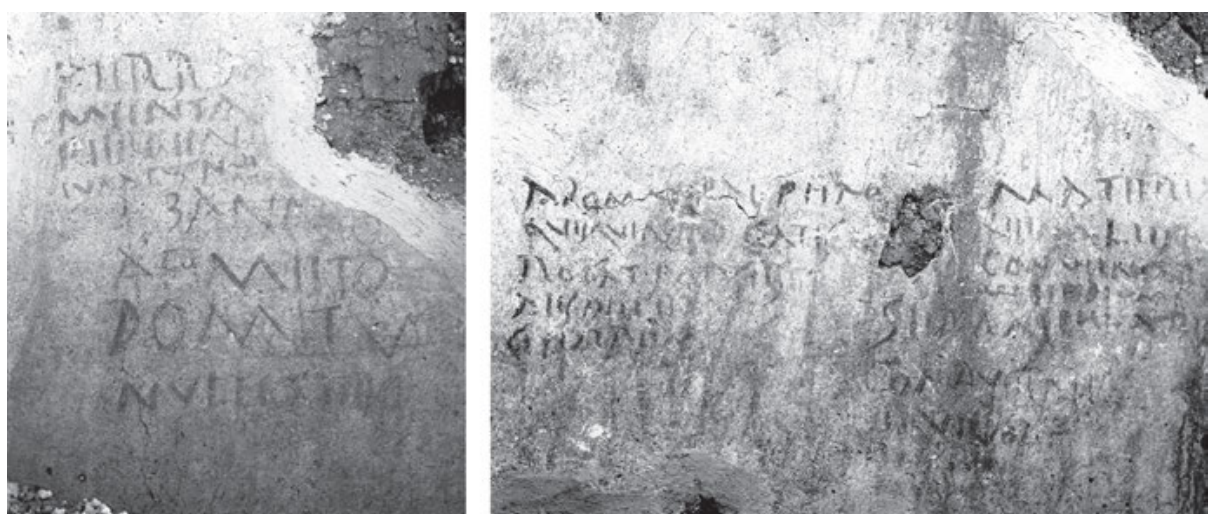

Abb. 21: CIL IV 9839a, 9839b und 9839c (von links nach rechts). 9839a befand sich östlich von Eingang I 17, 2, 9839b und $\mathrm{c}$ westlich von diesem Eingang. (C) Su concessione del Ministero per i Beni e le Attività Culturali e per il Turismo - Parco archeologico di Pompei. Jegliche weitere Reproduktion oder Duplikation ist untersagt.

Versalhöhen von 45 bis $50 \mathrm{~cm}$ auf. ${ }^{318} 7714$ und 7715 sind inhaltlich identisch (cacator cave malum). Die Buchstaben orientieren sich zwar an der actuaria, aber die einzelnen Linien sind deutlich weniger differenziert ausgeführt. Die Ligatur bei cave entspringt ganz offensichtlich nicht einem Mangel an beschreibbarer Fläche, sondern scheint ein vom Schreiber frei gewähltes Gestaltungselement zu sein. Das hier verwendete Weiß wurde ansonsten - wie bereits beschrieben ${ }^{319}$ - fast ausschließlich für die Grundierung oder das Übermalen älterer Dipinti genutzt. Gerade die Heterogenität der verschiedenen Materialien wie auch die Schriftgestaltung ist für diese Inschriften signifikant. Es handelt sich nicht um Bekanntmachungen, die wie die programmata in konzertierten Aktionen und einem Muster folgend gefertigt wurden, sondern um Einzelzeugnisse, die auf die situativ und räumlich gebundene Initiativen von Anwohnern zurückgingen. Es wurden im Stadtgebiet gerade nicht wie an Trimalchios Grab vorsorglich Verbotsschilder angebracht, sondern die Initiative war für die Betroffenen anscheinend aus gegebenem Anlass notwendig. Den Beispielen, die heute noch anhand von Abbildungen beurteilt werden können, ist eine besonders gute Leserlichkeit gemein. ${ }^{320}$ Auch dies spricht dafür, dass es sich in erster Linie um konkrete Unterlassungsauffordungen an die Mitmenschen handelte.

Einige weitere Dipinti bieten deutlich von der actuaria abweichende Formen. Dabei handelt es sich um Verkaufsangebote (CIL IV 7124), Bitten um Rückgabe eines entwendeten oder verlorenen Besitzes (CIL IV 64 und 3864 und 9948) sowie ein inhaltlich und formal auch in Pompeji einzigartiger Dipinto CIL IV 9839a-c (Abb.21), der

318 Text: cacator cave malum / aut si contempseris habeas / Iove iratum.

319 Vgl. Kap. 2.2.2.2, S.61.

320 Dies sind in Abbildungen bei Varone/Stefani 2009 die gemalten Inschriften: CIL IV 3832. 6641. 7714-7716. Die beiden Kohlezeichnungen CIL IV 4586 und 5438 sind dagegen nicht fotografisch belegt. 
am ehesten ebenfalls in den Bereich der Verkaufsangebote einzuordnen wäre. ${ }^{321}$ Bei diesen Inschriften finden sich kursive Buchstabenformen, die, ohne auf eine ebenmäBige Grundlinie zu achten, auf die Wand geschrieben wurden (besonders bei 9839a-c und 9948 (Abb. F68)). CIL IV 7124 (Abb.4) ist mit Kalkmilch geschrieben. Auch die Buchstabenformen sind hier ganz anders als bei der großen Menge der programmata.

Alle 24 überlieferten oskischen Dipinti sind im oskischen Alphabet geschrieben. ${ }^{322}$ Vetter gibt an, dass alle eítuns-Inschriften (Ve 23-28) (Abb. 2) rot waren. ${ }^{323}$ Ansonsten finden sich bei Vetter keine Beschreibungen der Gestaltung. Nicht von allen oskischen Dipinti liegen Fotografien oder apographa vor. ${ }^{324}$ In den besser dokumentierten Beispielen sind jeweils alle Striche gleich stark, das Schriftbild ist sehr stark von senkrechten, waagerechten und schrägen Strichen geprägt. Ausnahmen bilden Ve 34, ein Name, dessen zwei vorderste Buchstaben in einen nexus gesetzt waren, ${ }^{325}$ und die fünf Dipinti Ve 33 an fünf Säulen in der porticus des Popidius, in denen fünfmal das Wort vaamunim mit $38 \mathrm{~cm}$ hohen Buchstaben geschrieben war. ${ }^{326}$ Im Vergleich mit steinernen oskischen Inschriften (Abb. F30) fällt auf, dass diese sehr wohl stärker gerundete und regelmäßige Formen beinhalten. ${ }^{327}$

Sowohl bei den oskischen als auch bei den lateinischen Inschriften ist zu bemerken, dass im 1. Jh. v. Chr. ein größerer gestalterischer Unterschied zwischen gemalten und gemeißelten Texten bestand, als dies bei den tituli picti recentiores und den späteren zeitgenössischen Inschriften zu bemerken ist. Die Gestaltung von gemalten Inschriften veränderte sich innerhalb dieses Jahrhunderts hin zu stärker konventionalisierten Formen, die in einem bestimmbaren und ebenfalls auf Konventionen beruhenden Verhältnis zu den Inhalten der Texte standen.

\subsubsection{Die visuelle Wahrnehmung der Inschriften}

Steininschriften, Graffiti und Dipinti bringen durch ihre jeweiligen gestalterischen Merkmale bestimmte Voraussetzungen mit, die sich auf ihre visuelle Wahrnehmung auswirkten. Von mindestens ebenso hoher Bedeutung dafür, ob eine Inschrift gesehen oder gelesen wurde oder eben nicht, sind jedoch auch ihre Lokalisierung im Kontext sowie das Verhalten der potentiellen Betrachter. Hierbei sind vor allem die Faktoren der Aufmerksamkeit und der Bewegungsgeschwindigkeit bzw. der Ruhe von

$321 \mathrm{Zu}$ Lesung dieser Inschrift vgl. v. a. Solin 1973b, 275, der hier signifikant von Della Cortes Deutung abweicht.

322 Vgl. auch die Rekonstruktion bei Zvetaieff 1878 Taf. 15.

323 Vetter 1953, 54.

324 Della Corte 1916b, 158; Varone/Stefani 2009: Ve 24 S.317; Ve 27 S. 368; Ve 28 S. 257-259; Ve 30 S. 377; Ve 28 S. 54.

325 Vetter 1953, 60; Della Corte 1916b, 158.

326 Vetter 1953, 59.

327 Dabei sticht vor allem die besonders akkurat gearbeitete Stifterinschrift des V. Aadirans hervor: Cooley 2002, 79 Abb. 6.1. 
Bedeutung. Im Folgenden soll anhand von Theorien und Erkenntnissen der Wahrnehmungspsychologie versucht werden, sich der Frage anzunähern, wie die Dipinti wahrgenommen wurden. Manche dieser Überlegungen sind auch auf Graffiti und Steininschriften übertragbar, während in anderen Fällen zu viele Faktoren unbekannt bleiben.

Durch die beschriebenen Merkmale der Dipinti erreichten diese eine hohe visuelle Präsenz, die nicht nur inhaltliche Aspekte, sondern vor allem auch ästhetische Faktoren betrifft. Die Kombination von roter oder schwarzer Farbe auf hellem Untergrund sorgte einerseits für einen hohen Kontrast und gute Sichtbarkeit. Andererseits waren die Dipinti sich untereinander ähnlich und fügten sich mit ihrer roten und schwarzen Gestaltung in das Farbspektrum ihrer Umgebung ein. Folglich waren sie zwar gut zu sehen und zu lesen, stellten aber keine Durchbrechung eines ansonsten konsistenten und visuell harmonischen Umfeldes dar. Wie an den Fallbeispielen in Kapitel 4 deutlich wird, ist im städtischen Raum damit zu rechnen, dass Betrachter und potentielle Leser mit ganz unterschiedlichen Voraussetzungen und in unterschiedlichen Situationen mit den Dipinti konfrontiert waren. Wie wirkten sich also die gestalterischen und materiellen Merkmale der Dipinti auf ihre sinnliche Wahrnehmung aus?

Für die visuelle Wahrnehmung und die kognitive Verarbeitung spielen mehrere komplexe Faktoren eine Rolle, die in unterschiedlicher Weise zusammenwirken. Prinzipiell sind dies einerseits Merkmale dessen, was zu sehen ist und andererseits die beim Betrachter gegebenen Voraussetzungen. Die wahrnehmungspsychologischen Erkenntnisse und Ansätze, welche sich mit der visuellen Objektwahrnehmung befassen, haben sich seit Max Wertheimers Gestaltpsychologie weiterentwickelt und besonders anfänglich formulierte allzu optimistische Annahmen wurden inzwischen vorsichtig revidiert. Grundlegende Fragen der Wahrnehmungspsychologie hinsichtlich der Objektwahrnehmung, die in unserem Kontext sinnvoll gestellt werden können, betreffen die Organisation kleiner Elemente (von Seiten des Betrachters) zu einem größeren Ganzen, das Erkennen und Deuten von Gegenständen wie auch die Unterscheidung zwischen einem Gegenstand und dem Hintergrund. ${ }^{328}$ Erkenntnistheoretisch problematisch ist, dass die in einem städtischen Kontext verorteten und zugleich mit unzähligen weiteren Reizen konkurrierenden Wandinschriften im Grunde ein zu komplexes Untersuchungsobjekt darstellen. Schon in der Gestaltpsychologie wurden mit ihren Gesetzen prägnante und doch ganz simple Grundsätze formuliert, die jeweils von modellhaften, idealen Anordnungen ausgingen. ${ }^{329}$ In aktuellen Studien zur visuellen Wahrnehmung werden noch viel kleinteiligere Fragestellungen formuliert und

328 Wertheimer 1912, Formulierung der „Faktoren“ (später „Gesetze“): Wertheimer 1923, zur Annahme, dass der Mensch bemüht ist, bei einer Vielzahl von Reizen, zusammengehörige Reize zusammenzufassen und als Gestalt wahrzunehmen: 302; Vgl. Goldstein 1997, 163-203.

329 Wichtig ist hier auch Rudolf Arnheims Verknüpfung dieser Gesetze mit der Betrachtung von Bildwerken, welche jedoch im Vergleich mit den hier behandelten Wandinschriften noch viel stärker von ihren Urhebern konstruiert wurden: Arnheim 1965, 29-67. 
in Experimenten an Probanden empirisch untersucht, um zu kontrollierbaren Ergebnissen zu gelangen. Eine Anwendung auf die pompejanischen Wandinschriften kann daher lediglich bestimmte Möglichkeiten aufzeigen, ohne dass diese an modernen, geschweige denn antiken Subjekten verifiziert werden könnten.

Von den in der Gestaltpsychologie formulierten Gesetzen kommen bei der Wahrnehmung der Dipinti besonders das Gesetz der Nähe und das Gesetz der Ähnlichkeit zum Tragen. Die Buchstaben der actuaria sind derart gelängt, dass sie sehr dicht nebeneinander platziert werden konnten und bei relativ großer Buchstabenhöhe doch keine übermäßig große Breite des Gesamttextes zur Folge hatten. Zudem werden sich die einzelnen Buchstaben durch die wiederkehrende Betonung der senkrechten Hasten ähnlich, sodass die Inschriften als ein kompaktes Ganzes erscheinen. Die Figur-Grund-Trennung führt darüber hinaus zu einer Fokussierung auf die als Figur erkannten Wandinschriften im Gegensatz zum undefinierten Grund. Das Gesetz der Nähe und der Ähnlichkeit kommt zum Tragen, wenn Dipinti gezielt dicht beieinander platziert wurden, was häufig geschah, wenn inhaltliche Bezüge bestanden. Durch die Nähe sind Betrachter geneigt, Einzelelemente als zusammengehörig zu verstehen, ohne sie bereits eingehender betrachtet zu haben.

Die vorangehenden Beobachtungen könnte auch ein Betrachter machen, der nicht lesen kann, oder der einer Fassade nur kurze Aufmerksamkeit schenkt. Was geschah aber, wenn ein Passant oder eiliger Verkehrsteilnehmer gar keine Aufmerksamkeit aufbringen wollte oder konnte, etwa, weil er sich sehr schnell bewegte oder weil zu viele Dinge und Vorgänge um ihn herum zugleich als Reize auf ihn einstürmten? Die Wahrnehmungspsychologie fragt seit den 1980ern vermehrt danach, in welchen Stufen Reize verarbeitet wurden. Für die Frage nach dem eiligen Passanten ist vor allem die präattentive Verarbeitung von Bedeutung. Damit ist gemeint, dass ein Reiz nur so kurz verfügbar ist, dass der Betrachter nicht in der Lage ist, die Aufmerksamkeit bewusst auf einen bestimmten Bereich oder ein bestimmtes Merkmal zu richten. Bestimmte Informationen werden aber dennoch verarbeitet, auch ohne dass der Betrachter dessen gewahr werden muss. ${ }^{330}$ Eine solche Situation ist der unseres Passanten vergleichbar. ${ }^{331}$ In entsprechenden Versuchen stellte sich heraus, dass einfache Merkmale wie Größe, Farbe, Dichte, Länge, Farbintensität und Orientierung der präattentiven Wahrnehmung zugänglich sind. ${ }^{332}$ Dies sind Merkmale, die auch von den Dipinti-Malern genutzt wurden, sowohl um die einzelne Inschrift von ihrer Umgebung

330 Ein geeigneter kurzer Reiz dauert ca. 160 bis 250 Millisekunden oder noch kürzer. Vgl. allgemein zur präattentiven Wahrnehmung https://www.csc2.ncsu.edu/faculty/healey/PP/ (Stand: 1.7.2020). Einen guten Überblick bietet: Goldstein 1997, 184-189.

331 Mit der besonderen Einschränkung, dass in den psychologischen Studien die Teilnehmer wissen, dass sie an einer Studie teilnehmen.

332 Vgl. https://www.csc2.ncsu.edu/faculty/healey/PP/ (Stand: 1.7.2020) mit Literaturverweisen, besonders jeweils die Studien zu einzelnen dieser Merkmale: Julész/Bergen 1983, Treisman 1985, Treisman/Gormican 1988, Healey/Enns 1999. 
abzuheben, als auch, um sie darin einzufügen. Wenn alle Dipinti an einer Fassade rot waren, konnte ein einzelnes schwarz geschriebenes Wort bereits Aufmerksamkeit bewirken, ohne dass damit eine besondere inhaltliche Botschaft verknüpft gewesen sein muss. ${ }^{333}$ Die präattentive Wahrnehmung der Dichte führt vergleichbar mit dem Gesetz der Nähe in der Gestaltpsychologie dazu, dass ein Dipinto als Einheit aufgefasst werden konnte. Die Farbintensität der Dipinti verringerte sich im Laufe der Jahre, sodass es leicht sichtbar war, wenn eine frische, in kräftiger Farbe gemalte Inschrift hinzukam. Ein flüchtiger, vorbeieilender Betrachter konnte demnach nicht nur überhaupt wahrnehmen, dass eine Wand beschrieben war, sondern auch, dass es sich um mehrere Inschriften mit teilweise spezifischen Merkmalen handelte. In einer 2016 erschienenen Studie konnte darüber hinaus gezeigt werden, dass ein Objekt innerhalb einer Fläche (präattentiv) eher gefunden wird, wenn die umgebenden Strukturen eine Art Rahmen bildeten. ${ }^{334}$ Dies bedeutet mit Blick auf die Dipinti, dass die geweißelten Flächen und tabulae ansatae, in die viele programmata eingeschrieben waren, nicht bloß als Hintergrund zur Erzeugung eines Kontrastes dienten, sondern auch dazu führten, dass die betreffenden Wahlwerbungen eher ins Auge sprangen, als solche, die auf einen undifferenzierten Untergrund aufgemalt waren.

Es ist selbstverständlich nicht anzunehmen, dass die Maler diese Prinzipien bewusst mit dem Ziel anwendeten, bestimmte Wahrnehmungsmechanismen in Gang zu setzen. Dennoch lässt sich aus diesen Überlegungen schließen, dass für die Dipinti, die in klaren Formen, einfachen Farben und nach bestimmten Gestaltungsmustern angelegt waren, die Chancen, dass sie gesehen wurden, relativ gut waren. Dies gilt gerade auch im Vergleich zu komplexeren Wandmalereien und Fassadenbildern, die häufig kleinteiliger und vielschichtiger waren. Das Lesen und die zielgerichtete Fokussierung und Wahrnehmung einer einzelnen Wandinschrift folgten wenn überhaupt jedoch erst im nächsten Schritt. Für Betrachter, die des Lesens unkundig waren oder die sich nicht die Zeit nahmen, sie zu lesen, boten die Dipinti trotzdem zahlreiche Informationen, die eine Kategorisierung und eine inhaltliche Einordnung ermöglichten.

\subsubsection{Zwischenergebnis}

Anhand der zahlreichen erhaltenen bzw. dokumentierten Dipinti in Pompeji und Herculaneum zeigt sich deutlich, dass sich die Schreiber und die Betrachter einer Reihe von Merkmalen gegenübersahen, die im Anbringungsvorgang, in Konventionen oder in ästhetischen Absichten begründet waren. Dieses Spektrum ist jedoch nicht durch willkürliche Praktiken im Umgang mit Texten und Inhalten zu erklären, sondern war ganz offensichtlich mit etablierten Gestaltungsmustern für bestimmte Typen von Inschriften korreliert. Dies ist vor allem bei den programmata recentiora

333 Vgl. die schwarz geschriebenen programmata an der Fassade der Casa di Trebius Valens: S.200. 334 Kimchi et al. 2016. 
und den edicta munerum nicht von der Hand zu weisen. Bei den meisten dieser Zeugnisse sind wiederkehrende Schemata festzustellen. Gut zu beobachtende Parameter sind die Buchstabengröße, deren Variation innerhalb der Inschrift, die Buchstabenformen und wiederum verschiedenen Ausprägungen im Rahmen eines Dipintos, aber auch der Einsatz verschiedener Farben und die durch vorgeritzte Linien oder auffällige Symmetrien nachvollziehbare ordinatio. Umso stärker fallen daher Ausnahmen auf, die genau in diesen Merkmalen von dem Gewohnten abweichen.

Gerade im Hinblick auf Rezeptionspraktiken, aber auch auf Bewegungsabläufe ist es daher von Bedeutung, dass vor allem bei den programmata und den edicta munerum die Form und Gestaltung offensichtlich so konsistente Gestaltungsmuster aufweisen, dass auch die Form zum Bedeutungsträger wurde. Diese Bedeutungen konnten selbstverständlich erst dadurch zugeschrieben werden, dass sich die Form als typisch etablierte. Die Formen sind jedoch nicht erstarrt, sondern lassen auch Gestaltungsspielräume zu.

Mouritsen betont den ephemeren Charakter der Aufschriften und nimmt an, dass man bewusst flüssigere Farbe als Schreibmaterial für Dipinti wählte, die nicht für eine lange Dauer sichtbar sein mussten. ${ }^{335}$ Sabbatini Tumolesi geht hingegen vor allem mit Bezug auf die zweite größere Gruppe, die edicta munerum, welche ebenfalls inhaltlich vordergründig auf ein einmaliges Ereignis zu beziehen sind, davon aus, dass diese sich trotz negativer Umwelteinflüsse und der Überlagerung durch jüngere Farbschichten seit augusteischer Zeit erhalten hatten. ${ }^{336}$ Tatsächlich zeigt die Erhaltung zahlreicher Dipinti seit nunmehr einigen Jahrzehnten nach ihrer Freilegung, dass diese ähnlich haltbar sind wie Wandmalereien.

\subsubsection{Graffiti}

\subsubsection{Textträger}

Graffiti wurden auf die gleichen Beschreibflächen geschrieben und gezeichnet wie Dipinti, nämlich vor allem auf Putz und Stein an Fassaden, Säulen und Wänden im Innen- und Außenbereich verschiedenster Gebäude. ${ }^{337}$ Anstatt ein bestimmtes Material wie flüssige Farbe auf die Oberfläche aufzutragen, ritzt man die Oberfläche mit einem spitzen Gegenstand an. Der visuelle Effekt wird daher entweder durch einen Farbkontrast zwischen der Oberfläche des Putzes und der unmittelbar darunter liegenden Schicht erzielt (Abb. F31), oder - wie auch bei in Stein gehauenen

335 Mouritsen 1988, 31: „The electoral notices are painted, and therefore ephemeral, inscriptions“. So sieht es auch Angela Donati: Donati 1998, 99-100.

336 Sabbatini Tumolesi 1980, 113.

337 Geritzte Inschriften und Zeichnungen auf beweglichen Objekten, wie Gefäßen, Ziegeln, Scherben oder Tafeln werden hier nicht behandelt. Vgl. dazu Solin 2008, 100. 
Inschriften - dadurch, dass je nach Einfallswinkel des Lichtes unterschiedliche Flächen der eingeritzten Furche verschattet und damit deutlich sichtbar sind (Abb. F32). ${ }^{338}$

Die Mehrheit der Graffiti wurde auf Putz geschrieben, doch es finden sich auch einige Befunde, in denen die Oberfläche der verbauten Steinblöcke als Beschreibfläche diente. ${ }^{339}$ In Pompeji zählen dazu die Säulen im atrium und im Peristyl der Casa di Obellius Firmus (IX 14, 1-4) ${ }^{340}$ sowie im Außenbereich die Fassade von VI 8, 21, ${ }^{341}$ die SW-Ecke von insula VI $12^{342}$ und mehrere Pfeiler zwischen Taberneneingängen an der Via dell'Abbondanza. ${ }^{343}$ Die Steinfassaden, die Graffiti tragen, bestehen alle aus dem bräunlich-gelben Tuff, der sich durch sein geringes Gewicht wie auch durch seine geringe Härte als leicht zu bearbeitendes Material auszeichnet. Zum Schreiben oder Zeichnen auf diesem Stein eigneten sich daher wie bei Putz ebenfalls fast alle ausreichend spitzen und stabilen Gegenstände. Anders als bei Putz konnten Graffitischreiber allerdings bei Stein nicht mit den unterschiedlichen Färbungen der einzelnen Schichten spielen. Die Wirkung der Steingraffiti beruht deshalb weniger auf einem farblichen Kontrast, als auf der Licht- und Schattenwirkung der angeritzten Oberfläche.

Die üblichste Anbringung eines Graffito war jedoch, ihn in den trockenen Putz zu ritzen. Charakteristisch für diese Graffiti sind ausgerissene Kanten an den Rändern der eingeritzten Linien, die je nach Körnung des Putzes und je nach Strichdicke unterschiedlich weit ausgreifen (Abb.22. 23). Bemalte Wände waren mit einem Feinputz versehen. ${ }^{344}$ Das gilt auch für einen Großteil der Außenwände, die ebenfalls oft bemalt waren, doch gerade in den Nebenstraßen waren große Flächen nur mit einem groben Cocciopesto-Putz verkleidet. ${ }^{345}$

Das Schreiben in den trockenen Putz vollzieht sich als sekundäre Nutzung der Wandflächen. ${ }^{346}$ Es gibt keine Hinweise darauf, dass die Möglichkeit, dass jemand ein Feld als Fläche zum Schreiben oder Zeichnen eines Graffito verwenden könnte, im Vorfeld berücksichtigt wurde - im Gegensatz zur Wandmalerei, deren Gestaltung technisch und hinsichtlich der Arbeitsschritte mit der Anbringung der schützenden

338 Vgl. zu den Graffitizeichnungen in den fauces Casa del Menandro Langner 2001, Taf. 159 Nr. 2482. 339 Langner zu den Zeichnungen: Langner 2001, 13 mit Anm.13.

340 CIL IV 8961-8963. 9300.

341 CIL IV 1269. 4431-4433.

342 CIL IV 1372.

343 Zwischen VII 1, 11 und 12: CIL IV 2128-2131; zwischen VIII 5, 7 und 8: CIL IV 2043; zwischen VI 8, 21 und 22: CIL IV 2044. In Herculaneum finden sich ebenfalls Graffiti auf Stein, die allerdings nicht publiziert sind.

344 Als oberste Schichten erwähnt Vitruv mit Marmormehl angerührte Mischungen, denen Zuschläge von immer feinerer Korngröße beigemengt wurden: Vitr. 7,3,6. Klinkert ergänzt dies um die Beobachtung, dass auch die Dicke dieser Schichten stark abnimmt: Klinkert 1957, 125.

345 Im CIL finden sich als Beschreibung solcher Oberflächen die Begriffe rubro tectorio oder in signino. Vgl. dazu Kapitel 2.2.2.1.

346 So auch Langner 2001, 12 und Chaniotis 2011, 193. 



Abb. 22: Ve $64 \mathrm{im}$ Durchgang der Porta di Nola. (C) Su concessione del Ministero per i Beni e le Attività Culturali e per il Turismo Parco archeologico di Pompei. Jegliche weitere Reproduktion oder Duplikation ist untersagt.

Abb. 23: CIL IV 10224 im Tonnengewölbe des Grabes EN 6. (C) Su concessione del Ministero per i Beni e le Attività Culturali e per il Turismo Parco archeologico di Pompei. Jegliche weitere Reproduktion oder Duplikation ist untersagt.

Putzschichten verknüpft ist. Die Graffiti ihrerseits nehmen oft Bezug auf bereits vorhandene Gestaltungselemente, Schemata und Einzelmotive der Malereien. ${ }^{347}$ Auf Putzplatten, die aus der Wandverkleidung der basilica entfernt wurden und heute im Museum in Neapel lagern, finden sich Beispiele, in denen der Schreiber die Buchstaben in die Bänder zwischen den stuckierten Quadern eingeschrieben hat, sodass sie beinahe deren ganze Höhe einnehmen (Abb.F33). ${ }^{348}$ Einen inhaltlichen Bezug zu einem aufgemalten Larenbild an der NW-Ecke der insula I 11 weist CIL IV 8426 auf:

347 Z. B. wurden in cubiculum 14 in der Casa di Obellius Firmus (IX 14, 1-4) Graffitizeichnungen in die schwarzen Felder hinein gezeichnet und kommen so hervorragend zur Geltung. Vgl. dazu: Voegtle 2012, 108-111.

348 Vgl. CIL IV Hauptband S. 113. Auch: CIL IV 1833 und 1935; Vgl. Varone 2012, 355. 356. 
Lares sanctos / rogo te ut war in Rot links neben dem Bild auf der Höhe von Kopf und Arm eines der beiden Laren geschrieben. ${ }^{349}$

Abgesehen davon scheinen sich die Graffitischreiber für die Ausrichtung und Platzierung der Texte ähnlich wie die scriptores der Dipinti an der horizontalen Unterteilung der Wand orientiert zu haben, wie auf den Flächen nördlich und südlich von Eingang I 13, 9 zu erkennen ist (Abb. F34). Dort wurden vor allem in der unteren Zone viele Graffiti (Langner 42. 46. 1076; CIL IV 10070-10075) parallel oder zumindest in deutlicher Orientierung zur Oberkante des unteren Fassadenbereiches in den Cocciopesto-Putz geritzt, während im oberen Bereich eine ganze Reihe von programmata zu lesen war. ${ }^{350}$ Der Putz in der oberen Zone ist viel gröber als im unteren Bereich. Die kleineren Graffitibuchstaben und Einzelmotive wären in dieser Oberflächenstruktur nicht zu erkennen gewesen. An zahlreichen weiteren Wänden, die eine Einteilung in zwei Zonen aufweisen, lässt sich ebenfalls beobachten, dass die Graffiti eher in den unteren, feiner geglätteten Bereich geschrieben wurden. ${ }^{351}$

Eine kleine Anzahl an Graffiti in Pompeji und Herculaneum scheint in den noch feuchten Putz geritzt worden zu sein. ${ }^{352}$ Diese finden sich in Herculaneum im Frauentrakt der Therme (VI 1-10) ) $^{353}$ und in Pompeji im lupanar (VII 12, 18.19) ${ }^{354}$, den Stabianer Thermen (VII 1, 8.14.15.16.17.48.49.50.51) ${ }^{355}$ und $\mathrm{zu}$ beiden Seiten des Eingangs II 1, 10. ${ }^{356}$ Als Beispiel zur Verdeutlichung der Merkmale soll hier der Graffito aus dem Frauenbad in Herculaneum dienen (Abb.F35). ${ }^{357}$ Die Buchstaben sind sehr klar und deutlich zu erkennen. Die Linien dieses Graffito sind mit gleichmäßigem Druck und in weicher Rundung gezogen, wie es beim Ritzen in den spröden trockenen Putz nicht zu erreichen wäre. An den Kanten der Linien ist der Putz aufgeworfen statt abgeplatzt, und wo der Schreiber die Linie begonnen hat, ist an einer kleinen Verbreiterung, besonders deutlich beim C, zu erkennen, wie sich der Griffel in den weichen Untergrund gedrückt hat. Der Schreiber rutscht nicht ab, was darauf hindeutet,

349 Der Graffito ist nicht erhalten. Siehe dazu: Fröhlich 1991, 308-309 (F7); Varone/Stefani 2009, 134-135; Fröhlich nennt roten Stein als Schreibgerät, laut CIL ist er litteris gypso subrubro scriptis.

350 CIL IV 9860-9866. 9868-9871.

351 Dieses Bild und insbesondere die Verteilung von Dipinti im oberen Bereich und Graffiti im unteren Bereich der Fassaden finden sich unter anderem an der Fassade der Casa del Menandro (I 10, 4) (CIL IV 8285-8309) und an der Casa di Paquius Proculus (I 7, 1) (CIL IV 8060-8066) wieder. Bei beiden Häusern war die Fassade im unteren Bereich mit einem Feinputz versehen, geglättet und rot bzw. schwarz bemalt, sodass sich durch die Ritzung ein deutlicher Kontrast ergab.

352 Zu den in den feuchten Putz geritzten Graffiti siehe auch: Lohmann 2018, 250; 253 Abb. 89.90.

353 In Korridor N: CIL IV 10603, Abb.: Varone 2012, 498 oben.

354 In den Räumen b, c und f sowie auf dem Wandstück zwischen den Räumen c und d: Raum b: CIL IV 2290, Abb. Varone 2012, 323; Raum c: CIL IV 2284. 2285. 2288, Abb. Varone 2012, 324-325; Raum f: CIL IV 2183. 2225. 2227a. 2231, Abb. Varone 2012, 341. 333. 331.

355 In Korridor K: CIL IV 2116, Abb. Varone 2012, 299 unten rechts.

356 CIL IV 10082-10099 und Langner 1082 Abb. Varone 2012, 94-101; Langner 2001 Taf. 62. Zudem findet sich zu CIL IV 10030 im CIL der Hinweis: litteris cuspide lignea in tectorio molli diligenter ductis. 357 Della Corte bzw. Ciprotti vermerkt bei diesem Graffito: in podio signino molli. 
dass der Untergrund noch nicht besonders fest war und er wenig Kraft aufwenden musste. Ähnliches lässt sich in den anderen genannten Kontexten beobachten. Der Querschnitt der Linien von CIL IV 2183 aus dem lupanar (Abb.F36) läuft nicht unten spitz zu, sondern ist flacher, was auf ein stumpfes Schreibgerät hindeutet. ${ }^{358}$

Auch für die Graffiti boten sich den antiken Schreibern also nahezu unbegrenzte Beschreibflächen, nämlich ebenso wie für die Dipinti sämtliche Wände im öffentlichen Raum, Säulen, freistehende Monumente und - anders als für die Dipinti - vor allem auch die Wände im Inneren der Gebäude. Die wichtigsten Voraussetzungen bestanden darin, dass die Oberfläche stabil und nicht übermäßig porös sein durfte und dass sie mit einem entsprechenden Gerät einzuritzen war. Allerdings spielten für die Graffitischreiber andere Faktoren eine Rolle als für die Dipintimaler. Dies spiegelt sich in den Anbringungsstellen der Inschriften wider.

\subsubsection{Verwendete Materialien und Werkzeuge}

Unter Graffiti werden in der Archäologie und Epigraphik Inschriften und Zeichnungen verstanden, die mit einem spitzen Gegenstand in eine harte Oberfläche geritzt wurden. ${ }^{359}$ Hinzugezählt werden meist aber auch textuelle Graffiti und Zeichnungen, die mit Rötel, Stein, Kreide, Tinte oder Kohle angefertigt wurden, auch wenn der Prozess technisch anders ist. ${ }^{360}$ Das trifft ebenso auf Bleistiftzeichnungen $\mathrm{zu}^{361}$ Die drei bisher erschienenen CIL-Bände, die die pompejanischen und herculanensischen Graffiti behandeln, liefern uneinheitliche und damit schwierig zu vergleichende Angaben zu den Schreibgeräten und Schreibmaterialien. Im Hauptband (Zangemeister) finden sich unter der Überschrift graphio scripta nur solche Inschriften, quae graphii sive stili aut alius cuiuslibet rei cuspide incisae vel potius scariphatae sunt. ${ }^{362}$ Alle Exemplare, quae carbone vel terra aliqua facta sunt, sind dagegen unter den tituli picti aufgeführt, da sie genau genommen einen Farbauftrag und keine Ritzung erzeugen. ${ }^{363}$

358 Die Tatsache, dass in noch feuchten, weichen Putz geschrieben wurde, und besonders die vielen Graffiti in Raum f des lupanars weisen auf weitere interessante Umstände hin: Zum einen trocknete der Putz offensichtlich langsam genug, dass eine ganze Reihe von Personen - der Lokalität entsprechend sicherlich nacheinander - Graffiti hineinritzen konnten. Leider bieten die Graffiti keine Anhaltspunkte dafür, in welcher Zeitspanne der Putz noch derart formbar war, was auch mit Blick auf die Technik der Wandmalerei interessant wäre. Vgl. Allag/Barbet 1972, 1058. Zum anderen stellte es für die Betreiber anscheinend kein Problem dar, den Betrieb fortzusetzen oder nach Renovierungsarbeiten wiederaufzunehmen, bevor die Wandverkleidung fertig ausgehärtet war. Auch die Inhalte der Graffiti deuten darauf hin, dass das lupanar bereits wieder in Betrieb war.

359 Langner 2001, 13.

360 Diese haben sich nur selten erhalten, sind jedoch im CIL mit Hinweisen auf das Material wie carbone oder lapide rubro gekennzeichnet. Vgl. Langner 2001, 12-13 mit Anm. 8.

361 Bei einer Zeichnung eines Gladiators in Grab EN 12 in der Nekropole an der Porta di Nocera muss es sich um eine Bleistiftzeichnung handeln (Langner 805). Anders: Langner 2001, 13 Anm. 8.

362 Vgl. CIL IV Hauptband S. 76.

363 Vgl. CIL IV Hauptband S. 7. 
In Supplement 2 (Mau und Zangemeister) und Supplement 3 (Della Corte und Ciprotti) erscheinen Schreibmaterialien wie Kohle, Rötel oder Steine dagegen unter den graphio scripta. Diese Schreibgeräte werden dort in den Scheden nur dann angegeben, wenn es sich nicht um geritzte Inschriften handelt oder andere Auffälligkeiten hervorgehoben werden sollen. ${ }^{364}$

In Tabelle 1 sind die Angaben, die sich im CIL zu den Schreibgeräten finden, zusammengestellt. ${ }^{365}$ Diese beziehen sich natürlich nur auf die textuellen Graffiti. Aufgrund der Vergänglichkeit dieser Schreibmaterialien können die Angaben nur in wenigen Fällen überprüft werden. $\mathrm{Zu}$ einigen der Kohlegraffiti bietet Varone Fotografien. ${ }^{366}$ Besonders deutlich offenbart sich der unterschiedliche Einfluss der Dokumentationsweise von Zangemeister, Mau, Della Corte und Ciprotti beim Schreibstoff Kohle, wie ein Vergleich der jeweiligen Anzahl der Kohlegraffiti in den verschiedenen CIL-Bänden aufschlussreich (Tabelle 2) zeigt. Im Hauptband sind weniger als $1 \%$ der Graffiti als Kohlegraffiti gekennzeichnet, sodass der Anschein erweckt wird, in den regiones VI, VII und VIII, die dort vor allem erfasst sind, habe es viel weniger Kohlegraffiti gegeben als in den später ausgegrabenen Bereichen der Stadt. Für Herculaneum, das von Ciprotti unter Zuhilfenahme von Della Cortes Notizen behandelt wurde, ist der Anteil der Kohlegraffiti an der Gesamtmenge dagegen besonders hoch. Diese Unterschiede sind vermutlich sowohl auf den Erhaltungszustand als auch auf die Arbeitsweise bei der Dokumentation der Wandinschriften zurückzuführen. Während sich Zangemeister im Hauptband oft auf Berichte und Beschreibungen anderer verlassen musste und die Entdeckung der Wandinschriften in vielen Fällen schon Jahrzehnte zurücklag, wurde die Dokumentation der Schriftzeugnisse für Supplement 2 und Supplement 3 meist von den Editoren selbst und in engem zeitlichem Zusammenhang zu den Grabungen durchgeführt. Daher ist zum einen damit zu rechnen, dass auch in den westlichen Stadtbereichen mehr Kohlegraffiti zu finden gewesen wären, und zum anderen, dass dies möglicherweise auch für andere Schreibmaterialien wie Stein, Kreide oder Tinte gilt.

364 Vgl. CIL IV Suppl. 2 S. IV; in CIL IV Suppl. 3 finden sich keine Vorworte zu den einzelnen Abschnitten, aber unter den Scheden finden sich zahlreiche Hinweise auf die Beschaffenheit der Schreibgeräte. In Supplement 2 und 3 wird auch eine kleine Anzahl von gemalten Inschriften unter den Graffiti aufgeführt, wie z. B. CIL IV 10229b (Abb. F46). Sie ist mit der gleichen flüssigen Farbe und einem Pinsel gemalt wie die Dipinti, doch die Buchstabenformen entsprechen nicht der actuaria und der Text beinhaltet nur einen Namen. Während die Inschrift also technisch als Dipinto zu bezeichnen ist, ist sie nach dem Inhalt im Kontext der Graffiti zu sehen. Auch der Anbringungsort im Inneren des Gewölbes eines Grabmals macht ihn eher mit Graffiti vergleichbar.

365 Darunter sind auch 518 Graffiti aus Boscoreale, Boscotrecase, Scafati, Stabiae und Neapel.

366 Bei CIL IV 8213 ergab seine Autopsie, dass es sich nicht, wie im CIL angegeben, um einen Kohlegraffito, sondern um eine geritzte Inschrift handelt. Vgl. Varone 2012, 51. In gewisser Weise spricht es also für die Korrektheit der Angabe im CIL, wenn ein Graffito nicht aufgefunden und folglich nicht überprüft werden konnte, da die Vergänglichkeit eines der Hauptmerkmale dieses Schreibmaterials ist. 
Tabelle 1: Schreibgeräte bei Graffiti in CIL IV (Gesamt = inklusive Pompeji, Herculaneum, Boscoreale, Boscotrecase, Neapel, Scafati, Stabiae).

\begin{tabular}{|c|c|c|c|c|}
\hline Schreibgerät & Spezifikation & Gesamt & Herculaneum & Pompeji \\
\hline \multicolumn{2}{|l|}{ carbo } & 244 & 27 & 207 \\
\hline \multirow[t]{4}{*}{ lapis } & ruber & 24 & 2 & 21 \\
\hline & gilvus/flavus & 7 & & 7 \\
\hline & albus & 6 & & 6 \\
\hline & unspezifiziert & 15 & & 15 \\
\hline \multirow[t]{4}{*}{ color } & ruber/rubrica & 19 & & 19 \\
\hline & niger & 11 & & 11 \\
\hline & albus & 1 & & 1 \\
\hline & flavus/gilvus & 1 & & 1 \\
\hline \multirow[t]{4}{*}{ gypsum } & rubrum & 5 & & 5 \\
\hline & gilvum/flavum & 2 & & 2 \\
\hline & album & 1 & & 1 \\
\hline & unspezifiziert & 6 & & 6 \\
\hline \multicolumn{2}{|l|}{ creta } & 5 & & 5 \\
\hline \multicolumn{2}{|c|}{ atramentum (Tinte) } & 4 & & 4 \\
\hline \multicolumn{2}{|l|}{ carbo vel lapis } & 2 & & 2 \\
\hline \multicolumn{2}{|l|}{ lapis vel creta } & 1 & & 1 \\
\hline \multicolumn{2}{|c|}{ color vel lapis ruber } & 1 & & 1 \\
\hline \multicolumn{2}{|l|}{ cuspis lignea } & 1 & & 1 \\
\hline \multicolumn{2}{|l|}{ ferramentum } & 1 & & 1 \\
\hline \multicolumn{2}{|l|}{ digitus } & 1 & & 1 \\
\hline \multicolumn{2}{|l|}{ terra fulva } & 1 & & 1 \\
\hline \multicolumn{2}{|c|}{ Gesamtzahl, inkl. geritzte Graffiti } & 6032 & 303 & 5211 \\
\hline \multicolumn{2}{|c|}{ Ohne geritzte Graffiti } & 359 & 29 & 319 \\
\hline \multicolumn{2}{|c|}{ Anteil der nicht geritzten Graffiti an der Gesamtzahl } & $5,95 \%$ & $9,57 \%$ & $6,12 \%$ \\
\hline \multicolumn{2}{|c|}{ Anteil der Kohlegraffiti an der Gesamtzahl } & $4,05 \%$ & $8,91 \%$ & $3,97 \%$ \\
\hline
\end{tabular}

Trotz dieser Vorbehalte wird eine Tendenz deutlich: Die Schreiber und Zeichner nutzten unterschiedliche Materialien und Werkzeugen. Der Anteil der Graffiti, die nicht eingeritzt wurden, wäre bei idealer Erhaltung vermutlich besonders in Pompeji nach oben zu korrigieren, wie der Vergleich mit Herculaneum nahelegt. Dennoch spricht der Umstand, dass in Kontexten, in denen geritzte und mit Kohle geschriebene Graffiti vorkommen, meist deutlich mehr geritzte Graffiti bekannt sind, dafür, dass dies bis zu einem gewissen Grad den antiken Begebenheiten entspricht. In der Casa del Gran 
Tabelle 2: Dokumentation von Kohlegraffiti in CIL IV Hauptband, Supplement 2 und Supplement 3.

\begin{tabular}{lrrr}
\hline CIL IV & Hauptband & Supplement 2 & Supplement 3 \\
\hline $\begin{array}{l}\text { Gesamtzahl der doku- } \\
\text { mentierten Graffiti }\end{array}$ & 1589 & 1937 & 2506 \\
\hline Davon Kohlegraffiti & 14 & 99 & 131 \\
\hline In Prozent & $0,88 \%$ & $5,11 \%$ & $5,23 \%$ \\
\hline $\begin{array}{l}\text { Kohlegraffiti in } \\
\text { Herculaneum }\end{array}$ & 0 von 3 & 0 von 3 & 27 von 297 = 9,09\% \\
\hline $\begin{array}{l}\text { Kohlegraffiti in } \\
\text { Pompeji }\end{array}$ & 14 von 1586 $=0,88 \%$ & 98 von 1886 = 5,19\% & 95 von 2042 = 4,65\% \\
\hline
\end{tabular}

Portale (V 35) in Herculaneum zum Beispiel sind im CIL vier textuelle Graffiti belegt (CIL IV 10589-10591. 10711). Hinzu kommen neun Zeichnungen von Gladiatorenhelmen (Langner 963-971) und eine weitere umfangreiche, noch unveröffentlichte Zeichnung. Von all diesen Graffiti ist nur einer in Kohle geschrieben (CIL IV 10589). Dieser befand sich jedoch in einem besonders ungeschützten Bereich, nämlich in einem begrünten kleinen Innenhof des Hauses. Heute ist er zwar nicht mehr zu erkennen, dennoch spricht dieser Befund dafür, dass sich Kohlegraffiti auch an einer solchen, der Witterung ausgesetzten Stelle bis zur Ausgrabung erhalten konnten. Die im Vergleich mit den anderen Bänden deutlich geringere Rate an Kohlegraffiti im Hauptband des CIL dürfte daher eher mit Verzögerungen bei der Dokumentation und dem Verlust der Wandoberflächen nach der Freilegung zusammenhängen. Insgesamt wurde aber tatsächlich sehr viel seltener mit diesem Material geschrieben und gezeichnet als mit einem spitzen Gegenstand. Die geritzten Graffiti machten sowohl in Pompeji als auch in Herculaneum die Mehrzahl aus. Die Kohlegraffiti dürften auch in den Regionen VI, VII und VIII in Pompeji, die im Hauptband des CIL behandelt werden, zwischen 5 und und $10 \%$ ausgemacht haben (Tabelle 2).

Für geritzte Graffiti und Zeichnungen konnte beinahe jeder spitze Gegenstand als Schreibgerät verwendet werden, sofern er härter war als der Stein, der Putz oder die Farbschicht, in die geschrieben werden sollte. Die Oberfläche musste zumindest so tief angekratzt werden, dass die Linie wahrzunehmen war. Langner zählt eine Reihe von Gegenständen auf, die als Schreibgeräte besonders in Frage kommen: Nägel, stili, Messer und fibulae waren besonders geeignet. Dabei ist es möglich, anhand des Aussehens des einzelnen Graffito in begrenztem Rahmen auf das verwendete Werkzeug zu schließen: Eine grobe Linienführung mit relativ flachen und schräg angeschnittenen Rändern deutet darauf hin, dass es sich bei dem Schreibgerät um eine flache Klinge handelte, wohingegen sehr feine Linien auf einen stark zugespitzten Gegenstand 
schließen lassen. ${ }^{367}$ Auch hier kann der heutige Erhaltungszustand des Putzes jedoch trügen oder eine genauere Beschreibung unmöglich machen, wenn die Oberfläche so stark abgerieben oder aufgeraut ist, dass die Umrisse der Linien schlecht definiert erscheinen. Um ein klareres Bild von den Schreibgeräten zu erhalten, ist es deshalb sinnvoll, auch Graffiti aus Innenräumen in Betracht zu ziehen.

In Herculaneum haben sich an den Wänden des schmalen Weges, der von der südwestlich oberhalb der Küste vorgelagerten Terrasse in die eigentliche Stadt führt, zahlreiche Graffiti erhalten, die nähere Aufschlüsse über die Schreibgeräte zulassen. Einer der sehr deutlich zu sehenden Graffiti an dieser Wand ist CIL IV 10703 (Abb. F37), der auf einer Höhe von ungefähr 1,40 m über dem Boden in die Wand geritzt wurde. Der Text beinhaltet nur den Namen Marcus und ist sehr leserlich geschrieben. Die Linien treten deutlich hervor, da sie vergleichsweise tief in den Putz eingeritzt und in gleichmäßiger, geschmeidiger Linienführung gezogen sind. Doch sie weisen bei relativ tiefem Profil nur eine Strichbreite von bis zu einem halben Millimeter auf, sodass nur ein sehr spitz zulaufendes Schreibgerät in Frage kommt. Varone bemerkt zu diesem Graffito, er sei „graphio scriptus“ - nimmt also an, dass ein Griffel oder stilus verwendet wurde. ${ }^{368}$ Dies ist überzeugend, gerade auch, weil für eine derartig ebenmäßige und weich gerundete Linienführung ein sehr hartes Schreibgerät nötig ist. ${ }^{369}$

Mit einem äußerst spitz zulaufenden Schreibgerät muss auch der Graffito CIL IV 10687, der sich ebenfalls an der Nordseite dieser schmalen Gasse befindet, geschrieben worden sein. Er ist im heutigen Befund nur schwer zu erkennen, doch die erhaltenen Buchstaben bilden eindeutig das Wort urso (Abb.F38). ${ }^{370}$ Die Linien sind

367 Vgl. Langner 2001, 13-14. Kritisch zu diesen Annahmen und mit experimentellen Methoden diese Annahmen hinterfragend: Lohmann 2018, 243-259.

368 Varone 2012, 514.

369 Es kann ausgeschlossen werden, dass der Putz noch weich war, als der Graffito geschrieben wurde, da sich keinerlei Aufwerfungen an den Linienrändern finden und stattdessen vielmehr kleine Abplatzungen zu beobachten sind. Ähnliche Befunde in Pompeji und Herculaneum: in Pompeji CIL IV 8048 aus der Casa dei Cei I 6, 15 (Varone 2012, 25); CIL IV 8173d in I 7, 18 (Varone 2012, 46; CIL IV 8216 in I 8, 17 (Varone 2012, 54); CIL IV 8392-8394 in der Casa degli Amanti I 10, 10-11 (Varone 2012, 80-81); einige Graffiti aus der Basilica VIII 1, 1-2 (Varone 2012, 348-395); CIL IV 5103 in IX 5, an der Außenwand im Bereich zwischen den Eingängen 16 und 17 (Varone 2012, 428); und in Herculaneum CIL IV 10529. 10534. 10537. 10538 IV 15-16, Raum 6, der neben dem Verkaufsraum liegt (Varone 2012, 487-488). Zur Verwendung von stili als Schreibgerät für Graffiti: Schaltenbrandt Obrecht 2012, 76-79. Schaltenbrandt Obrecht betont auch besonders die vielseitige Funktionalität der stili. Vgl. allgemein: Božic/Feugère 2004, 28-31; zum Magdalensberg: Öllerer 1998, 121-133; zu Funden aus Trier: Merten 1982, 17; zu Ostia: Drescher 1988, 285.

370 Varone hat diesen Graffito anscheinend mit CIL IV 10687 identifiziert, der urso lautet, aber laut CIL an der gegenüberliegenden Wand stand oder steht. Für CIL IV 10698 wird ein Fundort angegeben, der mit dem erhaltenen Graffito koinzidiert, doch dieser soll nach dem CIL das Wort zwei mal, also urso urso beinhalten, was so an der angegebenen Stelle nicht mehr zu erkennen ist. Daher ist es gut möglich, dass hier Ciprotti bei der Übernahme von Della Cortes Notizen ein Fehler unterlaufen ist, den Varone berichtigt hat. Vgl. Varone 2012, 511. 


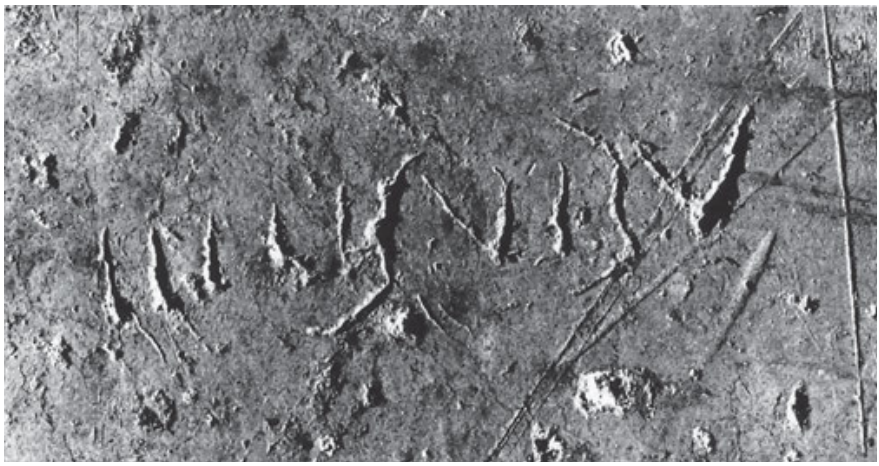

Abb. 24: CIL IV 10696 an der Nordseite des vicus sub insula IV in Herculaneum. (C) Su concessione del Ministero per i Beni e le Attività Culturali e per il Turismo - Parco archeologico di Ercolano. Jegliche weitere Reproduktion oder Duplikation ist untersagt.

haarfein und setzen an manchen Stellen der langen, geschwungenen Züge von $R$ und $S$ aus. ${ }^{371}$ Nur die oberste Schicht des geglätteten Putzes ist angekratzt. Abgesehen von den Sprüngen und kleinen Ausbrüchen beim V sind die Linien gleichmäßig tief und breit. Dies lässt vermuten, dass es sich bei dem Schreibgerät um einen sehr spitzen und dünnen Gegenstand handelte. Dafür käme entweder ebenfalls ein stilus oder aber eine Nadel, z. B. von einer fibula, in Frage. ${ }^{372}$

Ganz andere Charakteristika weist wiederum ein kurzer Graffito aus demselben Kontext auf. Bei CIL IV 10696 (Abb. 24) erscheinen die Linien breit und unregelmäßig. Manche sind sehr flach, andere sehr tief eingeritzt. Bei den ersten beiden Buchstaben ist der Schreiber offensichtlich abgerutscht und hat so je eine dünne Linie unterhalb des $I$ und des $P$ gezogen. Die drei $V$ sind untereinander sehr unterschiedlich und gerade beim ersten sowie bei dem darauf folgenden $S$ zeigt sich, dass er Mühe hatte, die Buchstaben zu formen. Doch wenn man bedenkt, dass der höchste Buchstabe, das $S$, nur $3 \mathrm{~cm}$ hoch ist, wird schnell deutlich, dass auch hier ein spitzes Schreibgerät verwendet wurde, allerdings mit sehr viel weniger Übung als Kraft.

Ein Beispiel für ein deutlich gröberes Schreibwerkzeug findet sich in diesem Kontext nicht, dafür aber an Grab EN 6 in der Nekropole vor der Porta di Nocera (Abb. F39). Der Graffito (CIL IV 10222 = Langner 294) zeigt einen Kopf im Profil und den in den Kopf eingeschriebenen Text Promus fel $<$ l $>$ ator.${ }^{373}$ Die Zeichnung ist ca. $7 \mathrm{~cm}$ hoch und die Linien sind mindestens $1 \mathrm{~mm}$ breit. Die Linie, die den oberen Rand des Schädels abbildet, erscheint besonders flach, aber breit und an der Ränder ausgerissen. Dies deutet darauf hin, dass der Zeichner und Schreiber hier ein grobes Utensil verwendete,

371 Auf Varones Foto erscheinen die Striche dicker als sie es tatsächlich sind, da er auch hier mit Streiflicht gearbeitet hat, um die Sichtbarkeit zu erhöhen. Der Unterschied in der Wirkung der Fotografien zeigt sich deutlich z. B. an der abgeplatzten Stelle zwischen $R$ und $S$.

372 Auch ein oskischer Graffito im Vestibül der Casa Sannitica (V 1) in Herculaneum muss mit einem extrem feinen Schreibgerät geschrieben worden sein. Die Buchstaben sind ca. $1 \mathrm{~cm}$ hoch und scheinen jeweils die eigentlichen Buchstabenformen mit sehr dünnen Linien zu umranden: vgl. Antonini 2007, 89. 87 Taf.4.5.

373 Vgl. Langner 2001, 13; 14 Abb. 1f; Taf. 14. 


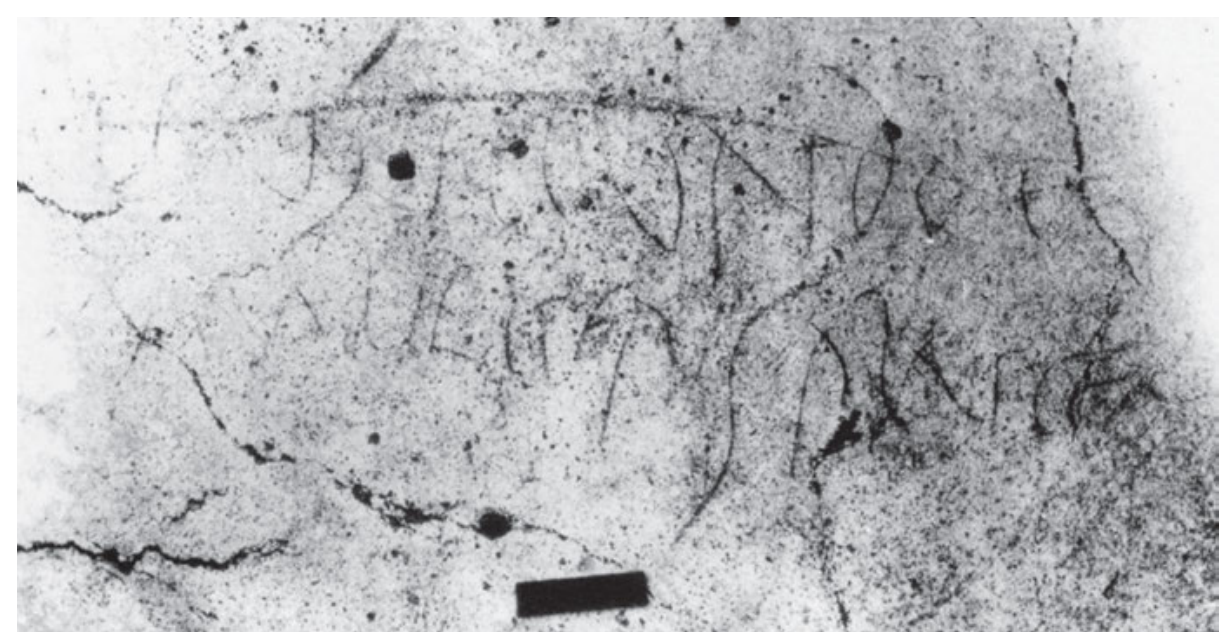

Abb. 25: Kohlegraffito CIL IV 10247 an der Fassade von Grab ES 11 in der Nekropole an der Porta Nocera. ( ) Su concessione del Ministero per i Beni e le Attività Culturali e per il Turismo - Parco archeologico di Pompei. Jegliche weitere Reproduktion oder Duplikation ist untersagt.

das er teilweise fest aufsetzte, teilweise aber auch mit weniger Druck über die Oberfläche führte, wie unterhalb des Kinnes und am Hals. ${ }^{374}$

Einen Sonderfall stellen die in den noch feuchten und daher weichen Putz geschriebenen Graffiti aus dem Frauentrakt der Therme in Herculaneum sowie aus dem lupanar, den Stabianer Thermen und an der Fassade des Hauses II 1, 10 in Pompeji dar, die bereits im Zusammenhang mit den Beschreibflächen besprochen wurden. ${ }^{375}$ Hier kommen auch weniger spitz zulaufende und weniger stabile Gegenstände als Schreibwerkzeuge in Betracht. Auch ein Stöckchen oder etwa eine Haarnadel aus Bein wäre stabil genug, um den Putz einzudrücken (Abb. F35. F36). ${ }^{376}$

Die größte Gruppe unter den Graffiti, die nicht eingeritzt, sondern aufgetragen sind, sind die mit Kohle geschriebenen und gezeichneten Graffiti. $\mathrm{Zu}$ den 207 textuellen Kohlegraffiti in Pompeji kommen 24 Zeichnungen, die im CIL erwähnt werden. ${ }^{377}$ Aus Herculaneum sind 27 Texte und eine Zeichnung bekannt (vgl. Tabelle 1, S.93, und Tabelle 2, S.94). Der Großteil dieser Zeugnisse ist heute nicht mehr erhalten. ${ }^{378}$ Auf einem Foto von CIL IV 10247 aus dem Jahr 1957 (Abb.25), das kurz

374 Langner 2001, 13; Varone 2012, 461: litteris modice crassis penitus scariphatis.

375 Vgl. Kap. 2.2.3.1, S.90.

376 Auch für die Vorbereitung von Wandmalereien wurden vermutlich hölzerne Zeichengeräte verwendet: Allag/Barbet 1972, 985.

377 Langner 2001, 12-13 Anm. 8.

378 Vgl. dazu Benefiel 2011, 39. Fotos finden sich in Varone 2012 von den Kohlegraffiti CIL IV 6897. 8232. 10231. 10232a. 10232b. 10233. 10234. 10247. 10500. apographa sind im CIL und/oder bei Varone bei den Nummern CIL IV 3932. 8222. 8399. 8404. 8883. 8910. 8931. 9100. 9155. 9189 abgedruckt. 

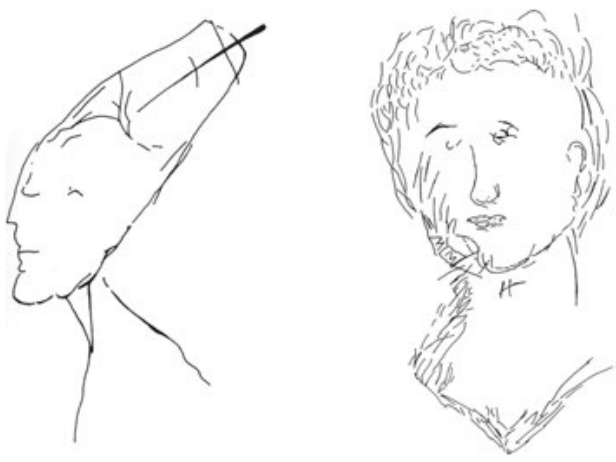

Abb. 26: Kohlezeichnung eines menschlichen Kopfes an Grab EN 12 in der Nekropole an der Porta Nocera, links Langner 306, rechts Langner 570.

nach der Ausgrabung aufgenommen wurde, ist der Graffito noch sehr deutlich zu erkennen. Er befand sich an der Fassade von Grab ES 11 in der Nekropole an der Porta di Nocera. Inhaltlich ist er nicht auffällig: Habitus Secundo et suis / amabiliter salutem. An der Gestaltung jedoch fällt auf, dass die einzelnen Linien etwas weicher gerundet sind. Die meisten Buchstaben sind in Kursiven ausgeführt, doch die $<$ E $>$ s oder auch das $<\mathrm{L}>$ in amabiliter sind an den monumentalis-Formen orientiert. Die Linienführung ist anders als bei den geritzten Graffiti: Besonders beim $\langle\mathrm{S}\rangle$ von salutem ist gut $\mathrm{zu}$ erkennen, dass das Kohlestückchen etwas abgeflacht war, wodurch sich die Breite der Linien ändert. Dies wie auch die Rundungen sind nur möglich, weil der Schreiber deutlich weniger Kraft aufwenden musste. Auch bei schlichteren Zeichnungen, die sich in Grab EN 12, ebenfalls in der Nekropole an der Porta di Nocera erhalten haben (Langner 306 und 570) (Abb.26), fällt auf, dass der Zeichenstil durch kurze, immer wieder abgesetzte Linien geprägt ist. Nicht nur die Haare, sondern auch die Umrisse des Halses werden auf diese Weise angegeben. Eine weitere, ebenfalls noch recht gut erhaltene Zeichnung ist ein menschlicher Kopf, der ebenfalls an Grab EN 12 in der Nekropole vor der Porta Nocera gezeichnet wurde (Abb. F40).

Die verschiedenen Stein-, Gips- und Kreidesorten, die im CIL erwähnt werden, bilden zusammen eine weitere Gruppe unter den Schreibmaterialien. Während in Herculaneum lediglich zwei Graffiti (CIL IV 10704 und 10705) lapide rubro geschrieben sind, beläuft sich die Zahl der in rot, schwarz, gelb oder weiß aufgebrachten Graffiti in Pompeji auf immerhin $68 .^{379}$ Die Zeichnung Langner 461 ist mit einer rötlichen Farbe auf den hellen Putz des Grabes EN 12 gezeichnet (Abb. F40). Dargestellt ist ein menschlicher Kopf. Die einzelnen Linien sind recht breit und die Zeichnung wirkt grob und unbeholfen. Das Zeichengerät war sicherlich kein Stift, sondern eher ein Stein oder eine rote Scherbe. Die beiden Graffiti CIL IV 8426 und $8436^{380}$ erscheinen dagegen flüssiger und feiner, weisen aber in der Linienführung auch mehr Redundanzen auf.

379 Davon sind nur zu zweien Fotos bei Varone vorhanden: CIL IV 8426 und 8436 . Zu weiteren neun sind aber bei Varone, im CIL und bei Langner Umzeichnungen zu finden: CIL IV 8003, 8227, 8309, 9077a, 9172 und 9173 sowie Langner 461, 774 und 806.

380 Abbildungen: Varone 2012, 85. 
Dieses heterogene Bild wird ergänzt durch die apographa von CIL IV 8003 und 9077a bei Varone ${ }^{381}$ sowie 8227, 8309, 9172 und 9173 im CIL.

\subsubsection{Schriftarten und Gestaltung}

Die Buchstabenformen sind ein wichtiges gestalterisches Charakteristikum der Graffiti. Denn anders als Steininschriften und Dipinti sind die meisten Graffiti in Kursiven geschrieben. Das Aussehen der Kursivschrift steht, wie Armando Petrucci betont, in direktem Zusammenhang mit den antiken Schreibpraktiken des Graffitischreibens, des Schreibens auf Papyrus und in Wachs. Besonders markante Buchstaben sind das $\langle\mathrm{A}\rangle$, das $\langle\mathrm{O}\rangle$, das $\langle\mathrm{E}\rangle$, das $\langle\mathrm{F}\rangle$ und das $\langle\mathrm{R}\rangle$ (Abb. F41). Bei diesen, aber auch im Allgemeinen, ist zu beobachten, dass Rundungen und Richtungswechsel vermieden wurden und viele vormals und in anderen Schriften schräge oder waagerechte Linien zu senkrechten Strichen umgewandelt wurden. Der Querbalken des $<\mathrm{A}>$ wird dann senkrecht unterhalb des Scheitels positioniert, das $<\mathrm{O}>$ besteht aus zwei, oft nur leicht gebogenen Linien und ist unten offen, $<\mathrm{E}>$ und $<\mathrm{F}>$ werden aus zwei parallelen senkrechten Linien gebildet und das $<\mathrm{R}>$ öffnet sich ebenfalls. Dies hängt eng mit der erwünschten Schreibgeschwindigkeit bei der Anfertigung von geschäftlichen Schriftstücken aber auch dem mühsamen Einritzen von kleinteiligen Buchstaben in so harte Oberflächen wie Putz oder gebrannten Ton zusammen. Die Gestaltung der Buchstaben $\langle$ B $>$ und $\langle$ D $>$ hingegen sieht Petrucci sicherlich zurecht durch das Schreiben auf weicherem Untergrund, also Wachstafeln oder Papyrus motiviert. Hier wird zwar die Anzahl der Züge reduziert, doch die jeweils zwei verbleibenden sind stark gekrümmt. Zudem fügen sich die Buchstaben der kursiven capitalis nicht in ein Zwei-, sondern in ein Vierlinienschema, da die Ober- und Unterlängen einiger Buchstaben weit über die Grundlinie und die H-Linie hinausragen. ${ }^{382}$

Ein deutliches Beispiel dieser Schrift stellt der Graffito CIL IV 5380 dar, der eine Liste von verschiedenen Lebensmitteln mit Datums- und Zahlenangaben umfasst (Abb. 27). Die Kursive war die Alltagsschrift all derer, die häufig kleinformatige Texte schrieben oder lasen. Insbesondere der stilus ist daher als Schreibgerät mit dieser Schrift korreliert, da Personen, die häufig einen solchen bei sich hatten, es gewohnt waren, diese Schrift zu verwenden. Sie ist grundsätzlich nicht weniger konventionalisiert oder schlichter als die monumentalis oder die actuaria, sondern verweist lediglich in ein anderes Praxisfeld der Schrift.

Unter den Graffiti sind auch zahlreiche Beispiele für stark an diese Monumentalformen angelehnte Schriften, welche für großformatige Dipinti und Steininschriften

381 Varone 2012, 17 und 321.

382 Dazu besonders übersichtlich: Petrucci 1992, 45-47. Robert Marichal sieht dagegen keine kausale Verbindung zwischen dem harten Schreibuntergrund und den Buchstabenformen: Marichal 1967, 152 mit Bezug auf Solin et al. 1966, 51, wo Solin durch eben diese Verbindung die offenen Formen von $<0>$ und $<$ V $>$ erklärt. 


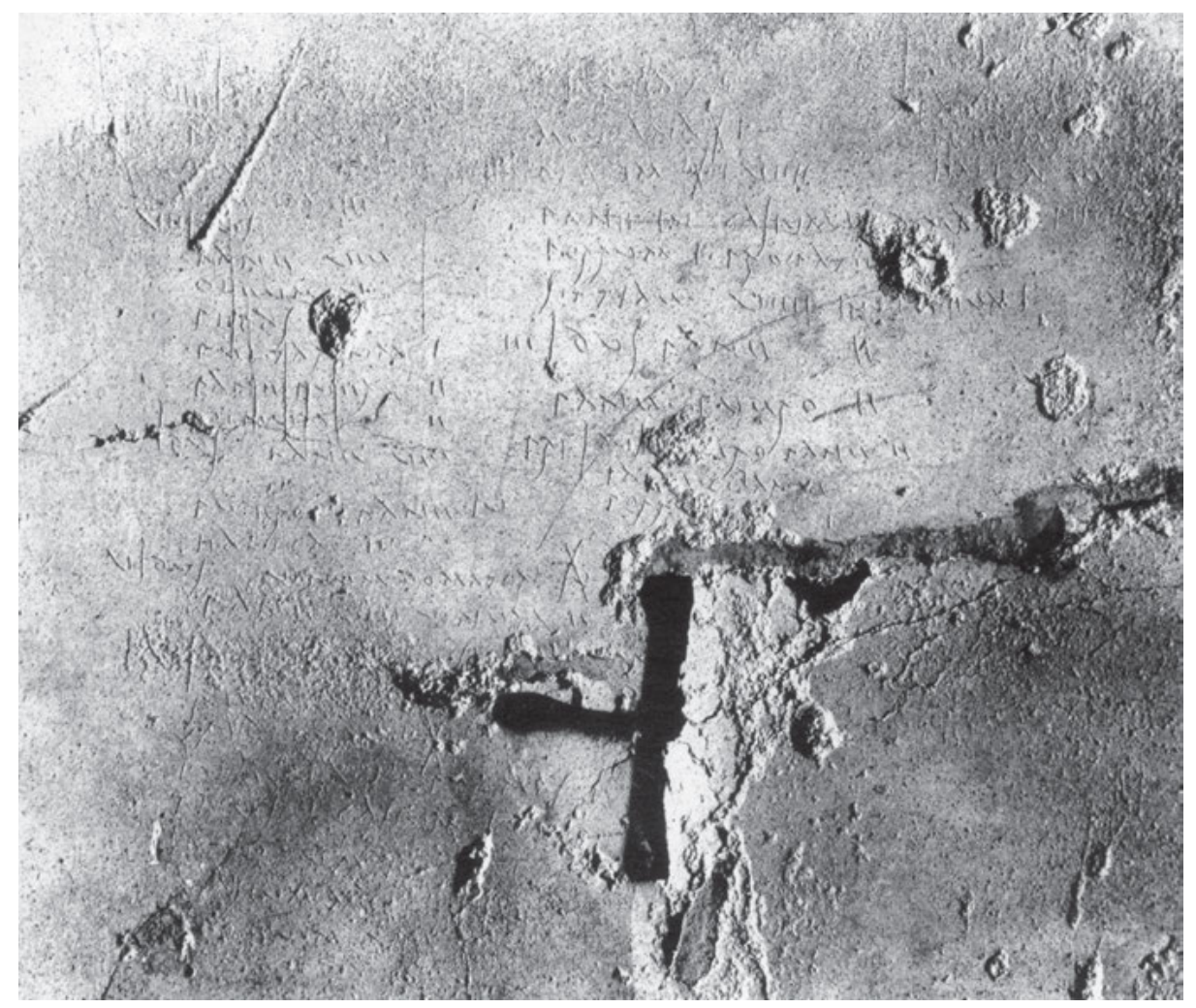

Abb. 27: CIL IV 5380 aus Raum t in IX 7, 25. (C) Su concessione del Ministero per i Beni e le Attività Culturali e per il Turismo - Parco archeologico di Pompei. Jegliche weitere Reproduktion oder Duplikation ist untersagt.

gebräuchlich waren. Dazu zählt ein Graffito aus der Casa dei Quattro Stili (I 8, 17) (CIL IV 8215). Der Text lautet: quos L V P amat valeant und ist in eleganter und deutlich an formalen Idealen öffentlich sichtbarer Inschriften orientierten Buchstaben geschrieben. ${ }^{383}$ Ähnlich verhält es sich auch bei dem bereits erwähnten Graffito CIL IV 9127. Hier sind die Buchstaben - vermutlich auch mit Bezug auf die umstehenden Dipinti - in den gelängten Formen der actuaria gestaltet. Bei einigen Graffiti, die in ihrer Formensprache im Allgemeinen eher der Kursiven entsprechen, finden sich Mischformen aus dieser und anderen Buchstabenformen.

Besonders oft kommt es vor, dass die beiden Formen des $\langle\mathrm{E}\rangle$, also $\langle\mathrm{E}\rangle$ und $\langle\|\rangle$ ausgetauscht werden und gelegentlich auch beide innerhalb derselben Inschrift vorkommen. Bei CIL IV 8161 kommt zum Beispiel ein kursives $<R>$ direkt neben einem

383 Der Graffito wird ausführlich auch von Benefiel besprochen: Benefiel 2011, 35. 33 Abb. 2.3. Abbildung: Varone 2012, 54. 
nicht kursiven <E $>$ vor. Daran wird deutlich, dass zumindest für Graffiti die Beschreibung der Schriftart „Kursive“ nicht als streng befolgtes Schema betrachtet werden kann, sondern dass die Schreiber sich relativ frei je nach Kenntnis und Können die bevorzugten Buchstabenformen aus verschiedenen Registern zusammenstellten. Dass gerade in längeren Graffiti, wie der Liste oder der Ansammlung von Epigrammen (CIL IV 4966-4972) ${ }^{384}$ von der Außenwand des odeion in Pompeji (VIII 7, 19) oder auch dem Epigramm CIL IV 10697 aus Herculaneum dennoch vor allem Kursive verwendet wurden, ist der technischen Schwierigkeit und Mühsal geschuldet, die das Schreiben in harten Putz bedeutete. Dies ist zum Beispiel bei einigen Graffiti, die ebenfalls in der Casa dei Quattro Stili zu lesen sind, festzustellen: CIL IV 8218a-1. 8219a-c. Wie Benefiel bemerkt, legen die Formen der Buchstaben nahe, dass die Schreiberinnen, die namentlich genannt werden, nicht allzu erfahren waren. ${ }^{385}$

Eine weitere Variable stellt die Höhe oder Größe der Buchstaben dar, die sowohl im Zusammenhang mit dem Vorgang des Schreibens als auch für Fragen nach der Sichtbarkeit und dem Verhältnis zu umliegenden Inschriften von Bedeutung ist. Die kleinsten Buchstaben sind nur wenige Millimeter hoch, wie etwa CIL IV $10694 \mathrm{mit}$ $2 \mathrm{~mm}$ oder CIL IV 10697 mit $3 \mathrm{~mm}$. Demgegenüber stehen kolossal wirkende Exemplare mit über $20 \mathrm{~cm}$ Buchstabenhöhe, wie CIL IV 10564 in den fauces der Casa Sannitica (V 1) in Herculaneum. Bei den meisten Graffiti bewegt sich die Höhe der Buchstaben allerdings zwischen ca. $1 \mathrm{~cm}$ und ca. $10 \mathrm{~cm}$. Dabei können die Ober- und Unterlängen einzelner Buchstaben weit ausgreifen, wie zum Beispiel beim $C$ und bei $D$ von cecidisse in CIL IV 1904 (Abb. F49).

Auch bei den Graffiti nutzten die Schreiber unterschiedliche Formen des Layouts oder der Text-/Bild-Anordnung. Dies lässt sich nicht bei allen Untergruppen gleichermaßen feststellen, trifft aber besonders auf längere Graffiti zu. Mehrzeilige Graffiti können zum Beispiel längere Listen wie die bereits erwähnte Lebensmittelliste CIL IV 5380 (Abb.27) oder auch die Auflistung von Gerätschaften und Einrichtungsgegenständen CIL IV 10566 aus dem nymphaeum der Casa di Nettuno e Anfitrite (V 6-7) in Herculaneum sein. ${ }^{386}$ Auch Versinschriften stellen in dieser Hinsicht eine interessante Untergruppe des Materials dar, das von Kruschwitz ausführlich untersucht wurde. Er kann am Beispiel der Graffiti aus der basilica zeigen, dass bei Versinschriften meist eine Korrelation zwischen Vers und Zeile festzustellen ist. ${ }^{387} \mathrm{Nicht}$ immer jedoch koinzidieren Vers- und Zeilenende, da auch der Inhalt der Texte und

384 Vgl. Kap. 3.2.2, S. 139. Zu diesen Epigrammen siehe auch: Morelli 2000, 237-257. 104-107 Abb. 6-9; Alfredo Morelli betont, dass sie alle von derselben Hand stammen: 239.

385 Besonders bei 8218a, vgl Varone 2012, 52. Benefiel 2011, 40-41.

386 Vgl. das apographon im CIL-Eintrag.

387 Kruschwitz betont hier zudem generell die Notwendigkeit, solche Layouts auch für Graffiti zu untersuchen: „How are verse inscriptions presented on the walls of Pompeii?“ Dahinter steht die Frage, wie durch visuelle Mittel die Wahrnehmung von Texten gesteuert wird: Kruschwitz 2008, 226-228. 231. 237. 


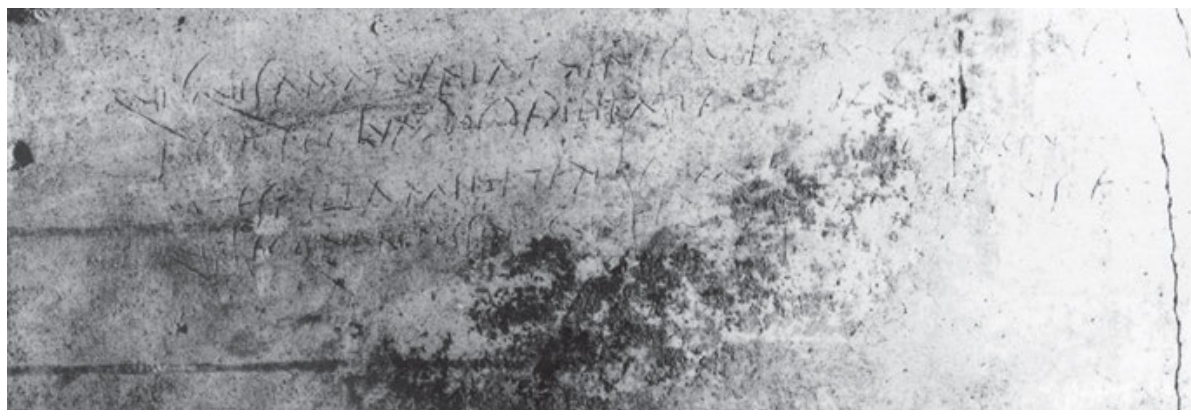

Abb. 28: CIL IV 1824. (C) Su concessione del Ministero per i Beni e le Attività Culturali e per il Turismo Parco archeologico di Pompei. Jegliche weitere Reproduktion oder Duplikation ist untersagt.

äußere Gegebenheiten die Schreiber dazu bringen konnten, weniger oder mehr Wörter in einer Zeile unterzubringen. ${ }^{388}$ Dennoch kann an den Graffiti nachvollzogen werden, dass die Schreiber sich oft bemühten, sowohl die einzelnen Verse als auch das Metrum als gliedernde Elemente in den Wandinschriften umzusetzen, da sie oft nicht nur genau einen Vers in eine Zeile schrieben, sondern bei im elegischen Distichon verfassten Gedichten auch zwischen Hexameter und Pentameter unterschieden, indem sie den Pentameter leicht einrückten, wie zum Beispiel bei CIL IV 1824 (Abb.28). ${ }^{389}$

Mit Bezug auf die Kombination von Text und Bild fallen unter den Graffiti aus Pompeji und Herculaneum besonders zwei Phänomene auf. Zum einen zählt dazu die Rahmung von Texten, besonders in Form von tabulae ansatae, aber auch anderen ikonographisch schwächer spezifizierten Formen. Und zum anderen spielen, besonders unter den pompejanischen Graffiti Text/Bild-Kombinationen eine große Rolle. Eine Sonderrolle nehmen dabei die sogenannten Namensschiffe ein, die sowohl in Pompeji als auch in Herculaneum zahlreich belegt sind. Dabei werden meist ein oder zwei Buchstaben so geformt, dass sich eine Bootsdarstellung ergibt. Nahezu unerlässlich ist das $\langle$ S $\rangle$, welches weit zurückschwingend den Schiffsrumpf bildet. Oft tritt ein Segel, das aus einem $\langle\mathrm{T}\rangle$ gebildet wird, hinzu und in einzelnen Fällen wird die Darstellung detaillierter ausgearbeitet, indem weitere Linien zu Rudern verlängert werden, wie zum Beispiel bei CIL IV 4755 (Abb. 29). ${ }^{390}$

388 Dies ist zum Beispiel bei CIL IV 1227 der Fall, welches in die Kannelur einer Säule eingeschrieben ist, betrifft aber auch Unebenheiten oder bereits bestehende Inschriften im Kontext. Kruschwitz 2008, 236. 248-249. Bei CIL IV 1904 wurden aus nicht voll nachvollziehbaren Gründen die Worte qui tot noch in die erste Zeile gezogen. Darauf geht zum Beispiel Gigante nicht ein, wenn er CIL IV 1904 in zwei Zeilen nach Versen getrennt abdruckt: Gigante 1979, 233. Vgl. dazu Kruschwitz 2008, 240-241.

389 Kruschwitz 2008, 238-239, vgl. auch CIL IV 1860. Abbildungen zu den beiden Graffiti: Varone 2012, 352. 368.

390 Für weitere Buchstabenschiffe s. Langner 1-27. Langner sieht die Präferenz von Schiffen für Buchstaben- und insbesondere Namensbilder zum einen darin begründet, dass sich die lateinische Kursivschrift besonders gut dafür eignete (Vgl. CIL IV 10036) und zum anderen darin, dass das Boot 


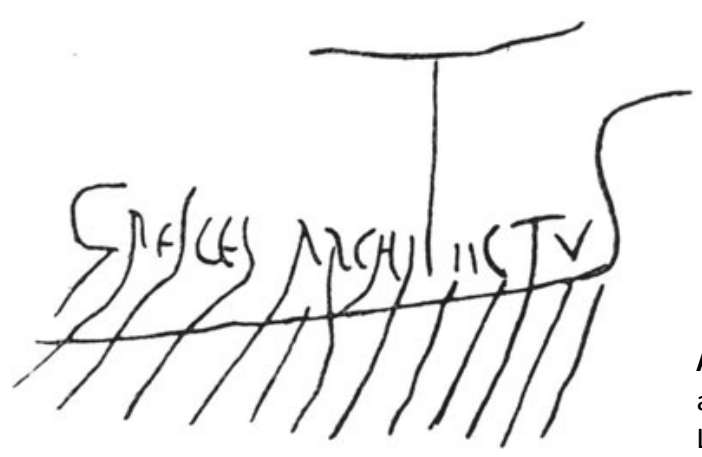

Abb. 29: Namensschiff Cresces architectus aus dem Peristyl von VII 7, 5: CIL IV 4755/ Langner 8.

Eine häufige Art der Rahmung von Graffiti war die tabula ansata. Campbell und Kruschwitz untersuchen in einem Aufsatz von 2009, wie Texte in Graffiti und Dipinti dargestellt wurden und zeigen, dass vor allem Namen, aber auch Grüße, Aufforderungen und Wünsche auf diese Weise als monumentalisierte Schrift ausgezeichnet werden konnten. ${ }^{391}$ Gerade bei den Grüßen erscheint es jedoch fraglich, ob tatsächlich der Wunsch nach Monumentalisierung im Vordergrund stand und ob die Rahmung den eingeschriebenen Text in jedem Fall zu einem dargestellten Text macht. Eine einfachere Erklärung, die auch von den Rezipienten weniger Transferleistung erfordert hätte, wäre, dass die Rahmung und insbesondere die mit einer tabula ansata den Text aus dem Putzuntergrund und aus der Masse der Graffiti hervorhob und ihn leichter auffindbar machte. ${ }^{392}$ Auch Langner misst den tabulae einen bedeutenden Bildwert bei und bietet zudem weitere Aspekte als Erklärungen an. So sieht er diese Form der Rahmung auch als Möglichkeit, Beischriften als Titel, Motto oder Bildunterschrift auszuzeichnen. ${ }^{393}$ Dies ist letztendlich nur für den einzelnen Kontext zu entscheiden.

In Pompeji und Herculaneum kommen auch Text-Bild-Kombinationen vor, in denen sich die beiden Komponenten sowohl inhaltlich als auch formal ergänzen. Besonders häufig sind Namen und Grüße sowie Diffamierungen in Kombination mit phalli ${ }^{394}$ oder auch mit Porträts und Karikaturen anzutreffen. ${ }^{395}$ Beide Kombinationen sind ambivalent und können durch verbale und zeichnerische Details wie sexuelle Beleidigungen oder Ausdrücke von Bewunderung wohlwollend oder spottend gemeint sein. Kopf- und Gesichtsdarstellungen bieten großen Spielraum für Karikaturen, die Entsprechungen im textuellen Bestandteil des Graffito finden oder auch nur durch

auch inhaltlich als Überbringer einer Nachricht gedeutet werden konnte: Langner 2001, 28. Zu dieser Gattung liegt ein ausführlicher Beitrag von Maiuri vor: Maiuri 1958b. Siehe auch: Pekáry 1999, 420 und Lohmann 2018, 266-270.

391 Kruschwitz/Campbell 2009, 59-66. 70; tabulae ansatae spielten ansonsten auch eine große Rolle als Votivgaben: Meyer 2004, 28 Anm. 38.

392 Vgl. Kap. 2.2.2.4, S. $83 f$.

393 Langner 2001, 27.

394 Langner 2001, 32.

395 Langner 2001, 34-42. 


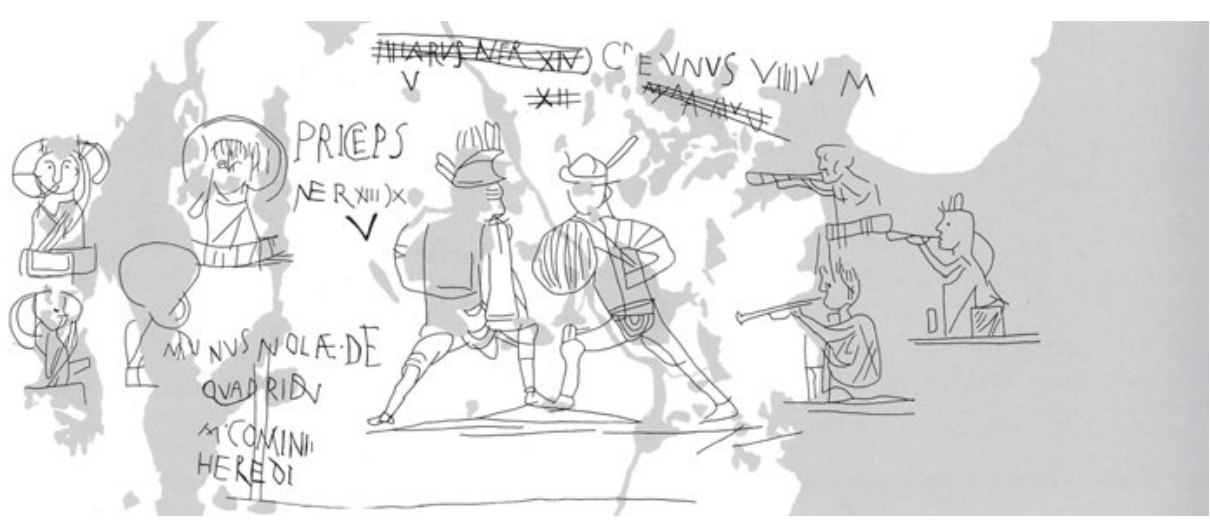

Abb. 30: Gladiatorenkampfszene von Grab EN 12 in der Nekropole an der Porta Nocera, CIL IV 10237/ Langner 1007.

eine Namensbeischrift auf eine bestimmte Person abzielen können. ${ }^{396}$ Interessant in Bezug auf Text/Bild-Kombinationen sind auch einige Gladiatorenbilder. Besonders detailreich ist zum Beispiel Langner 1007 mit den Inschriften CIL IV 10237 (Abb. 30. F44).

Einige Graffiti sind nicht nur in einer gegenüber der Kursiven formelleren Form der capitalis monumentalis geschrieben, wie etwa CIL IV 5325 (Abb.F32) oder die bereits genannte Inschrift CIL IV 9127, sondern stellen durch ihre Formen wichtige Verbindungen zwischen den verschiedenen Schreibtechniken der Steininschriften, Dipinti und Graffiti her. Die Graffiti CIL IV 1385a, 4119, 8388c, 8388d, 8631, 8893, 10203b und ein isoliertes „B“, das zwar nicht im CIL veröffentlicht, aber auf einem Foto bei Varone zu finden ist, weisen doppelt gezogene Linien und deutlich als capitalis monumentalis ausgeführte Buchstabenformen auf. ${ }^{397}$ Interessant ist dabei nicht nur, dass die Schreiber hier mit der Variabilität und den verschiedenen verfügbaren Registern spielten, sondern dass sie offensichtlich auch die Formen und deren Konstruktion reflektierten.

$396 \mathrm{Zu}$ verschiedenen Arten der Karikatur und zu der Notwendigkeit, dem Spott eine Richtung zu verleihen: Langner 2001, 34.

397 Abbildungen: CIL IV 1385a: Varone 2012, 262; CIL IV 4119: Varone 2012, 214; CIL IV 8388c und 8388d: Varone 2012, 80; CIL IV 8631: Varone 2012, 148; CIL IV 8893: Varone 2012, 207; CIL IV 10203b: Varone 2012, 283; B oberhalb von CIL IV 1854d: Varone 2012, 364. Hinzuzählen könnte man auch CIL IV 8129 (Varone 2012, 39), welches aber stark gelängte Formen und nur teilweise doppelte Linien aufweist. 


\subsubsection{Zwischenergebnis}

Dipinti und Graffiti existierten in Pompeji in direkter Nachbarschaft, da sie grundsätzlich auf die gleichen Beschreibflächen geschrieben wurden: den Wandputz der Fassaden und Wände. Dabei treten aber nicht nur Abweichungen auf, die daher rühren, dass Dipinti stärker auf öffentliche Räume und stark frequentierte Orte hin ausgerichtet sind, sondern auch innerhalb der einzelnen Kontexte wurde der untere Fassadenbereich mit Graffiti beschrieben, während die Dipinti sich in der oberen Zone konzentrierten. Dies lässt sich bei näherer Betrachtung primär durch materialitätsbedingte Faktoren wie die Beschaffenheit des Putzes erklären. Auch die Sichtbarkeit der jeweiligen Stelle spielte eine große Rolle. Es kam den Schreibern der Graffiti möglicherweise sehr zupass, dass sie beim Schreiben in der unteren Zone den Vorgang mit ihrem Körper optisch vor dem Blick von Passanten abschirmen konnten.

Das Spektrum und die Spezifikationen der Schreibgeräte helfen bei den Graffiti, sie in den Zusammenhang bestimmter Praktiken einzuordnen. Die Bandbreite reicht dabei von professionellen Schreibgeräten über zweckentfremdete Utensilien bis hin zu Gegenständen, die kaum einer spezifischen Funktion zugeordnet werden können. Zuerst ist dabei an die stili zu denken, die von Personen, die regelmäßig mit der Tätigkeit des Schreibens beschäftigt waren, mitgeführt wurden. ${ }^{398}$ Doch auch Schlüssel und andere mehr oder weniger spitze Metallgerätschaften sind aus den Befunden abzuleiten. Und schließlich ist etwa bei Steinen oder Scherben auch die Möglichkeit zu bedenken, dass der Schreiber oder Zeichner diese gar nicht mitgebracht hatte, sondern sie vorfand und sich spontan dazu entschloss, einen Graffito anzufertigen. Diese Werkzeuge stehen in engem Zusammenhang mit der Gestaltung der Texte und Bilder - nicht nur aufgrund ihrer physischen Eigenschaften, sondern auch, weil ein professionelles Schreibgerät in der Hand eines geübten Schreibers zu einem ganz anderen Erscheinungsbild führt als etwa eine Scherbe in der Hand einer Person, die nur selten schreibt.

Bei den Graffiti unterscheiden sich die Buchstabenformen optisch meist deutlich von denen der Dipinti und der in Stein gehauenen Inschriften. ${ }^{399}$ Als Schriftarten kommen vor allem kursive Buchstabenformen vor, aber auch solche, die an die capitalis monumentalis angelehnt sind, und vereinzelt actuaria vor. Das Format der Graffiti ist meistens um ein Vielfaches kleiner als das der Dipinti und der Steininschriften. Auch die Anordnung der einzelnen Buchstaben untereinander und die Variation von Buchstabenformen erfolgten nach anderen Mustern als bei Dipinti und Steininschriften, bei denen die Sichtbarkeit und Leserlichkeit eine große Rolle spielten. Sowohl der Schreibprozess als auch die Wahrnehmung dieser Bilder und Texte müssen daher anders verlaufen sein.

398 In eine ähnliche Kategorie würden Hammer und Meißel fallen, die jedoch in Pompeji nicht als Graffiti-Werkzeuge belegt sind. Vgl. Chaniotis 2011, 193.

399 Solin et al. 1966, 50-51. Marichal 1967, 151-154. 


\subsubsection{Zwischenergebnis zur Materialität und Gestaltung der Schriftzeugnisse in Pompeji und Herculaneum}

Die zu Beginn dieses Kapitels gestellten Fragen zielten auf vier übergreifende Aspekte ab: 1. Die Konventionen bei der Gestaltung verschiedener Inschriften und deren Korrelation mit einzelnen Inhalten, 2. Techniken und Werkzeuge, 3. Die Personenkreise, die Inschriften schrieben, und 4. Hinweise auf die Rezeption der verschiedenen Inschriften.

Mit Blick auf die Beziehung von Gestaltung und Inhalt konnten wichtige Merkmale festgestellt werden, die darauf hindeuten, dass von Seiten der Betrachter und Leser ein Teil der Interpretationsleistung über die Form und das Erkennen konventionalisierter Gestaltungsmuster geleistet wurde. Das gilt vor allem bei Steininschriften und bei Dipinti, wo das inhaltliche Spektrum vergleichsweise eng umrissen ist. Hier scheinen Gestaltungsmuster etabliert gewesen zu sein, auch wenn es Abweichungen und Ausnahmen gibt. Relevante Merkmale im formalen Bereich sind hierbei die Schriftart, die Textverteilung, die Größe der Inschrift, die Materialien und die Wahl der Anbringungsstelle. Diese gelten für die verschiedenen inhaltlichen Typen in jeweils unterschiedlicher Ausprägung.

Besonders bei den programmata ist dabei zu fragen, was man sich davon erhoffte, wenn die Inschriften einem etablierten Schema entsprachen. Sie waren so zwar als Wahlwerbungen zu erkennen, doch gerade an dicht beschriebenen Flächen konnten völlig konventionell gestaltete derartige Inschriften doch wohl kaum die erwünschte Aufmerksamkeit für den einzelnen Bewerber erregen. Allerdings waren die programmata mit Sicherheit nicht das einzige Mittel der Bewerbung eines Kandidaten um ein Amt. Vielmehr ermöglichten persönliche Beziehungen, die zwar in den

Dipinti Widerhall finden können, aber nicht unbedingt müssen, den Erfolg. ${ }^{400}$ Daher muss es nicht zwangsläufig das Ziel gewesen sein, größtmögliche Aufmerksamkeit zu erzielen. Welche anderen Gründe sind plausibel dafür, dass die Namen und Eigenschaften der Kandidaten in immer wiederkehrenden, standardisierten Formulierungen und Floskeln in großer Zahl im ganzen Stadtgebiet als Inschriften angebracht wurden und dass man dafür professionelle Schreiber engagierte ${ }^{401}$ Eine Stärke der programmata war, dass man die entsprechenden Aufträge mit einem Minimum an Planung vergeben konnte. Den Schreibern waren im Minimalfall nur der Name und das angestrebte Amt mitzuteilen, den Rest der ,Angaben', wie vir bonus oder dignus rei publicae konnten sie ergänzen. Ähnlich muss es auch denjenigen, die die Empfehlungen tatsächlich lesend rezipierten, ergangen sein. Wenn ein Kandidat als vir bonus bezeichnet wurde, sagte das kaum etwas über seine persönlichen Qualitäten aus. Diese mussten dem Leser bereits bekannt sein oder etwa durch Veranstaltungen und gegebenenfalls nur deren Ankündigung bekannt gemacht werden.

400 Vgl. dazu Mouritsen 1988, 44-69 besonders 64.

$401 \mathrm{Zu}$ diesem Aspekt, siehe ausführlich: Kapitel 5.1.2. 
Offenbar wurde der Gestaltung ein hoher Wert beigemessen. Allerdings ging es augenscheinlich weniger um Individualität oder das Wiedererkennen als Marke eines Kandidaten, als darum, dass die Dipinti den gewohnten Gestaltungsmustern entsprachen. Dass sich diese erst mit der Zeit etablierten, ist an den sogenannten programmata antiquissima zu erkennen, die noch anderen Traditionen verpflichtet waren. Dabei spielt es vielleicht sogar weniger eine Rolle, zu zeigen, dass man sich einen professionellen Schreiber leisten konnte, als dass das Bewusstsein über die Konventionen vorhanden war. Wer sich um ein städtisches Amt bewarb, musste dies offenbar in einem Rahmen tun, der für alle gleichermaßen galt. Das Einhalten und die Reproduktion der Gestaltungsnormen zeigten, dass man zu einem bestimmten Teil der Gesellschaft gehörte, dass man sich einzufügen wusste und dass man sich so verhielt, wie die Mitbewerber und die Wahlberechtigten es erwarteten. ${ }^{402}$ Dass sich solche Normen und unausgesprochenen Regelungen in der frühen Kaiserzeit entwickelten, ist kein Zufall. Anhand der Namen der Kandidaten aber auch an den Grab-, Stifter- und Ehreninschriften ist zu erkennen, dass seit der augusteischen Zeit Personengruppen Zugang zu den öffentlichen Ämtern erhielten, deren Familien zuvor keine Hauptrolle im öffentlichen Leben gespielt hatten. Für solche Kandidaten war es besonders wichtig, zu zeigen, dass sie sich demonstrativ an die Konventionen hielten. ${ }^{403}$

Diese Überlegungen lassen sich mit Sicherheit nicht eins $\mathrm{zu}$ eins auf andere Inschriftengattungen übertragen. Doch gerade bei Inschriften, die ebenfalls inhaltlich in hohem Maße mit gängigen oder sogar notwendigen Textbestandteilen operierten, wie etwa Grabinschriften oder Ehreninschriften, finden sich auch regelmäßig einheitliche Gestaltungsmuster. Diese bringen es einerseits mit sich, dass heute auch stark fragmentierte Inschriften anhand bestimmter Gestaltungsmerkmale und kurzer Textteile einer inhaltlichen Gattung zugewiesen werden können. Dabei wäre zum Beispiel an einen Putzrest zu denken, auf dem ein 40 bis $50 \mathrm{~cm}$ hohes $<\mathrm{I}>\mathrm{zu}$ sehen ist, das mit roter oder schwarzer Farbe in einer Schrift, die zwischen actuaria und monumentalis einzuordnen ist, geschrieben wurde. Bei einem solchen Fund würde einiges dafür sprechen, den Buchstaben als Rest eines edictum muneris zu deuten. ${ }^{404}$ Umgekehrt würde man den gleichen Buchstaben, wenn er mit einem spitzen Gegenstand in Stein oder Putz geritzt wurde, wohl nie als Hinweis auf eine Spielankündigung verstehen.

402 Diese Beobachtungen müssen nicht im Widerspruch zu der bei Macrobius überlieferten Aussage Ciceros stehen, die Franklin an den Beginn seiner Überlegungen zu den Dynamiken der politischen Konkurrenz in Pompeji stellt: nam cum ab hospite suo P. Mallio rogaretur ut decurionatum privigno eius expediret adsistente frequentia dixit: Romae, si vis, habebit; Pompeis difficile est. (Macr. Sat. 2.3.11 nach: Ambrosii Theodosii Macrobii Saturnalia apparatu critico instruxit in somnium Scipionis commentaries selecta varietate lectionis ornavit Iacobus Willis (Leipzig 1963). Vgl. Franklin 2001, 1. Vielmehr stellt sich dabei die Frage, welche Rolle die programmata im Rahmen der ambitio in Pompeji spielten. 403 Vgl. zu den in der julisch-claudischen Zeit zu Einfluss gekommenen Familien: Castrén 1975, 119121; Franklin 2001, 131-153.

404 Vgl. dazu auch die inhaltliche Typisierung der edicta munerum in Sabbatini Tumolesi 1980, 116-119. 
In ähnlicher Weise ist auch bei den Schreibwerkzeugen grundsätzlich zwischen Graffiti auf der einen Seite sowie Dipinti und Steininschriften auf der anderen Seite zu differenzieren. Während bei den Dipinti und bei den Steininschriften sehr spezifische und eng mit der Herstellung solcher Inschriften verbundene Werkzeuge zum Einsatz kamen, konnte ein Graffitischreiber viele verschiedene und oft gerade nicht an den Zweck des Schreibens gebundene Utensilien verwenden. Das Schreiben von Steininschriften und Dipinti war somit eine Praxis, die jeweils auf einen bestimmten Personenkreis mit spezifischen Kenntnissen beschränkt war. Wenn jemand, der keine entsprechende Ausbildung besaß, einen Dipinto oder eine Steininschrift anfertigte, konnte es sein, dass sein Werk unter den vielen professionell geschriebenen Inschriften als nicht konform auffiel. Beim Graffitischreiben spielten solche Faktoren hingegen eine andere Rolle. Es sind unterschiedlichste Schreibgeräte belegt. Gerade auch Kinder und Personen, die etwa als Sklaven oder Prostituierte in anderen Kontexten nicht an öffentlich stattfindender Kommunikation teilnehmen konnten, partizipierten an dieser Praxis. ${ }^{405}$ Damit einher geht die Überlegung, dass Graffiti anders als Steininschriften und Dipinti nicht im Rahmen eines Netzwerkes aus Auftraggebern, Handwerkern und Financiers entstanden, sondern dass hier der Ausführende allein, ohne organisatorischen Vorlauf und meist ohne Autorisierung den Schreibakt in Angriff nahm. ${ }^{406}$ Wer einen Graffito schreiben wollte, musste nicht vorher Farbe angerührt und verschiedene Utensilien wie Pinsel und Leiter vorbereitet und mitgebracht haben. Im Zweifelsfall eignete sich auch ein Metallstück, ein Schlüssel oder eine herumliegende Scherbe, falls man sich entschloss, etwas auf eine Wand zu schreiben oder zu zeichnen. ${ }^{407}$ Die Befunde zeigen in einigen Fällen, dass auch Schreibgeräte, die wie die stili eng mit dem Feld des professionellen Schreibens verknüpft sind, verwendet wurden.

Auch für die Schreiber galt, dass sie einerseits Inschriften schaffen mussten, die den Wünschen der Auftraggeber und in den meisten Fällen wohl den Sehgewohnheiten und Vorgaben von dritter Seite entsprachen. Bei den Dipinti und Steininschriften ist ein hoher Grad an Spezialisierung zu beobachten. Gerade bei den Dipinti ist zu betonen, dass der Vorgang des Malens zwar mit Sicherheit nicht so zeitaufwendig war wie das Meißeln einer Steininschrift. Doch das Schreiben mit dem Pinsel erforderte viel Übung und eine sichere Technik, insbesondere, wenn die Dipinti in einer hochgradig elaborierten actuaria oder beinahe monumentalis ausgeführt werden sollten. ${ }^{408}$ Bei den Graffiti dagegen besteht gerade ein konstitutives Merkmal darin, dass jeder, der Schreibkenntnisse besaß, an dieser Praxis aktiv teilnehmen konnte. Wer keine oder nur geringe Kenntnisse im Schreiben und Lesen besaß, konnte zum Beispiel an

405 Vgl. Huntley 2011, besonders 73-76. 79. 83-87.

406 In diese Richtung geht Solin, wenn er schreibt, dass „,compositore ed esecutore coincidono“, und Graffiti „rivelano il sentimento spontaneo“: Solin 1979, 278. 284. Auch Tanzer 1939, 5.

407 So auch: Bagnall 2011, 10 und Langner 2001, 12-13.

408 Vgl. Catich 1968, 163-169. 
den Stellen, wo andere etwas geschrieben hatten, eine Zeichnung hinzufügen, oder aber vielleicht sogar seine Fertigkeiten trainieren, indem er die Buchstaben nachahmte, worauf manche Teilbefunde hindeuten. ${ }^{409}$

Für die Betrachter in der Antike, die sich nicht mit der Rekonstruktion des Textes der einzelnen Inschrift befassten, sondern solchen Dipinti, Graffiti und Steininschriften täglich in ihrem Umfeld begegneten, spielten diese Schemata ebenfalls eine wichtige Rolle. Sie erlaubten es ihnen, die Inschriften, auch ohne sie im einzelnen zu lesen, anhand des optischen Eindrucks zumindest grob inhaltlich einzuordnen und gegebenenfalls zu beurteilen, ob sie überhaupt von Interesse waren. Auch dies trifft insbesondere auf die Dipinti zu. Bei den Steininschriften waren die Inhalte deutlich stärker mit den Aufstellungs- oder Anbringungsorten der Inschriften korreliert, sodass ein Passant zum Beispiel davon ausgehen konnte, dass die meisten Steininschriften, denen man auf dem forum begegnete, Ehren- oder Stifterinschriften waren. Die Graffiti hingegen waren inhaltlich derart vielfältig, dass gerade bei kürzeren Exemplaren kaum eine Einordnung über das Schriftbild oder ähnliches zu leisten war. ${ }^{410}$ Bei den Dipinti, unter denen vor allem programmata und edicta munerum häufig auf derselben Wand standen, sodass sich ein Passant oder Betrachter manchmal einer zunächst unüberschaubaren Menge an Inschriften zugleich gegenüber sah, konnte es jedoch signifikant sein, dass auch mit einem flüchtigen Blick schon ein Eindruck darüber gewonnen werden konnte, welche Inhalte zu erwarten waren. Wenn ein Betrachter zum Beispiel nach der Wahl an einer Wand vorüberging und des Umstandes gewahr wurde, dass dort nur programmata angebracht waren, würde er diesen vermutlich weniger Aufmerksamkeit geschenkt haben, als wenn ein neuer, als edictum muneris zu erkennender Dipinto hinzugekommen wäre.

\subsection{Die Verteilung der Schriftzeugnisse im Stadtgebiet}

Außer den bereits beschriebenen Aspekten des Inhaltes und der materialen Beschaffenheit ist auch die räumliche Verteilung von erheblicher Bedeutung hinsichtlich der Funktionen von Schrift im öffentlichen Raum. Sie spielt neben den beteiligten Akteuren die größte Rolle, wenn es darum geht, zu klären, in welcher Situation und mit welchen Absichten eine Inschrift angebracht wurde. Zwar kann lediglich eine detaillierte Betrachtung ausgewählter kleinräumiger Ensembles zu tiefgehenden Erkenntnissen hinsichtlich der Situation, des Anlasses und der Rezeptionsbedingungen führen. ${ }^{411}$ Für diese detaillierten Analysen wird im Folgenden als Hintergrund die Verteilung

409 Vgl. zu ungeübten Schreibern: Benefiel 2011, 28-29. Vgl. Langner 2001, 121-122.

410 Dies gilt natürlich nicht für solche Graffiti, deren Inhalt eine sehr spezifische Form mit sich brachte, wie etwa Zahlenreihen, Listen oder längere Epigramme, die sich über mehrere Zeilen verteilten. Vgl. dazu Kruschwitz 2008.

411 Diese wird in mehreren Fallbeispielen in Kapitel 4 durchgeführt. 
der Inschriften im gesamten Stadtgebiet von Pompeji beschrieben. ${ }^{412}$ Da die Dokumentation in Herculaneum zu viele Lücken aufweist, ist eine Kartierung dort derzeit nicht sinnvoll. ${ }^{413}$

Für die drei Gattungen wird in den folgenden Abschnitten gefragt, 1. wo solche Inschriften angebracht wurden, 2. ob Korrelationen zwischen Inhalt und Anbringungsstellen festzustellen sind ${ }^{414}$ und 3. als Zwischenergebnis, ob und wo sich Akkumulationen aller drei Inschriftenarten fanden.

\subsubsection{Stein- und Metallinschriften}

Stein- und Metallinschriften konzentrieren sich in bestimmten Bereichen der Stadt, die man als Pole des öffentlichen Raumes bezeichnen kann: ${ }^{415}$ Auf öffentlichen Platzanlagen, in den Nekropolen und in öffentlichen Gebäuden: in der basilica, im Theater, im Amphitheater oder in Tempeln (Abb. F42). Dies gilt besonders für Grabinschriften, Ehreninschriften an entsprechenden Monumenten und Inschriften, die von Stiftungen und Weihungen ganzer Gebäude oder aber einzelner Elemente und Ausstattungsgegenstände zeugen und in einem besonderen inhaltlichen Bezug zum Umfeld stehen. Hinzu kommen Inschriften, die an bestimmten Punkten im Straßennetz verortet waren, etwa weil sie sich auf ein dort platziertes Monument oder auf ihr Umfeld bezogen.

In Pompeji stellen die Grabinschriften die größte Gruppe an erhaltenen Steininschriften dar. In den dortigen Nekropolen wurden zahlreiche tituli gefunden, die an

412 Neben den erhaltenen Inschriften und den Angaben im CIL sind besonders wichtig: Fiorelli 1875; Außerdem Gell/Gandy 1824; Mazois III 1824 und Mazois II 1824; Vgl. z. B. den Eintrag zu CIL IV 1136. Della Corte bietet manchmal in der Erstveröffentlichung in den Notizie degli Scavi mehr Informationen als im CIL: Della Corte 1911; Della Corte 1914; Della Corte 1936; Della Corte 1946; Della Corte 1958b. 413 Insbesondere bei den Graffiti verzerrt derzeit die Dokumentation den Eindruck vom Befund erheblich. Gerade für die Außenbereiche sind nur sehr wenige Einträge im CIL aufgenommen. Das Herculaneum Graffiti Project unter der Leitung von Rebecca Benefiel konnte dagegen bereits bei der ersten Kampagne zahlreiche bisher unpublizierte Zeichnungen und Texte aufnehmen. Die Publikation der addenda durch Antonio Varone ist in den nächsten Jahren zu erwarten. Zu der Frage, in welchem Zusammenhang die geringe Anzahl der bekannten Graffiti und Dipinti in Herculaneum mit der Überlieferung und Dokumentation steht: Solin 1973b, 100-101; Wallace-Hadrill 2011a, 290-292. Während für die geringe Anzahl an Dipinti sicherlich zurecht unterschiedliche soziale und politische Strukturen verantwortlich gemacht werden, zeigen doch die Neufunde bei den Graffiti, dass hier das Bild zu korrigieren wäre. Die Statuen und Steininschriften sind zwar zahlreich, aber oft ohne eindeutig identifizierbaren Kontext dokumentiert. Vgl. Lahusen/Formigli 2007, 142-151; Wallace-Hadrill 2011c, 128-145; Pagano 2000, 86; Allroggen Bedel 2008, 47-53.

414 Insbesondere diese Frage kann aufgrund der Menge des Materials nicht erschöpfend behandelt werden, spielt aber bei den Fallbeispielen eine wichtige Rolle.

415 In Anlehnung an den Begriff der drei Pole des öffentlichen Raumes in frühen griechischen Städten nach Hölscher 1998, 24. 
den Außenseiten der Monumente angebracht bzw. auf die öffentliche Wahrnehmung hin ausgerichtet waren. ${ }^{416}$ Vor allem entlang den Straßen, die von der Porta Ercolano und der Porta Nocera aus der Stadt hinausführen, wurde jeweils eine große Anzahl an Grabbauten freigelegt, die auch heute noch in situ erhalten sind. Vor den Porte Vesuvio, Nola, Stabia und Marina fanden sich einige weitere Inschriften. ${ }^{417}$ In Herculaneum dagegen wurden die Nekropolen bisher nicht systematisch ergraben, da sie tief unter der modernen Bebauung liegen. Daher sind dort nur vereinzelte Zeugnisse von Bestattungen und Grabinschriften bekannt. Zum einen ist dies das Grabmal des Marcus Nonius Balbus auf der Terrasse oberhalb der antiken Küste. ${ }^{418}$ Außer diesem herausragenden Monument kamen auch bei den Ausgrabungen im 18. Jh. Grabmonumente zutage. ${ }^{419}$ Darüber hinaus konnten in Herculaneum bisher nur wenige und eher bescheidene Spuren von Bestattungen verzeichnet werden, die allerdings topologisch insofern von Bedeutung sind, als durch sie eine Annäherung an die Bestimmung der Stadtgrenzen möglich ist. ${ }^{420}$ Die Stelen in Hermenform, welche sowohl in Herculaneum als auch in Pompeji belegt sind, werden hier ausgeklammert, da sie meist nicht auf den öffentlichen Raum hin ausgerichtet, sondern innerhalb des Grabbezirkes aufgestellt waren und so den Blicken von Passanten verborgen blieben. ${ }^{421}$ Außer den Grabinschriften kommen die cippi des Titus Suedius Clemens hin$z^{4}{ }^{422}$ Ein singuläres Zeugnis stellt eine defixio an der Fassade des Grabes OS 23 in

416 Vgl. ausführlich zu den pompejanischen Grabinschriften, ihre Aussagen und Gestaltung: Campbell 2015, 61-83.

417 Bei einer Ausweitung des Grabungsgeländes in diesen Bereichen wäre mit weiteren Grabmonumenten und daran angebrachten Textträgern zu rechnen. Einige der heute bekannten Gräber sind mittlerweile ihrer Inschriften beraubt. Es ist jedoch anzunehmen, dass im Grunde alle Grabbauten mit einem titulus versehen waren, in dem zumindest der oder die Grabinhaber sowie weitere zur Bestattung zugelassene Personen(gruppen) genannt wurden.

418 Vgl. Guidobaldi 2008, 63-64.

419 In einem davon waren Angehörige oder Freigelassene der Gens Nonia bestattet. Es befindet sich ca. 200 m geographisch südlich des heutigen, am Corso Resina gelegenen Haupteinganges zum Grabungsgelände. Dazu gehören die Inschriften CIL X 1473-1475. 1477. Zur Lokalisierung: Cochin/Bellicard 1755, 24-28 und Taf. 6; Ruggiero 1885, XXXVII-XXXVIII; Maiuri 1958a Taf. 2 im Bereich des mit ,Moscardino‘ markierten Grundstückes. Vgl. Parslow 1995, 335 Anm. 16; 338 Anm. 3. Cormack 2007, 586. Eine weitere Grabinschrift ohne Kontext ist CIL X 1468.

420 Pagano 1989, 271. Die von Pagano genannte Kreuzung Via Tirone di Moccia/Via Marconi ist jedoch in östlicher Richtung recht weit von der Ausgrabungsstätte entfernt. Die Bescheidenheit der Funde spricht zudem dafür, dass es sich nicht um die Bestattung einer hochrangigen Person handelt und somit nicht davon ausgegangen werden kann, dass sich das Grab in nächster Nähe der Stadtgrenze befand.

421 Vgl. dazu: Kockel 1983, 16-18. Die Stelen bestehen aus Kalkstein, Tuff, Basalt oder Marmor. Inschriften finden sich auf ca. einem Drittel der Exemplare und nur auf solchen aus Marmor. Auch wenn diese Stelen bereits früher im 1. Jh. v. Chr. regelmäßig zur Ausstattung von Bestattungsstellen aller Gesellschaftsschichten gehörten, war es anscheinend erst ab augusteischer Zeit üblich, sie zu beschriften: Kockel 1987, 188-189.

422 Siehe unten, Kapitel 4.3.6. 
der Nekropole an der Porta di Nocera dar. ${ }^{423}$ In der Nekropole vor der Porta Ercolano wurde vermutlich zudem eine Art Werbeinschrift für eine Badeanlage am Meer gefunden (CIL X 1063). ${ }^{424}$

Die zweite große Gruppe bilden die Ehreninschriften. In Tabelle 3 sind die pompejanischen Ehreninschriften zusammengestellt, für die das CIL oder der noch erhaltene Befund Angaben zum Aufstellungskontext erlauben. Hinzu kommen, besonders am forum, ca. 60 Sockel, die heute und wohl schon bei der Ausgrabung ihrer Verkleidungen beraubt waren, die jedoch vor dem Vulkanausbruch aller Wahrscheinlichkeit nach sowohl Bildwerke als auch die zugehörigen Inschriften trugen. Vor allem an diesem Platz muss man sich die Präsenz von Schrift daher viel dichter vorstellen, als dies heute zu erkennen ist. ${ }^{425}$ Daraus ergibt sich, dass das forum mit bis zu ca. 70 schrifttragenden Ehrenmonumenten ausgestattet gewesen sein könnte. ${ }^{426}$ Das Theater bildete neben diesem zentralen Platz mit zumindest fünf Denkmälern einen weiteren Schwerpunkt, auch wenn vier davon den Holconii gewidmet waren, die unter anderem am Theater umfangreiche Bauarbeiten sua pecunia hatten durchführen lassen. ${ }^{427}$

Auch Bau- und Stifterinschriften haben sich an einigen größeren öffentlichen Gebäuden erhalten. Wie die Ehreninschriften begegneten sie Passanten vor allem am forum und den umliegenden Gebäuden sowie im Bezirk um die Theater, das Iseum und das Foro Triangolare. Hinzu kommen jedoch eine ganze Reihe von Bauinschriften im Amphitheater, der Porta Stabiana, den Stabianer und den Forumsthermen. ${ }^{428}$ Unter den Bau- und Stifterinschriften sind vier oskische Inschriften, die auch kurz vor dem Vulkanausbruch noch sichtbar waren: die Straßenbau-Inschrift der aídilis ${ }^{429}$ M. Siuttiis und N. Púntiis (Ve 8), die Tafel des Viíbis Aadirans im Iseum (Ve 11) ${ }^{430}$, die Sonnenuhr des Mr. Atiniís in den Stabianer Thermen (Ve 12) und die Inschrift des

423 Vgl. dazu Elefante 1985 und Rodríguez Almeida 2001.

424 Die Fundumstände sind im CIL allerdings unklar beschrieben, da die Inschrift unter den Funden vor der Porta Marina aufgeführt wird, in der Beschreibung jedoch der Eingang der Villa di Cicerone, der an der Straße vor der Porta Ercolano liegt, angegeben wird. Die Tafel befand sich am Fundort zudem wahrscheinlich in Zweitverwendung. Vgl. Cooley/Cooley 2004, 82; Kruschwitz 1999, 247 Abb. 4; $250-251$.

425 Vgl. Kockel 2005, 56 Abb.4; allgemein: Cooley/Cooley 2013, 192; Zu den Statuensockeln in den Vorhallen von macellum und Eumachia-Gebäude, die nach der Ausgrabung massiv restauriert wurden und unter anderem deshalb heute besonders gleichförmig wirken: Wallat 1997, 35f. 157. Zu den Ehrenbögen: Müller/Kockel 2011, 41.

426 Dies gilt, sofern die Sockel vor dem Eumachia-Gebäude und macellum tatsächlich alle bestückt waren.

427 Die Bauinschrift ist in drei Versionen belegt: CIL X 833. 834. 835; Mau 1908, 148-149. Ein weiteres überliefertes Bauprojekt, das Veränderungen am Apollontempel beinhaltete, ist durch die Inschrift CIL X 787 belegt.

428 Vgl. Mau 1908, 503-504.

429 aídilis ist Nom. Pl. von aídil. Vgl. von Planta 1897, 499. 675.

430 Viíbis Aadirans ist zwar der Stifter, doch erbaut wurde das Gebäude, das sich heute im Westen an das Iseum anschließt vom kvaísstur Viínikiís Maraheís. 
Tabelle 3: Ehreninschriften im öffentlichen Raum und in Heiligtümern und dem Theater von Pompeji.

\begin{tabular}{|c|c|c|c|c|}
\hline Kontext & CIL X & Fundstelle & Geehrter & Material \\
\hline \multirow{8}{*}{ Forum } & 788 & $\begin{array}{l}\text { Statuensockel an W-Seite } \\
\text { des Platzes }\end{array}$ & $\begin{array}{l}\text { Marcus Lucretius } \\
\text { Decidianus Rufus }\end{array}$ & Kalkstein \\
\hline & 789 & $\begin{array}{l}\text { Statuensockel an W-Seite } \\
\text { des Platzes }\end{array}$ & $\begin{array}{l}\text { Marcus Lucretius } \\
\text { Decidianus Rufus }\end{array}$ & Marmor \\
\hline & 790 & $\begin{array}{l}\text { Statuensockel an W-Seite } \\
\text { des Platzes }\end{array}$ & $\begin{array}{l}\text { Caius Cuspius Pansa } \\
\text { (Vater) }\end{array}$ & Kalkstein \\
\hline & 791 & $\begin{array}{l}\text { Statuensockel an W-Seite } \\
\text { des Platzes }\end{array}$ & $\begin{array}{l}\text { Caius Cuspius Pansa } \\
\text { (Sohn) }\end{array}$ & Kalkstein \\
\hline & 792 & $\begin{array}{l}\text { Statuensockel an O-Seite } \\
\text { des Platzes, südlich von } \\
\text { Via dell'Abbondanza }\end{array}$ & Quintus Sallustius & $\begin{array}{l}\text { Buntmarmor } \\
\text { (Pavonazzet- } \\
\text { to?) }\end{array}$ \\
\hline & 795 & - & Augustus & Cipollino \\
\hline & 798 & $\begin{array}{l}\text { unterhalb des Bogens am } \\
\text { NO-Zugang des Platzes }\end{array}$ & $\begin{array}{l}\text { Angehöriger des } \\
\text { Kaiserhauses }\end{array}$ & Marmor \\
\hline & 799 & - & Livia & Marmor \\
\hline \multirow{2}{*}{$\begin{array}{l}\text { Gebäude der } \\
\text { Eumachia }\end{array}$} & 813 & $\begin{array}{l}\text { Nische in der östlichen } \\
\text { Krypta }\end{array}$ & Eumachia & Marmor \\
\hline & $814 a$ & - & Caius Norbanus Sorex & Marmor \\
\hline $\begin{array}{l}\text { Tempel der } \\
\text { Fortuna Augusta }\end{array}$ & 823 & in oder bei dem Tempel & Augustus & Marmor \\
\hline $\begin{array}{l}\text { Kreuzung } \\
\text { Via dell'Abb. / } \\
\text { Via Stab. }\end{array}$ & 830 & $\begin{array}{l}\text { Statuensockel, der einem } \\
\text { Ziegelpfeiler vorgelagert } \\
\text { ist }\end{array}$ & $\begin{array}{l}\text { Marcus Holconius } \\
\text { Rufus }\end{array}$ & Marmor \\
\hline $\begin{array}{l}\text { Foro } \\
\text { Triangolare }\end{array}$ & 832 & $\begin{array}{l}\text { Nordende des } \\
\text { Platzes }\end{array}$ & $\begin{array}{l}\text { Marcus Claudius } \\
\text { Marcellus }\end{array}$ & Marmor \\
\hline \multirow{5}{*}{$\begin{array}{l}\text { Theatrum } \\
\text { maius }\end{array}$} & 837 & - & $\begin{array}{l}\text { Marcus Holconius } \\
\text { Rufus }\end{array}$ & Marmor \\
\hline & 838 & in Sitzstufe eingelegt & $\begin{array}{l}\text { Marcus Holconius } \\
\text { Rufus }\end{array}$ & $\begin{array}{l}\text { Marmor / } \\
\text { Bronze }\end{array}$ \\
\hline & 839 & - & $\begin{array}{l}\text { Marcus Holconius } \\
\text { Rufus }\end{array}$ & Marmor \\
\hline & 840 & im Boden der Bühne & $\begin{array}{l}\text { Marcus Holconius } \\
\text { Celer }\end{array}$ & Marmor \\
\hline & 842 & - & Augustus & - \\
\hline Iseum & $814 b$ & südliche porticus & Caius Norbanus Sorex & Marmor \\
\hline
\end{tabular}


Ú. Kampaniis im Boden des Apollontempels (Ve 18). ${ }^{431}$ Unter den lateinischen Bauinschriften waren in mehreren Fällen gleichlautende oder sehr ähnliche Inschriften an verschiedenen Stellen eines Gebäudes angebracht. Besonders bekannt ist das Beispiel des Eumachia-Gebäudes mit der großen Inschrift auf dem Architrav der Vorhalle (CIL X 811) und der deutlich kleinformatigeren Inschrift über dem zweiten Eingang von der Via dell'Abbondanza aus (CIL X 810). Auch die Bauinschrift des Caius Quinctius Valgus und Marcus Porcius am Amphitheater war in doppelter Ausführung an den Zugängen zur arena angebracht, und die Holconii ließen sogar drei fast gleichlautende Inschriften im großen Theater befestigen (CIL X 833-835). Daran zeigt sich deutlich, dass diese Inschriften nicht nur dazu dienten, die finanzielle Aufwendung zu dokumentieren, sondern auch dazu, möglichst vielen Personen, die die Gebäude nutzten, die Leistung vor Augen zu führen.

Bei allen Stein- und Metallinschriften lässt sich ein sehr enger Bezug zwischen dem Text, dem Textträger und den Bildwerken, Gebäuden und Örtlichkeiten, auf die er sich bezieht, feststellen. Die Einwohner oder Gäste Pompejis sahen sich nicht in jeder Straße oder an jeder Kreuzung mit Texten dieser Art konfrontiert, sondern bestimmte Inhalte waren mit spezifischen Orten verknüpft. Dies gilt besonders für Grab- und Ehreninschriften, die entweder zwangsläufig außerhalb der Stadt lagen oder aber als Teil der Monumente erst durch Ratsbeschluss genehmigt werden mussten. Bauinschriften und in besonderem Maße Stifterinschriften, die im Rahmen einer Schenkung einer Einzelperson an das Gemeinwesen stattfanden, ermöglichten es, diesen Rahmen etwas zu erweitern - von den öffentlichen Platzanlagen auch in Gebäude wie die Thermen oder Theater hinein. Dass dabei der Präsenz der Texte, ihrer Sichtbarkeit und der Rezeption durch die Mitbürger ein hoher Stellenwert beigemessen wurde, zeigt sich daran, dass bei manchen Gebäuden gleich mehrere Inschriften, zum Beispiel an den verschiedenen Eingängen, platziert wurden, und dass zwischen der Ausrichtung der Ehreninschrift und des dazu gehörenden Bildwerkes immer enge Beziehungen bestanden. Diese Inschriften bezogen ihre Bedeutung wesentlich aus dem Gegenstand, an dem sie angebracht waren und reicherten diesen im Gegenzug mit Bedeutungen an. Dabei ging es nicht um reine Information, sondern durch die Kennzeichnung wurden die Statuen oder Gebäude erstmals als personenbezogenes Ehrenmonument, als Stiftung oder Weihgeschenk definiert.

431 Außer der Publikation des Textes in Umschrift bei Vetter 1953, siehe zu Ve 8: Abbildung: Guzzo 2007, 110; Fiorelli 1875, 28-29 (im Plan mit d markiert); Zvetaieff 1886 Taf. 10. Zu Ve 11: Sampaolo 1997, 301 Abb. 24-26. Zu Ve 12: Eschebach 1979, 17; 19 Abb. 5; Taf. 51a. Zu Ve 18: Cooley 2002, 79 Abb. 6.1. 


\subsubsection{Dipinti}

Während ein Passant vor allem auf dem forum oder in einigen der öffentlichen Gebäude mit zahlreichen monumentalen, in Stein gemeißelten Texten konfrontiert wurde, waren die rot oder schwarz gemalten Dipinti weder dort noch in der unmittelbaren Umgebung angeschlagen. ${ }^{432}$ Stattdessen häuften sie sich entlang der Straßenzüge. Die Dipinti kamen jedoch nicht an allen Straßenzügen in gleicher Dichte vor, sondern es gab bestimmte Bereiche und Straßen, in denen besonders viele Wandinschriften gemalt worden waren. ${ }^{433}$ Viele Dipinti fanden sich entlang der Via dell'Abbondanza, Via Stabiana, Via delle Terme/Via della Fortuna/Via di Nola sowie Via degli Augustali und Vico dei Vetti. ${ }^{434}$ Bei den ersten drei handelt es sich um die besonders breit angelegten Hauptverkehrsachsen der Stadt. ${ }^{435}$ Die Via degli Augustali und der Vico dei Vetti sind dagegen schmaler. ${ }^{436}$ Dennoch waren beide wichtige Verkehrswege, da der Vico dei Vetti die Via della Fortuna mit der Porta Vesuvio verband und die Via degli Augustali direkt vom forum in den östlichen Teil der Stadt führte. Ein wichtiger Faktor bei der Wahl der Anbringungsstelle war daher mit Sicherheit ein möglichst hoher Publikumsverkehr.

Es waren nicht nur bestimmte Straßenzüge dichter beschrieben als andere, sondern auch innerhalb einer Häuserfront sind jeweils an manchen Fassadenabschnitten regelrechte Dipinto-Cluster zu beobachten. Dies ist deutlich auf den Fotografien bei Stefani und Varone und auf den Aquarellen in Spinazzolas Publikation der Grabungen an der Via dell'Abbondanza zu erkennen (Abb. 31). ${ }^{437}$ Insbesondere in der Umgebung von Eingängen finden sich zahlreiche Dipinti, weniger hingegen an den Ecken der insulae. An den großen, langen Straßen wie der Via dell'Abbondanza ist zudem zu beobachten, dass gegenüber von kleinen Plätzen mit Laufbrunnen oder die Eingänge flankierenden Bänken besonders viele dieser Texte zu lesen oder $\mathrm{zu}$ betrachten waren. Als Beispiele seien die Kreuzungen zwischen Via dell'Abbondanza und Vicolo di Paquius Proculus sowie die gegenüberliegenden Fassaden von III 2, 1 und I 12, 1-3 genannt. ${ }^{438}$

432 Ausnahmen: CIL IV 672-675 und Ve 33 an mehreren Säulen des forum.

433 Zur Kartierung der Dipinti sei auf die Datenbank des Sonderforschungsbereiches 933 Materiale Textkulturen verwiesen: https://pompeji.materiale-textkulturen.de (Stand 1.7.2020).

434 Mouritsen und Laurence kartieren beide die unterschiedliche Dichte mit Hilfe schematischer Markierungen, indem sie die Anzahl der Texte zur Länge der Straßen in Beziehung setzen: Mouritsen 1988, 48 Abb. 3 kartiert nur die programmata, während Laurence „messages“, also sowohl Graffiti als auch Dipinti aufnimmt: Laurence 2007, 109-113. Abb. 6.5-6.8.

435 Via Stabiana: zwischen 6,5 und 8,5 m breit. Via dell'Abbondanza: zwischen 6,7 und 8,5 m breit, direkt westlich der Via Stabiana, wo sich die Via dell'Abbondanza platzartig verbreitert, beträgt die Breite jedoch $14 \mathrm{~m}$. Via di Nola: zwischen 6,4 und 9,2 $\mathrm{m}$ breit.

436 Die Via degli Augustali ist zwischen 4,5 und 4,8 m breit.

437 Viitanen et al. 2013, 68. Spinazzola 1953, z. B. Taf. 9.

438 Vgl. die Fallbeispiele in: 4.1.1. 


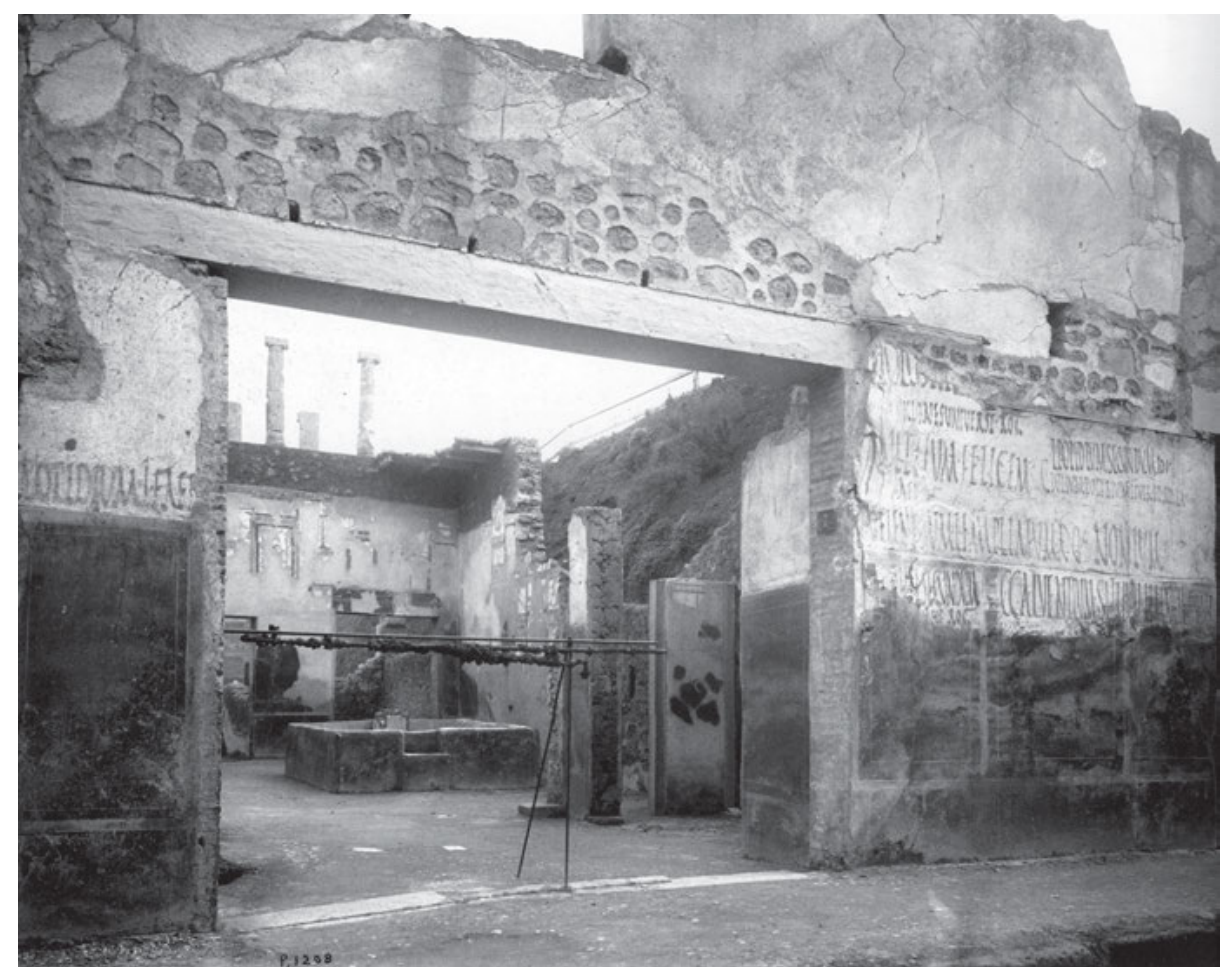

Abb. 31: Fassade der Fullonica di Stefanus I 7, 6 CIL IV 7164-7169. 7171-7173. (C) Su concessione del Ministero per i Beni e le Attività Culturali e per il Turismo - Parco archeologico di Pompei. Jegliche weitere Reproduktion oder Duplikation ist untersagt.

Weitere Bereiche, in denen viele gemalte Inschriften gefunden wurden, sind die Grabbezirke. Aus den Nekropolen vor der Porta Nola und besonders an der Porta Nocera sind zahlreiche Dipinti bekannt. ${ }^{439}$ Auch hier fanden die Schreiber an den hohen Podien oder Umfassungsmauern, die meist mit sehr hellem Stuck verkleidet waren, geeignete Beschreibflächen vor und konnten im Vergleich zu den Hauptstraßen vermutlich relativ ungestört arbeiten.

Die programmata verteilten sich aufgrund ihrer hohen Zahl an den langen Straßenzügen im gesamten Stadtgebiet. Die oft sehr zügige Ausführung und der standardisiete Charakter der Texte führte mit Sicherheit dazu, dass die Schreiber nicht für jeden einzelnen Dipinto nach der optimalen Stelle auf den jeweiligen Wänden suchen konnten. Die Anzahl der edicta munerum ist dagegen deutlich geringer, die Gestaltung aufwendiger und der einzelne Dipinto größer, sodass Aspekte der Verteilung

439 Nekropole Porta di Nola: De Caro 1979, 72. Nekropole Porta Nocera: CIL IV 9936-9961. 99689986. Dies gilt nicht für die Nekropole vor der Porta Ercolano. 
und Positionierung sowie mögliche Gründe dafür klarer hervortreten. ${ }^{440}$ Ein besonders beliebter Ort für deren Anbringung war das nähere Umfeld des Amphitheaters selbst. ${ }^{441}$ Auch entlang der Via dell'Abbondanza waren einige Spielankündigungen zu lesen. Ein weiterer Schwerpunkt ist im Theaterbezirk, in dem auch der ludus gladiatorius liegt, auszumachen. Hinzu kommen weitere Stellen entlang besonders belebter Straßen. Die größte Häufung findet sich jedoch in den Nekropolen an der Porta Ercolano und der Porta Nocera. Da das Amphitheater für die Pompejaner allein mit bis zu ca. 20.000 Plätzen viel zu groß war, ist damit zu rechnen, dass regelmäßig auch Besucher von außerhalb in die Stadt kamen, um die Spiele anzusehen. Diese betraten die Stadt zwangsläufig durch die Nekropolen und insbesondere durch diejenige an der Porta Nocera. Diese war daher der am besten geeignete Ort, um auswärtige Besucher auf bevorstehende Ereignisse hinzuweisen.

Die potentiellen Leser und Passanten sahen sich an einigen Stellen einer schier überwältigenden Menge von Inschriften gegenüber, die geprägt war vom engen räumlichen Nebeneinander inhaltlich wie formal sehr ähnlicher Texte. Dadurch, dass sich die programmata und edicta munerum meist auf ein bestimmtes Ereignis, die nächste Wahl oder Unterhaltungsveranstaltungen beziehen, konnten sie in gewissem Sinne veralten. ${ }^{442}$ Dennoch ließ man sie oft noch Jahre oder Jahrzehnte neben neu entstandenen Inschriften stehen, sodass zu dem räumlichen, inhaltlichen und formalen Nebeneinander eine zeitliche Dimension hinzukommt. Das Verbleichen der Farbe wie auch Übermalungen führten in einigen Fällen dazu, dass die Texte nicht mehr vollständig lesbar waren, und somit einen Aspekt ihrer Textualität verloren. ${ }^{443}$ Andere Aspekte kamen dagegen neu hinzu.

\subsubsection{Graffiti}

Graffiti kommen im Gegensatz zu Steininschriften und Dipinti sowohl im öffentlichen Raum als auch in Innenräumen vor. Auch in der Kartierung der im öffentlichen Raum angebrachten Graffiti im Stadtplan von Pompeji zeigt sich aber, dass lokale Häufungen oder Clusterbildungen an einzelnen Fassadenabschnitten auftraten sowie dass einige Bereiche oder Gebäude ausgespart wurden. ${ }^{444}$ So ist zum Beispiel zu beobachten, dass

440 Diese Eigenschaften und Aspekte werden ausführlich anhand des Fallbeispiels der Fassade der Casa di Trebius Valens (III 2, 1), Abschnitt 4.1.1 thematisiert.

441 Vgl. Abschnitt 4.2.

442 So Eck 1999, 57.

443 Dieser Effekt ist insgesamt nicht sehr stark, wie an den bereits vor Jahrhunderten freigelegten Dipinti zu erkennen ist.

444 Zur Kartierung der Graffiti sei auf die Datenbank des Sonderforschungsbereiches 933 Materiale Textkulturen verwiesen: https://pompeji.materiale-textkulturen.de (Stand 1.7.2020). Entlang der Fassaden gibt es Abschnitte, an denen keine Graffiti eingetragen sind. Dies muss jedoch gerade im Außenbereich nicht bedeuten, dass diese Wände tatsächlich nicht beschrieben waren. Wie die Dipinti 
Graffiti (fast) nicht in Tempeln und eher selten in Bädern vorkommen, wofür allerdings jeweils unterschiedliche Gründe zu suchen sind. Besonders zahlreich sind die Graffiti in Pompeji dagegen im campus (II 7) und im Theaterkorridor (VIII 7, 20), was Langner mit dort stattfindenden Aktivitäten und Spektakeln in Verbindung bringt. ${ }^{445}$

Sowohl im Innen- als auch im Außenbereich wurden Graffiti immer wieder jeweils innerhalb eines engen räumlichen Umfeldes, also zum Beispiel auf einen kurzen Wandabschnitt zwischen zwei Eingängen, geschrieben. Für den Innenbereich scheint zu gelten, dass sich Graffiti vor allem in stark frequentierten Räumen befanden, wo die Bewohner oder Gäste Zeit verbrachten oder wo im Tagesverlauf viele Personen entlang gingen. ${ }^{446}$ Bevorzugt wählte man stark frequentierte Orte, was darauf hinweist, dass die Graffiti von den Autoren durchaus mit der Intention angebracht wurden, dass sie gesehen und gelesen würden. ${ }^{447}$ Es ging also nicht nur um den Vorgang der Produktion, sondern auch die Rezeption wird als Teil der Praxis mit angelegt.

An einigen Stellen ist zu beobachten, dass Graffiti an Stellen hinzugefügt wurden, an denen bereits ähnliche Nachrichten oder Zeichnungen zu betrachten und zu lesen waren. ${ }^{448}$ Dazu gehören zum Beispiel Grüße, Zeichnungen ähnlicher Motive und Modifikationen an bereits Bestehendem, aber auch der Austausch von Beleidigungen. Eindrückliche Beispiele stellen hierfür die Fassade der Casa dei Cei (I 6, 15) mit einer Vielzahl karikaturartiger Zeichnungen menschlicher Köpfe, Kreisornamente und textueller Graffiti (Abb. F43) oder die Gladiatorengraffiti an Grab EN 14 (Abb. F44) in der Nekropole vor der Porta Nocera dar. ${ }^{449}$ Auf diese Weise bildeten sich Cluster über

darauf aufgemalt sind, sind auch die meisten Graffiti in den Wandputz geritzt und gingen so an vielen Stellen bereits kurz nach oder im Zuge der Ausgrabung verloren. Anders als die Dipinti sind sie nicht auf den frühen Fotografien, die bereits kurz nach der Freilegung aufgenommen wurden und für die Dipinti eine wertvolle Informationsquelle darstellen, festgehalten worden. Die meisten Fotos in Varone 2012 stammen aus Fotokampagnen, die Varone selbst seit den 90er Jahren des 20. Jh., als sehr viele Graffiti bereits verloren waren, durchgeführt hat.

445 Langner 2001, 117-120. Oft ist eine Motivation direkt aus dem räumlichen Umfeld zu erschließen. So könnten etwa Namensgraffiti, wenn sie in einem öffentlichen Gebäude an bestimmte Stellen geschrieben sind, als eine Art Markierung gelesen werden, wie es Angelos Chaniotis für Befunde in Aphrodisias annimmt: Chaniotis 2011, 196. 201.

446 Langner 2001, 100. Dies zeigt vor allem auch Benefiel in Benefiel 2010a und Benefiel 2011, 29-32. Lohmann 2018, 128-136. 363-365 und DiBiasie 2015, 311-312.

447 So auch: Bagnall 2011, 24; Langner 2001, 113; Gesemann 1996, 171. Lohmann stelle eine Häufung an Hauptverkehrsachsen und bestimmten öffentlichen Gebäuden fest: Lohmann 2018, 136.

448 Dies hat natürlich Solin längst beobachtet: Solin 1979, 283.

$449 \mathrm{Zu}$ den menschlichen Köpfen an der Casa dei Cei siehe auch: Langner 2001, 112 Abb. 62; Taf. 23, 440-451. Die Köpfe scheinen teilweise von immer demselben Zeichner geschaffen zu sein, oder die stilistischen Ähnlichkeiten beruhen auf dem Versuch, eine bereits vorhandene Zeichnung zu imitieren. In Herculaneum wäre in diesem Zusammenhang zum Beispiel der Befund in einem der cubicula im Obergeschoss der Casa dei Cervi (IV 21) zu nennen, wo sehr viele figürliche Graffiti gefunden wurden: Langner 734. 909. 1108-1110. 1024. 1025.1310.1509-1511. 1595. 1596.2318-2323. Dort spielten besonders Hirsche, die von unterschiedlichen Zeichnern stammen, eine große Rolle, die sich interessanterweise auch in der Skulpturenausstattung im Garten dieses Hauses wiederfinden. Vgl. Maiuri 1958a, 323. 
einen gewissen Zeitraum hinweg. Derartige dicht beschriftete Flächen waren in ihrem Aussehen und ihrer Bedeutung für das Umfeld nicht statisch, sondern veränderten sich durch Beiträge unterschiedlicher Personen mit der Zeit und waren für die Beteiligten auch in einer zeitlichen Dimension erfahrbar, die Claire Taylor mit dem Begriff „temporality“ umschreibt. ${ }^{450}$

Bei einigen Fundstellen ist es jedoch sehr unwahrscheinlich, dass zufällig vorbeikommende Passanten die Graffiti sahen und lasen. Im Tonnengewölbe des Grabes EN 12 in der Nekropole an der Porta Nocera (Abb. F45) zum Beispiel wurden mehrere textuelle und figürliche Graffiti gefunden, die nur zu sehen sind, wenn man sich in das Gewölbe, und damit in den Grabbau hineinbeugt oder darin steht bzw. sitzt (z. B. Abb. F46). Dies legt den Schluss nahe, dass manche Personen gezielt nach einem nicht direkt einsehbaren Ort suchten, um Zeichnungen und Texte anzubringen, oder aber, dass solche Stellen möglicherweise einem kleineren Kreis von Freunden bekannt waren, die dort immer wieder etwas hinzufügten. Ähnliches gilt auch für einige extrem klein geschriebene Graffiti, die nur aus nächster Nähe zu sehen sind. Solche Graffiti richteten sich also nicht an die Öffentlichkeit im Allgemeinen, sondern waren an ganz spezifische Adressaten gerichtet, die sich mit dem nötigen Vorwissen an Ort und Stelle einfanden.

Die Anbringungsstelle erlaubt in einigen Fällen auch Mutmaßungen über die Situation, in der der Schreiber oder Zeichner den Graffito schuf. Besonders anschaulich ist dies bei einigen Graffiti, die oberhalb von gemauerten, die Eingänge flankierenden Bänken angebracht wurden. Dafür finden sich in Herculaneum und Pompeji zahlreiche Beispiele. ${ }^{451}$ Hier liegt es nahe, dass Besucher oder auch Kunden, die dort warteten, oder andere Personen, die auf den Bänken Platz genommen hatten, zum Zeitvertreib etwas zeichneten oder schrieben. ${ }^{452}$

\subsubsection{Zwischenergebnis}

Es gab im antiken Pompeji keinen Bereich des öffentlichen Raumes und der öffentlichen sowie halböffentlichen Gebäude, der frei von Schrift gewesen wäre: Überall war man mit Texten, und somit potentiell auch mit Schreibenden oder Lesenden und Betrachtern konfrontiert oder konnte selbst zu einem solchen werden. Für die verschiedenen Inschriftengattungen konnten unterschiedliche Schwerpunkte

\footnotetext{
450 Taylor 2011, 97. 98: „It is clear then that we should see these sites (and graffiti in general) not as static ,pictures' but as dynamic processes of production, re-production and reception over time [...] These places became, therefore, appropriate locations to leave one's mark in very specific ways and areas in which individuals connected themselves with others.“ Vgl. auch Keegan 2011, 172.

451 Um nur wenige von Fassaden mit entsprechenden Befunden zu nennen: Herculaneum: Casa Sannitica (V 1); Casa dell'Ara Laterizia (III 17). Pompeji: Casa del Menandro (I 10, 4); Casa dei Cei (I 6, 15). 452 Dazu besonders: Hartnett 2008, 95-98.
} 
herausgearbeitet werden. Der Teilbereich, in dem sowohl Stein- und Metallinschriften als auch Dipinti und Graffiti nebeneinander vorkamen, sind die Nekropolen, in denen aus je unterschiedlichen Gründen die Anbringung von Inschriften notwendig oder opportun war.

Innerhalb der einzelnen Teilbereiche war die Präsenz von Schrift nicht statisch. Während Stein- und Metallinschriften schon bei der Errichtung eines Monuments oder Bauwerks als integraler Bestandteil mit angelegt wurden, kamen Dipinti und Graffiti sekundär hinzu. Bei Veränderungen im Umfeld konnte dann unter Umständen auch wieder eine neue Stein- oder Metallinschrift nötig werden. Befunde, die Graffiti an für uns unerwarteten Stellen beinhalten, machen ein weiteres Potential dieser Zeichnungen und Texte deutlich. Indem Grüße oder andere Inhalte in die Wände von Innen- oder Außenräumen eingeritzt wurden, wurden sie auch für andere sichtbar. Jeder, der wollte, konnte sich so in die Kommunikation einschalten, die damit gewissermaßen öffentlich wurde. Das Vorkommen solcher ,Dialoge“ an eher privaten oder auch verborgenen Stellen, führte nicht nur dazu, dass diese Kommunikation öffentlich sichtbar wurde, sondern auch dazu, dass diese Räume durch deren dauerhafte Präsenz ,veröffentlicht‘ wurden. Auch eine Wand, die schwach untergliedert war und nicht mit bestimmten Inhalten verknüpft war, konnte auf diese Weise Anziehungspunkte für öffentlichen Interesse bieten. Je nachdem, wie schwer die Graffiti aufzufinden waren, war diese Form der Kommunikation jedoch nur einem Kreis von Eingeweihten zugänglich.

Als weiterer wichtiger Punkt lässt sich festhalten, dass die Beziehung der Steinund Metallinschriften zu ihrem Kontext primär semantischer Natur ist. Die Texte treten in eine inhaltliche Beziehung zum Textträger, ergänzen diesen oder konstituieren einen Teil der Bedeutung. Bei Dipinti und Graffiti hingegen ist die semantische Beziehung zum Kontext in den meisten Fällen schwach ausgeprägt. Stattdessen stehen Praktiken der Produktion und Rezeption im Vordergrund, die für die Wahl der Anbringungsstelle und so für die Verteilung der Inschriften im Stadtgebiet relevant waren: Welche Leserkreise sollten erreicht werden? Wie konnte eine bestimmte Adressatengruppe angesprochen werden? Wo war erfahrungsgemäß mit vielen Lesern zu rechnen? Wo hielten sich die Schreiber gerne auf? Wo konnten sie ungestört (und ohne selbst zum Hindernis zu werden) ans Werk gehen? Wo boten sich geeignete Beschreibflächen? Gab es rechtliche Einschränkungen für Teilbereiche des öffentlichen Raumes? Die genannten Fragen werden im Rahmen der Kontextuntersuchungen in Kapitel 4 ausführlich thematisiert.

Schließlich fand durch die Beschriftung auch eine Aneignung der Wand durch die Schreiber und Auftraggeber statt. Indem eine Steininschrift einen bestimmten Raum beanspruchte, wurde der öffentliche Raum entsprechend markiert und der Name sowie das Ansehen des Stifters oder des Empfängers einer Ehrung entsprechend konnotiert und aufgewertet. Diese Form der Nutzung des öffentlichen Raumes für Inschriften stellte letztlich aber eine Reproduktion sozialer Strukturen dar, die auf diese Weise eine Bestätigung und Aktualisierung erfuhren. So konnten auch so beson- 
dere Orte wie das Iseum von einer Familie vereinnahmt werden. Auch bei den Dipinti wird deutlich, dass es mit den großformatigen und repetitiven Anbrigungen darauf ankam, Präsenz zu erzeugen und zugleich Territorien abzudecken. Die häufig übertriebenen Größen der programmata und edicta munerum standen nicht im Dienste der besseren Lesbarkeit, sondern müssen als materialisierter Bedeutungsanspruch verstanden werden. Dass sie sich nicht am forum fanden, kann kein Zufall sein, sondern hängt mit Sicherheit mit Regularien zusammen, die diesen Platz vor einer solchen Aneignung und Beanspruchung durch Partikularinteressen bewahrten. Und auch bei den Graffiti, die eben viel unauffälliger waren als Dipinti und Steininschriften aber ein breites Themenspektrum abdeckten, standen neben den kommunikativen Funktionen für die Urheber mit Sicherheit auch die Aspekte der Präsenz und überhaupt der öffentlichen Wahrnehmbarkeit der eigenen Person im Vordergrund. Mit Hilfe eines Graffito konnte jeder im öffentlichen Raum sichtbar werden und bestimmte Themen dort ganz konkret räumlich verorten oder ein bescheidenes Werk wie etwa eine Zeichnung oder eine Schreibübung hinterlassen. 
